\title{
General Survey of Polarization Observables in Deuteron Electrodisintegration
}

\author{
Hartmuth Arenhövel ${ }^{1}$, Winfried Leidemann ${ }^{2}$, and Edward L. Tomusiak ${ }^{3}$ \\ ${ }^{1}$ Institut für Kernphysik, Johannes Gutenberg-Universität, D-55099 Mainz, Germany \\ ${ }^{2}$ Dipartimento di Fisica, Università di Trento, and Istituto Nazionale \\ di Fisica Nucleare, Gruppo collegato di Trento, I-38050 Povo, Italy \\ ${ }^{3}$ Department of Physics and Astronomy, University of Victoria, Victoria, BC V8P 1A1, Canada
}

(Dated: December 22, 2018)

\begin{abstract}
Polarization observables in inclusive and exclusive electrodisintegration of the deuteron using a polarized beam and an oriented target are systematically surveyed using the standard nonrelativistic framework of nuclear theory but with leading order relativistic contributions included. The structure functions and the asymmetries corresponding to the various nucleon polarization components are studied in a variety of kinematic regions with respect to their sensitivity to realistic $N N$-potential models, to subnuclear degrees of freedom in terms of meson exchange currents, isobar configurations and to relativistic effects in different kinematical regions, serving as a benchmark for a test of present standard nuclear theory with effective degrees of freedom.
\end{abstract}

PACS numbers: $21.40 .+\mathrm{d}, 24.70 .+\mathrm{s}, 25.30 . \mathrm{Fj}, 13.40 . \mathrm{Fn}$

\section{INTRODUCTION}

Over the past decade we have made a systematic study of inclusive and exclusive deuteron electrodisintegration with special emphasis on polarization observables [1-6]. The main purpose of this study was to reveal to what extent the use of polarized electrons, polarized targets and polarization analysis of the outgoing nucleons will allow a more thorough and more detailed investigation of the dynamical features of the two-nucleon system than is possible without the use of polarization degrees of freedom (d.o.f.). Specifically, our interest was focused on the role of the $N N$-interaction model, of subnuclear degrees of freedom in terms of meson and isobar d.o.f. and in some cases on the role of relativistic effects.

In the first paper of this investigation [1] we have considered the inclusive process, followed in [2] by the exclusive case, $\vec{d}\left(\vec{e}, e^{\prime} N\right) N$, including beam and target polarization but without analysis of the outgoing nucleon polarization. In parallel, as an extension to previous work in photodisintegration $[7,8]$, we have formally derived in [3] all possible polarization structure functions, in total 648 , and linear relations between them since only 324 can be linearly independent considering the fact that each structure function is a hermitean form of 18 independent complex $t$-matrix elements, provided parity conservation holds. Formal expressions for polarization observables, using a different representation scheme for the structure functions, have been given by DMITRASINOviC and Gross [9] where also the question of necessary and sufficient measurements for a complete determination of all transition amplitudes has been discussed in detail. In [4] we have continued our own study by looking at the polarization of one or both of the final state nucleons in the exclusive processes $d\left(\vec{e}, e^{\prime} N\right) N$ and $d\left(\vec{e}, e^{\prime} N N\right)$ with various combinations of beam and target polarizations. Although one has 324 linearly independent observables, they are not independent in the more general sense of considering them as functions of the complex $t$-matrix elements. In view of the fact that for the 18 complex matrix elements one phase can be arbitrarily chosen and thus all observables are functions of 35 independent and real variables, it is obvious that the maximal number of independent observables is 35 . Indeed, there exist quadratic relations among them, reducing the 324 linearly independent observables to the required number. The remaining question then is, which one of the many possible subsets of 35 observables constitutes an independent set. This question has been investigated recently in [5] for a two-body reaction of the type $a+b \rightarrow c+d$, where we have derived a general criterion for the selection of a complete set of independent observables, which subsequently has been applied in [6] to the electromagnetic deuteron break-up. In the latter work we have also derived the relations between our structure functions and the ones of [9]. Most recently, we have obtained the general multipole expansion of the structure functions in [10].

Besides this interest in the hadron dynamics, there is a second aspect which underlines the important role of the deuteron from a different perspective, namely in providing an effective neutron target because of its extremely weak binding. In fact, quasifree reactions on the deuteron are frequently used in order to investigate properties of the neutron. A prominent example is the current interest in the determination of the electric form factor of the neutron $G_{E n}$ in quasifree electron scattering off the deuteron using longitudinally polarized electrons and either a vector polarized target or measuring the polarization of the outgoing neutron. Therefore, we also have studied in detail the sensitivity of polarization observables to $G_{E n}$. 


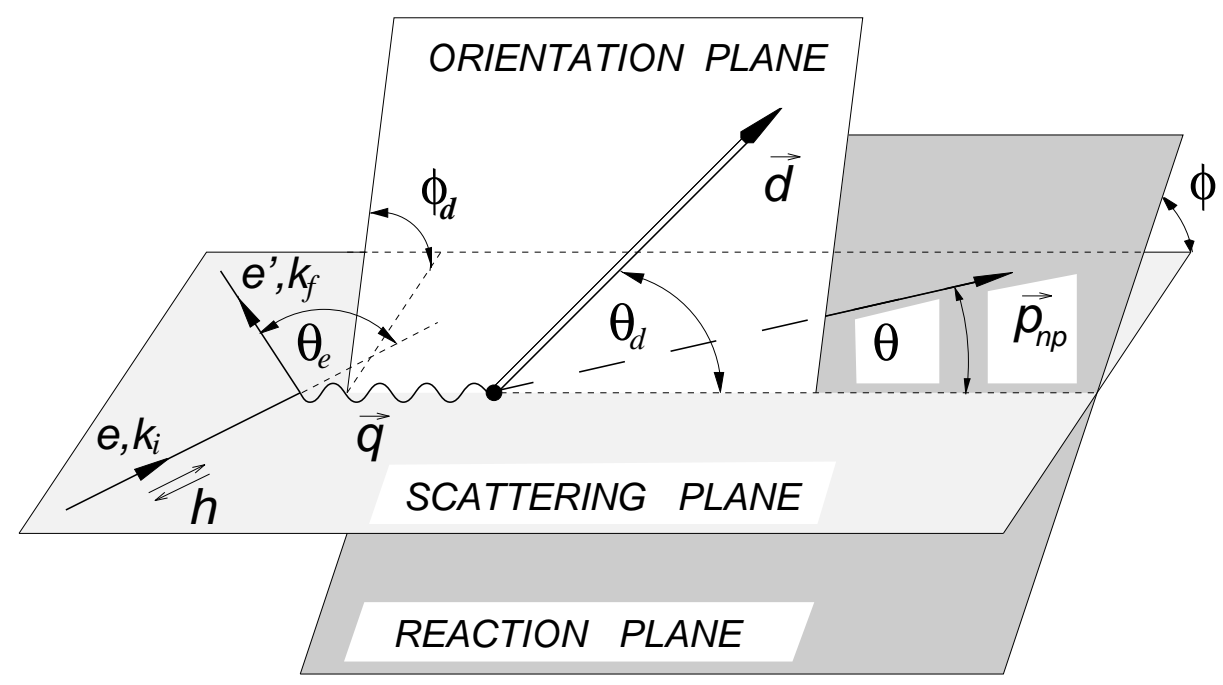

FIG. 1: Geometry of exclusive electron-deuteron scattering with polarized electrons and an oriented deuteron target. The relative $n p$-momentum, denoted by $\vec{p}_{n p}$, is characterized by angles $\theta=\theta_{n p}$ and $\phi=\phi_{n p}$ where the deuteron orientation axis, denoted by $\vec{d}$, is specified by angles $\theta_{d}$ and $\phi_{d}$.

The special and very fundamental role of the two-nucleon system for the investigation of the hadronic structure of nuclei, playing the same role as the hydrogen atom in atomic physics, is underlined first of all by the fact, that $N N$-scattering is of crucial importance for fitting realistic $N N$-potential models. Secondly, the deuteron constitutes the simplest nucleus. It is very weakly bound and allows an exact theoretical treatment, at least in the nonrelativistic regime. Thus it should be clear that such an extensive survey is justified. Moreover, the electromagnetic probe allows a particularly clean and simple interpretation of the associated observables, because it possesses a well known but weak interaction so that in most cases lowest order approaches are sufficient.

With the present work we want to give a concise and self-contained summary of this extensive study in order to provide the interested experimentalist and theorist a sort of handbook for the powerful tool of polarization observables in this fundamental process. We furthermore want to update our previous results with respect to (i) the recent high precision $N N$-potentials, (ii) consistent $\pi$ - and $\rho$-like meson exchange currents, and (iii) complete and consistent inclusion of leading order relativistic contributions in a $v / c$-expansion. Thus we consider this work also as a benchmark for the status of present standard nuclear theory with effective degrees of freedom in terms of nucleon, meson and isobar d.o.f. To this end we will collect in the next section all material relevant for the formal aspects of this process including kinematic properties and relations, and definition of observables for the inclusive as well as for the exclusive reaction in terms of form factors and structure functions, respectively. In Section III we will discuss the experimental separation of structure functions and the question of how to select a complete set of observables. Some calculational details will be presented in Section IV with respect to the hadronic interaction and the electromagnetic current. The latter comprises contributions from one-body and meson exchange currents (MEC) and isobar configurations (IC) as well as leading order relativistic contributions (RC). Then we will discuss in Section V the influence of the above mentioned dynamical effects on various inclusive and exclusive observables for different kinematic regions of energy and momentum transfers. Finally, we will close with a summary and an outlook.

\section{GENERAL FORMALISM}

\section{A. Kinematics}

At first we will consider the kinematic properties of the disintegration process $e+d \rightarrow e^{\prime}+n+p$. The kinematics is governed by the four momenta of the participating particles, i.e., the four-momenta $k_{1}$ and $k_{2}$ of incoming and scattered electron, respectively, and the four-momenta $p_{d}, p_{p}$ and $p_{n}$ of deuteron and outgoing proton and neutron, respectively. In view of on-shell conditions for the participating particles, four-momentum conservation, freedom in the choice of the incoming electron direction and in the choice of orientation of the scattering plane, one is left with five independent variables for this process. Different choices are possible and will be specified below.

The scattering geometry is illustrated in Fig. 1. We distinguish three different planes which all intersect in one line 
as defined by the direction of the three-momentum transfer $\vec{q}=\vec{k}_{1}-\vec{k}_{2}$. First, there is the scattering plane which is defined by the three-momenta of incoming and scattered electrons $\vec{k}_{1}$ and $\vec{k}_{2}$, respectively, then the reaction plane defined by the momentum transfer and the relative momentum $\vec{p}_{n p}=\left(\vec{p}_{p}-\vec{p}_{n}\right) / 2$ of the two outgoing nucleons, and finally the orientation plane as defined again by the momentum transfer and the axis of orientation for a polarized deuteron.

The principal frames of reference are associated with the scattering plane, namely the laboratory frame, and the c.m. frame of the final two nucleons, which is related to the former one by a boost along $\vec{q}$. The $z$-axis is chosen in both frames along $\vec{q}$ and the $y$-axis in the direction of $\vec{k}_{1} \times \vec{k}_{2}$, i.e., perpendicular to the scattering plane. Finally, the $x$-axis is defined by $\vec{e}_{x}=\vec{e}_{y} \times \vec{e}_{z}$ in order to form a right-handed system. The laboratory frame is the natural choice for the experimental determination of observables, whereas the c.m. frame is very convenient for the theoretical calculation. Where necessary, we will indicate by a superscript "lab" or "c.m." to which frame a given quantity refers. With respect to the c.m. frame, we will denote throughout this paper by $\theta$ and $\phi$ the spherical angles of the relative momentum $\vec{p}_{n p}=\left(p^{\text {c.m. }}, \theta, \phi\right)$. Thus the spherical angles of proton and neutron momenta in this frame are $\theta_{p}^{\text {c.m. }}=\theta$, $\phi_{p}^{\text {c.m. }}=\phi$ and $\theta_{n}^{\text {c.m. }}=\pi-\theta, \phi_{n}^{\text {c.m. }}=\phi+\pi$. The final hadronic state is furthermore characterized by the excitation energy $E_{n p}$ which is related to its invariant mass $W_{n p}$ by

$$
E_{n p}=W_{n p}-2 M,
$$

where $M$ denotes the average nucleon mass. Finally, $\theta_{d}$ and $\phi_{d}$ denote the lab frame spherical angles of the deuteron orientation axis in case a polarized deuteron target is employed.

The relevant quantities in the lab frame are the four-momenta of the incoming and scattered electrons $k_{1}^{\text {lab }}=$ $\left(E_{1}^{\text {lab }}, \vec{k}_{1}^{\text {lab }}\right)$ and $k_{2}^{\text {lab }}=\left(E_{2}^{\text {lab }}, \vec{k}_{2}^{\text {lab }}\right)$, respectively, the scattering angle $\theta_{e}^{\text {lab }}$, and the proton and neutron three-momenta $\vec{p}_{p}^{l a b}=\left(p_{p}^{\text {lab }}, \theta_{p}^{\text {lab }}, \phi_{p}^{\text {lab }}\right)$ and $\vec{p}_{n}^{l a b}=\left(p_{n}^{\text {lab }}, \theta_{n}^{\text {lab }}, \phi_{n}^{\text {lab }}\right)$, respectively. For the five independent variables one convenient choice is $E_{1}^{\text {lab }}, E_{2}^{\text {lab }}, \theta_{e}^{\text {lab }}, \theta_{p}^{\text {lab }}$, and $\phi_{p}^{\text {lab }}$. All other quantities are then determined by them. An alternative choice is $E_{1}^{\text {lab }}, E_{2}^{\text {lab }}, \theta_{e}^{\text {lab }}$, and the spherical angles of the relative $n p$-momentum in the c.m. system, $\theta$, and $\phi$. Still another useful choice is the $n p$-final state excitation energy $E_{n p}$, the three momentum transfer $q^{\text {c.m. }}$, again the angles $\theta$ and $\phi$, all with respect to the c.m. frame of the final $n p$-state, and the lab electron scattering angle $\theta_{e}^{\text {lab }}$. This choice is particularly useful for formal investigations of the structure functions, because the latter depend solely on $E_{n p}, q^{\text {c.m. }}$, and $\theta$, if calculated in the c.m. frame.

We will now list the various relevant kinematic quantities in the lab and c.m. frames and their relation to the chosen independent variables. Throughout this work we will denote the square of a four-vector $x_{\nu}$ by $x_{\nu}^{2}=x_{0}^{2}-\vec{x}^{2}$ and use $x=|\vec{x}|$. For given electron momenta $\vec{k}_{1}^{\text {lab }}, \vec{k}_{2}^{\text {lab }}$ and scattering angle $\theta_{e}^{\text {lab }}$, one has for the energy and momentum transfers in the lab frame

$$
\begin{aligned}
& \omega^{\mathrm{lab}}=E_{1}^{\mathrm{lab}}-E_{2}^{\mathrm{lab}}, \\
& \vec{q}^{\mathrm{lab}}=\vec{k}_{1}^{\mathrm{lab}}-\vec{k}_{2}^{\mathrm{lab}},
\end{aligned}
$$

and from this the invariant mass $W_{n p}$ of the $n p$-final state in terms of lab and c.m. frame quantities

$$
\begin{aligned}
W_{n p} & =\sqrt{\left(E_{n p}^{\mathrm{lab}}\right)^{2}-\left(q^{\mathrm{lab}}\right)^{2}} \\
& =\sqrt{\left(M_{d}\left(M_{d}+2 \omega^{\mathrm{lab}}\right)+q_{\nu}^{2}\right)} \\
& =\omega^{\text {c.m. }}+E_{d}^{\text {c.m. }},
\end{aligned}
$$

where

$$
E_{d}^{\text {c.m. }}=\sqrt{M_{d}^{2}+\left(q^{\text {c.m. }}\right)^{2}}
$$

denotes the deuteron c.m. energy, and

$$
E_{n p}^{\mathrm{lab}}=\omega^{\mathrm{lab}}+M_{d}
$$

the lab energy of the final hadronic state. At the photon point $\omega^{\text {lab }}=q^{\text {c.m. }}$ one finds according to (4c) and (5) the relation

$$
\begin{aligned}
q^{\text {c.m. }} & =\frac{W_{n p}^{2}-M_{d}^{2}}{2 W_{n p}} \\
& =\left(E_{n p}+\varepsilon_{d}\right)\left(1-\frac{E_{n p}+\varepsilon_{d}}{2\left(E_{n p}+2 M\right)}\right)
\end{aligned}
$$


where $\varepsilon_{d}$ denotes the deuteron binding energy.

The boost parameter $\gamma$, which governs the transformation from the lab to the c.m. frame and vice versa, is given by

$$
\begin{aligned}
\gamma & =\frac{E_{n p}^{\mathrm{lab}}}{W_{n p}} \\
& =\frac{E_{d}^{\mathrm{c} . \mathrm{m}}}{M_{d}}
\end{aligned}
$$

Energy and momentum transfers in the lab and c.m. frames are related to each other by

$$
\begin{aligned}
\omega^{\mathrm{c} . \mathrm{m} .} & =\frac{1}{W_{n p}}\left(M_{d} \omega^{\mathrm{lab}}+q_{\nu}^{2}\right), \\
q^{\mathrm{c} . \mathrm{m} .} & =\frac{M_{d}}{W_{n p}} q^{\mathrm{lab}} .
\end{aligned}
$$

Similarly, one has for the relative $n p$-momentum

$$
\begin{aligned}
p_{n p}^{\mathrm{lab}} & =p^{\text {c.m. }} \sqrt{1+\left(\frac{q^{\mathrm{c} . \mathrm{m} .}}{M_{d}}\right)^{2} \cos ^{2} \theta}, \\
\cos \theta_{n p}^{\mathrm{lab}} & =\frac{E_{d}^{\mathrm{c} . \mathrm{m} .}}{\sqrt{M_{d}^{2}+\left(q^{\mathrm{c} . \mathrm{m} \cdot}\right)^{2} \cos ^{2} \theta}} \cos \theta, \\
p^{\text {c.m. }} & =p_{n p}^{\mathrm{lab}} \sqrt{1+\left(\frac{q^{\mathrm{lab}}}{W_{n p}}\right)^{2} \cos ^{2} \theta_{n p}^{\mathrm{lab}}}, \\
\cos \theta & =\frac{E_{n p}^{\mathrm{lab}}}{\sqrt{W_{n p}^{2}+\left(q^{\mathrm{lab}}\right)^{2} \cos ^{2} \theta_{n p}^{\mathrm{lab}}}} \cos \theta_{n p}^{\mathrm{lab}} .
\end{aligned}
$$

In the c.m. frame, the nucleon energies are (neglecting the small proton-neutron mass difference)

$$
E_{p}^{\text {c.m. }}=E_{n}^{\text {c.m. }}=E^{\text {c.m. }}=\frac{W_{n p}}{2}=\sqrt{M^{2}+\left(p^{\text {c.m. }}\right)^{2}},
$$

and their three-momenta are given by the relative $n p$-momentum

$$
\vec{p}_{p}^{c . m .}=\left(p^{\text {c.m. }}, \theta, \phi\right), \quad \vec{p}_{n}^{c . m .}=\left(p^{\text {c.m. }}, \pi-\theta, \phi+\pi\right) .
$$

The same quantities in the lab frame may be expressed in terms of the c.m. variables by

$$
\begin{aligned}
E_{p / n}^{\mathrm{lab}} & =\frac{E_{d}^{c . m}}{M_{d}}\left(\frac{W_{n p}}{2} \pm \frac{q^{\text {c.m. }} p^{\text {c.m. }} \cdot \cos \theta}{E_{d}^{c . m}}\right), \\
\left(p_{p / n}^{\text {lab }}\right)^{2} & =\left(\frac{E_{d}^{c . m} p^{\text {c.m. }}}{M_{d}}\right)^{2}+\frac{1}{M_{d}^{2}}\left(\left(q^{\text {c.m. }}\right)^{2}\left[M^{2}+\left(p^{\text {c.m. }}\right)^{2} \cos ^{2} \theta\right] \pm E_{d}^{c . m} W_{n p} q^{\text {c.m. }} p^{\text {c.m. }} \cos \theta\right) .
\end{aligned}
$$

The Jacobian for the transformation $\Omega_{n p}^{\text {c.m. }} \rightarrow \Omega_{i}^{\text {lab }}(i \in\{n, p\})$ is given by

$$
\frac{\partial \Omega_{n p}^{\text {c.m. }}}{\partial \Omega_{i}^{\text {lab }}}=\frac{1}{\gamma}\left(\frac{\beta_{i}^{\text {lab }} \gamma_{i}^{\text {lab }}}{\beta^{\text {c.m. } . ~} \gamma^{\text {c.m. }}}\right)^{3}\left(1+\frac{\beta}{\beta^{\text {c.m. }}} \cos \theta_{i}^{\text {c.m. }}\right)^{-1},
$$

where the angle $\theta_{i}^{\text {c.m. }}$ or $\theta_{i}^{\text {lab }}$ denotes the angle of the momentum of the particle " $i$ " in the indicated frames of reference, and $\beta=\sqrt{\gamma^{2}-1} / \gamma$. Furthermore,

$$
\gamma^{\text {c.m. }}=\frac{E^{\text {c.m. }}}{M},
$$

is the boost parameter that takes particle " $i$ " from its rest system to the c.m. frame. It is the same for both particles. Similarly, for the boost from the particle rest frame to the lab one has

$$
\gamma_{i}^{\text {lab }}=\gamma \gamma^{\text {c.m. }}\left(1+\beta \beta^{\text {c.m. }} \cos \theta_{i}^{\text {c.m. }}\right) .
$$


Note the relations $\theta_{p}^{\text {c.m. }}=\theta$, and $\theta_{n}^{\text {c.m. }}=\pi-\theta$ (see Fig. 1). Furthermore, the particle lab angle is given by

$$
\theta_{i}^{\mathrm{lab}}=\arcsin \left[\frac{\beta^{\mathrm{c} . \mathrm{m} \cdot} \gamma^{\mathrm{c} . \mathrm{m} .}}{\beta_{i}^{\mathrm{lab}} \gamma_{i}^{\mathrm{lab}}} \sin \theta_{i}^{\mathrm{c} . \mathrm{m} .}\right] .
$$

For the description of the polarization components of the outgoing particle, one associates with each particle " $i$ " a frame of reference according to the Madison convention, for which the $z$-axis is taken along the particle momentum, i.e., in the reaction plane, the $y$-axis along $\vec{q} \times \vec{p}_{i}$, i.e., perpendicular to the reaction plane, and the $x$-axis is then determined by the requirement to form a right-handed system. Often the polarization components are evaluated in the c.m. system whereas the experimental measurement is done in the lab frame. Then it is necessary to convert these observables to the laboratory system. Applying nonrelativistic kinematics, the spin eigenstates in either system are simply related by a rotation, $\theta_{i}^{\text {c.m. }}-\theta_{i}^{\text {lab }}$ about the $y$-axis. However, it is well known (cf. refs. [11-13], for example) that for relativistic kinematics there is a correction such that the actual angle of rotation, the Thomas-Wigner angle $\theta_{i}^{W}$, is given by

$$
\theta_{i}^{W}=\arcsin \left[\frac{1+\gamma}{\gamma^{\mathrm{c} . \mathrm{m} .}+\gamma_{i}^{\mathrm{lab}}} \sin \left(\theta_{i}^{\mathrm{c} . \mathrm{m} .}-\theta_{i}^{\mathrm{lab}}\right)\right] .
$$

One readily observes that for nonrelativistic boosts the Wigner angle becomes simply the $\theta_{i}^{\text {c.m. }}-\theta_{i}^{\text {lab }}$. Since the rotation is about the $y$-axis, the $y$-components of the polarization of the outgoing nucleons undergo no change while the $x$ - and $z$-components mix according to

$$
P_{k}^{\mathrm{lab}}(i)=R_{k l}^{W}(i) P_{l}^{\mathrm{c} . \mathrm{m} .}, \quad i \in\{p, n\},
$$

where

$$
R^{W}(i)=\left(\begin{array}{ccc}
\cos \theta_{i}^{W} & 0 & \sin \theta_{i}^{W} \\
0 & 1 & 0 \\
-\sin \theta_{i}^{W} & 0 & \cos \theta_{i}^{W}
\end{array}\right) .
$$

Similarly, double polarization observables transform as

$$
P_{k l}^{\mathrm{lab}}=R_{k k^{\prime}}^{W}(p) R_{l l^{\prime}}^{W}(n) P_{k^{\prime} l^{\prime}}^{\mathrm{c} \cdot \mathrm{m} .} .
$$

\section{B. Definition of observables}

The most general form of an observable in deuteron electrodisintegration is

$$
\begin{aligned}
\mathcal{O}\left(\Omega_{X}\right) & =P_{X} S_{0} \\
& =\operatorname{tr}\left(\mathcal{T}^{\dagger} \Omega_{X} \mathcal{T} \rho_{i}\right),
\end{aligned}
$$

where

$$
S_{0}=\frac{d^{3} \sigma_{0}}{d k_{2}^{\text {lab }} d \Omega_{e}^{\text {lab }} d \Omega_{n p}^{\text {c.m. }}}
$$

denotes the unpolarized cross section. $\Omega_{X}$ is an operator in the final two-nucleon spin space with $P_{X}$ as corresponding polarization observable. Its specific form depends on the analysis of the hadronic final state, i.e., whether or not polarization components of one or both outgoing nucleons are measured, and is defined below. Polarization analysis of the scattered electron is not considered here. $\mathcal{T}$ denotes the reaction matrix, and $\rho_{i}$ the density matrix for the spin degrees of the initial system. The trace refers to all initial state spin degrees of freedom comprising incoming electron and target deuteron.

In the one-photon-exchange approximation the reaction matrix $\mathcal{T}$ separates into a leptonic and a hadronic part, and one obtains from (22) the well-known expression

$$
\mathcal{O}\left(\Omega_{X}\right)=3 c\left(k_{1}^{\mathrm{lab}}, k_{2}^{\mathrm{lab}}\right) \operatorname{tr}\left(T^{\dagger} \Omega_{X} T \rho_{i}\right),
$$

where the hadronic part is represented by the $T$-matrix which is related to the current matrix element between the initial deuteron state and the final $n p$-scattering state. The electron kinematics refers to the lab frame while the $T$ matrix and all quantities of the final $n p$-state refer according to our choice to the final state c.m. system. In (24) the 
initial state density matrix $\rho_{i}$ refers now to the spin degrees of the exchanged virtual photon and the deuteron, i.e. the virtual photon polarizations $\lambda(=0, \pm 1)$ and the deuteron spin projections $\lambda_{d}$ with respect to a chosen quantization axis, here parallel to $\vec{q}$. Furthermore, the kinematic factor in (24) is

$$
c\left(k_{1}^{\mathrm{lab}}, k_{2}^{\mathrm{lab}}\right)=\frac{\alpha}{6 \pi^{2}} \frac{k_{2}^{\mathrm{lab}}}{k_{1}^{\mathrm{lab}} Q^{4}},
$$

with $\alpha$ denoting the fine structure constant and $Q^{2}=-q_{\nu}^{2}$ the four-momentum transfer squared $\left(q=k_{1}-k_{2}\right)$. This factor is related to the Mott cross section $\sigma_{\text {Mott }}$ by

$$
c\left(k_{1}^{\text {lab }}, k_{2}^{\text {lab }}\right)=\frac{1}{6 \pi^{2} \alpha} \frac{\tan ^{2}\left(\theta_{e}^{\text {lab }} / 2\right)}{Q^{2}} \sigma_{\text {Mott }}, \quad \text { where } \quad \sigma_{\text {Mott }}=\frac{\alpha^{2}}{4\left(E_{1}^{\text {lab }}\right)^{2}} \frac{\cos ^{2}\left(\theta_{e}^{\text {lab }} / 2\right)}{\sin ^{4}\left(\theta_{e}^{\text {lab }} / 2\right)} .
$$

The explicit form of the unpolarized cross section in terms of structure functions is given below in (53). The spin degrees of the final state may be taken as $s$, the total spin of the $n p$-final state, and $m_{s}$ its projection on the relative $n p$-momentum $\vec{p}_{n p}$ in the final $n p$-c.m. system. Another convenient choice are the helicities $\lambda_{p}$ and $\lambda_{n}$ of proton and neutron, respectively.

Then the $T$-matrix of (24) between the initial deuteron state $\left|\lambda_{d}\right\rangle$ and the final $n p$-scattering state $\left|m_{1} m_{2}\right\rangle$, both in non-covariant normalization, is given by

$$
\begin{aligned}
T_{m_{1} m_{2} \lambda \lambda_{d}}(\theta, \phi) & =-\pi \sqrt{2 \alpha p_{n p} E^{\mathrm{c} \cdot \mathrm{m} \cdot E_{d}^{\mathrm{c} \cdot \mathrm{m} \cdot} / M_{d}}}\left\langle m_{1} m_{2}\left|J_{\lambda}(\vec{q})\right| \lambda_{d}\right\rangle \\
& =e^{i\left(\lambda+\lambda_{d}\right) \phi} t_{m_{1} m_{2} \lambda \lambda_{d}}(\theta),
\end{aligned}
$$

where $\lambda=0, \pm 1$, and the spherical angles of the relative momentum $\vec{p}_{n p}$ of the final neutron-proton state in the c.m. system are denoted by $(\theta, \phi)$ as already defined above. Here, $J_{0}(\vec{q})$ denotes the Fourier component of the charge density operator and $J_{ \pm 1}(\vec{q})$ the FOURIER components of the transverse current density operator. Furthermore, $\left(m_{1}, m_{2}\right)$ stands for the spin quantum numbers of the final two-nucleon state, either in the standard (coupled) representation $\left(s, m_{s}\right)$ of the total spin $s$ of the outgoing nucleons and its projection $m_{s}$ on the relative momentum, or in the helicity (uncoupled) basis $\left(\lambda_{p}, \lambda_{n}\right)$. The transformation from one representation to the other is simply given by a Clebsch-Gordan coefficient

$$
t_{\lambda_{p} \lambda_{n} \lambda \lambda_{d}}=\sum_{s m_{s}}(-)^{m_{s}} \hat{s}\left(\begin{array}{ccc}
\frac{1}{2} & \frac{1}{2} & s \\
\lambda_{p} & \lambda_{n} & -m_{s}
\end{array}\right) t_{s m_{s} \lambda \lambda_{d}}
$$

In Eq. (27a) noncovariant state normalization has been assumed and the hadronic c.m. motion has been eliminated already. Thus initial and final hadronic states refer to the relative two-body motion in the hadronic rest frame. Eq. (27b) defines the reduced $t$-matrix. If parity is conserved, it obeys the symmetry relation

$$
t_{s-m_{s}-\lambda-\lambda_{d}}=(-)^{1+s+m_{s}+\lambda+\lambda_{d}} t_{s m_{s} \lambda \lambda_{d}}
$$

for the standard representation, and

$$
t_{-\lambda_{p}-\lambda_{n}-\lambda-\lambda_{d}}=(-)^{\lambda_{p}+\lambda_{n}+\lambda+\lambda_{d}} t_{\lambda_{p} \lambda_{n} \lambda \lambda_{d}},
$$

for the helicity representation. This relation reduces the number of independent $t$-matrix elements to 18 , six for the longitudinal $(\lambda=0)$ and twelve for the transverse $(\lambda= \pm 1)$ matrix elements.

The initial state density matrix $\rho_{i}$ in (24) is a direct product of the density matrices $\rho^{\gamma}$ of the virtual photon and $\rho^{d}$ of the deuteron

$$
\rho_{i}=\rho^{\gamma} \otimes \rho^{d} .
$$

For the evaluation of $\rho^{\gamma}$ of the virtual photon, we allow the incoming electrons to be partially longitudinally polarized of degree $h$. This restriction does not mean a loss of generality because, as has been shown in [3], one obtains already for this case the maximal number of linearly independent observables. The virtual photon density matrix can be split into an unpolarized and a polarized part

$$
\rho_{\lambda \lambda^{\prime}}^{\gamma}=\rho_{\lambda \lambda^{\prime}}^{0}+h \rho_{\lambda \lambda^{\prime}}^{\prime},
$$


where $\rho^{0}$ and $\rho^{\prime}$ can be expanded in terms of independent components $\rho_{\alpha}$ and $\rho_{\alpha}^{\prime}(\alpha \in\{L, T, L T, T T\})$ according to the various combinations of longitudinal and transverse polarization

$$
\begin{aligned}
& \rho_{\lambda \lambda^{\prime}}^{0}=\sum_{\alpha=L, T, L T, T T} \delta_{\lambda \lambda^{\prime}}^{\alpha} \rho_{\alpha}, \\
& \rho_{\lambda \lambda^{\prime}}^{\prime}=\sum_{\alpha=L, T, L T, T T} \delta_{\lambda \lambda^{\prime}}^{\prime \alpha} \rho_{\alpha}^{\prime},
\end{aligned}
$$

with

$$
\begin{array}{ll}
\delta_{\lambda \lambda^{\prime}}^{L}=\delta_{\lambda \lambda^{\prime}} \delta_{\lambda 0}, & \delta_{\lambda \lambda^{\prime}}^{L T}=\lambda^{\prime} \delta_{\lambda 0}+\lambda \delta_{\lambda^{\prime} 0}, \\
\delta_{\lambda \lambda^{\prime}}^{T}=\delta_{\lambda \lambda^{\prime}}|\lambda|, & \delta_{\lambda \lambda^{\prime}}^{T T}=\delta_{\lambda,-\lambda^{\prime}}|\lambda|, \\
\delta_{\lambda \lambda^{\prime}}^{\prime L}=0, & \delta_{\lambda \lambda^{\prime}}^{L T}=\left|\lambda^{\prime}\right| \delta_{\lambda 0}+|\lambda| \delta_{\lambda^{\prime} 0}, \\
\delta_{\lambda \lambda^{\prime}}^{\prime T}=\delta_{\lambda \lambda^{\prime}} \lambda, & \delta_{\lambda \lambda^{\prime}}^{T T}=0 .
\end{array}
$$

They obey the symmetries

$$
\begin{aligned}
& \delta_{\lambda^{\prime} \lambda}^{\alpha}=\delta_{\lambda \lambda^{\prime}}^{\alpha}=(-)^{\lambda+\lambda^{\prime}} \delta_{-\lambda^{\prime}-\lambda}^{\alpha}, \\
& \delta_{\lambda^{\prime} \lambda}^{\alpha}=\delta_{\lambda \lambda^{\prime}}^{\alpha}=(-)^{1+\lambda+\lambda^{\prime}} \delta_{-\lambda^{\prime}-\lambda}^{\alpha} .
\end{aligned}
$$

The independent components $\rho_{\alpha}$ and $\rho_{\alpha}^{\prime}$ are given by the well-known expressions (note $Q^{2}=-q_{\nu}^{2}>0$ )

$$
\begin{array}{ll}
\rho_{L}=\rho_{00}^{0}=\beta^{2} Q^{2} \frac{\xi^{2}}{2 \eta}, & \rho_{T}=\rho_{11}^{0}=\frac{1}{2} Q^{2}\left(1+\frac{\xi}{2 \eta}\right), \\
\rho_{L T}=\rho_{01}^{0}=\beta Q^{2} \frac{\xi}{\eta} \sqrt{\frac{\eta+\xi}{8}}, & \rho_{T T}=\rho_{-11}^{0}=-Q^{2} \frac{\xi}{4 \eta}, \\
\rho_{L T}^{\prime}=\rho_{01}^{\prime}=\frac{1}{2} \beta \frac{Q^{2}}{\sqrt{2 \eta}} \xi, \quad \rho_{T}^{\prime}=\rho_{11}^{\prime}=\frac{1}{2} Q^{2} \sqrt{\frac{\eta+\xi}{\eta}},
\end{array}
$$

with

$$
\beta=\frac{q^{l a b}}{q^{c}}, \quad \xi=\frac{Q^{2}}{\left(q^{l a b}\right)^{2}}, \quad \eta=\tan ^{2}\left(\frac{\theta_{e}^{\text {lab }}}{2}\right),
$$

where $\beta$ expresses the boost from the lab system to the frame in which the hadronic current is evaluated and $\vec{q}^{c}$ denotes the momentum transfer in this frame. If, as is the case here, one calculates the observables in the final $n p$-c.m. system, one has $\vec{q}^{c}=\vec{q}^{c . m}$. We further note the simple relation to the often used parametrization of the virtual photon density matrix in terms of the quantities $v_{\alpha^{(\prime)}}$ of Ref. [14] (for $\beta=1$ )

$$
\rho_{\alpha}^{(\prime)}=\frac{Q^{2}}{2 \eta} v_{\alpha^{(\prime)}}
$$

where $\alpha \in\{L, T, L T, T T\}$.

Furthermore, the deuteron density matrix $\rho^{d}$ can be expressed in terms of irreducible spin operators $\tau^{[I]}$ with respect to the deuteron spin space

$$
\rho_{\lambda_{d} \lambda_{d}}^{d}=\frac{1}{3} \sum_{I M}(-)^{M} \hat{I}\left\langle 1 \lambda_{d}\left|\tau_{M}^{[I]}\right| 1 \lambda_{d}^{\prime}\right\rangle P_{I-M}^{d},
$$

where $P_{00}^{d}=1$, and $P_{1-M}^{d}$ and $P_{2-M}^{d}$ describe vector and tensor polarization components of the deuteron, respectively. We use throughout the notation $\hat{I}=\sqrt{2 I+1}$. The spin operators are defined by their reduced matrix elements

$$
\left\langle 1|| \tau^{[I]} \| 1\right\rangle=\sqrt{3} \hat{I} \quad \text { for } \quad I=0,1,2 .
$$

From now on we will assume that the deuteron density matrix is diagonal with respect to an orientation axis $\vec{d}$ having spherical angles $\left(\theta_{d}, \phi_{d}\right)$ with respect to the coordinate system associated with the scattering plane in the lab frame (see Fig. 1). Then one has with respect to $\vec{d}$ as quantization axis

$$
\rho_{m m^{\prime}}^{d}=p_{m} \delta_{m m^{\prime}}
$$


where $p_{m}$ denotes the probability for finding a deuteron spin projection $m$ on the orientation axis. With respect to this axis one has $P_{I M}^{d}(\vec{d})=P_{I}^{d} \delta_{M, 0}$, where the orientation parameters $P_{I}^{d}$ are related to the $p_{m}$ by

$$
\begin{aligned}
P_{I}^{d} & =\sqrt{3} \hat{I} \sum_{m}(-)^{1-m}\left(\begin{array}{ccc}
1 & 1 & I \\
m & -m & 0
\end{array}\right) p_{m} \\
& =\delta_{I, 0}+\sqrt{\frac{3}{2}}\left(p_{1}-p_{-1}\right) \delta_{I, 1}+\frac{1}{\sqrt{2}}\left(1-3 p_{0}\right) \delta_{I, 2} .
\end{aligned}
$$

The polarization components in the chosen lab frame are obtained from the $P_{I}^{d}$ by a rotation

$$
P_{I M}^{d}(\vec{z})=P_{I}^{d} e^{i M \phi_{d}} d_{M 0}^{I}\left(\theta_{d}\right),
$$

where $d_{m m^{\prime}}^{j}$ denotes a small rotation matrix [15]. Thus the deuteron density matrix becomes finally

$$
\rho_{\lambda_{d} \lambda_{d}}^{d}=\frac{1}{\sqrt{3}}(-)^{1-\lambda_{d}} \sum_{I M} \hat{I}\left(\begin{array}{ccc}
1 & 1 & I \\
\lambda_{d}^{\prime} & -\lambda_{d} & M
\end{array}\right) P_{I}^{d} e^{-i M \phi_{d}} d_{M 0}^{I}\left(\theta_{d}\right)
$$

This means, the deuteron target is characterized by four parameters, namely the vector and tensor polarizations $P_{1}^{d}$ and $P_{2}^{d}$, respectively, and by the orientation angles $\theta_{d}$ and $\phi_{d}$. Note that the deuteron density matrix undergoes no change in the transformation from the lab to the c.m. system, since the boost to the c.m. system is collinear with the deuteron quantization axis [16].

Now we turn to the definition of the operator $\Omega_{X}$ characterizing the various observables. One has 16 independent observables according to all combinations of the four operators $\left(\mathbb{1}_{2}, \vec{\sigma}\right)$ in the spin space of each of the two nucleons. In detail, if no polarization analysis of the outgoing nucleons is performed, one has

$$
\Omega_{1}=\Omega_{00}=\sigma_{0}(p) \otimes \sigma_{0}(n),
$$

where we have defined $\sigma_{0}=\mathbb{1}_{2}$. If the polarization component $x_{i}$ of the outgoing proton or neutron, respectively, is measured, the corresponding operator is

$$
\Omega_{i 0}=\sigma_{i}(p) \otimes \sigma_{0}(n) \quad \text { or } \quad \Omega_{0 i}=\sigma_{0}(p) \otimes \sigma_{i}(n) .
$$

Finally, the combined measurement of the polarization components $x_{i}(p)$ and $x_{j}(n)$ of both final particles is represented by

$$
\Omega_{i j}=\sigma_{i}(p) \otimes \sigma_{j}(n) .
$$

Thus each observable $X$ is represented by a pair $X=\left(\alpha^{\prime} \alpha\right)$ with $\alpha^{\prime}, \alpha=0, \ldots, 3$ and related to the operator $\Omega_{\alpha^{\prime} \alpha}=\sigma_{\alpha^{\prime}}(p) \otimes \sigma_{\alpha}(n)$. Since the $T$-matrix is calculated in the $n p$-c.m. system, the spin operators of both particles refer to the same reference frame with $z$-axis parallel to $\vec{p}_{n p}$ and $y$-axis along $\vec{q} \times \vec{p}_{n p}$, i.e., perpendicular to the reaction plane. Thus the polarization components of the proton are chosen according to the Madison convention while for the neutron the $y$ - and $z$-components of $\vec{P}$ have to be reversed in order to comply with this convention. The resulting observables are listed in Table I and are divided into two sets, called $A$ and $B$, according to their behaviour under a parity transformation $[8]$.

TABLE I: Notation for the cartesian components of the spin observables and their division into sets $A$ and $B$.

\begin{tabular}{cccccccccc}
\hline \hline observable & 1 & $x_{p}$ & $y_{p}$ & $z_{p}$ & $x_{n}$ & $y_{n}$ & $z_{n}$ & \\
set & $A$ & $B$ & $A$ & $B$ & $B$ & $A$ & $B$ & $z_{p}$ \\
\hline observable & $x_{p} x_{n}$ & $x_{p} y_{n}$ & $x_{p} z_{n}$ & $y_{p} x_{n}$ & $y_{p} y_{n}$ & $y_{p} z_{n}$ & $z_{p} x_{n}$ & $z_{p} z_{n}$ \\
set & $A$ & $B$ & $A$ & $B$ & $A$ & $B$ & $A$ & $B$ \\
\hline \hline
\end{tabular}

For real photons, the photon density matrix contains transverse components only, and thus, in order to obtain the corresponding observables in photodisintegration, one has to make in (24) the replacements

$$
\begin{array}{ccc}
\rho_{L} \rightarrow 0, & \rho_{L T} \rightarrow 0, & \rho_{L T}^{\prime} \rightarrow 0, \\
c\left(k_{1}^{\text {lab }}, k_{2}^{\text {lab }}\right) \rho_{T} \rightarrow 1 / 6, & h c\left(k_{1}^{\text {lab }}, k_{2}^{\text {lab }}\right) \rho_{T}^{\prime} \rightarrow-P_{c}^{\gamma} / 6, & c\left(k_{1}^{\text {lab }}, k_{2}^{\text {lab }}\right) \rho_{T T} \rightarrow P_{l}^{\gamma} / 6,
\end{array}
$$

where $P_{l}^{\gamma}$ and $P_{c}^{\gamma}$ denote the degree of linear and circular photon polarization, respectively, and $P_{l}^{\gamma}<0$ means linear polarization along the $x$-axis while along the $y$-axis for $P_{l}^{\gamma}>0$. Furthermore, $P_{c}^{\gamma}>0$ or $P_{c}^{\gamma}<0$ describe right or left handed circular polarization, respectively. 


\section{Structure functions}

For each observable $X$ a set of structure functions is defined as quadratic hermitean forms of the $t$-matrix elements by

$$
\begin{aligned}
f_{L}^{I M}(X) & =\frac{2}{1+\delta_{M 0}} \Re e\left(i^{\bar{\delta}_{I}^{X}} \mathcal{U}_{X}^{00 I M}\right) \\
f_{T}^{I M}(X) & =\frac{4}{1+\delta_{M 0}} \Re e\left(i^{\delta_{I}^{X}} \mathcal{U}_{X}^{11 I M}\right) \\
f_{L T}^{I M \pm}(X) & =\frac{4}{1+\delta_{M 0}} \Re e\left[i^{\delta_{I}^{X}}\left(\mathcal{U}_{X}^{01 I M} \pm(-)^{I+M+\delta_{X}, B} \mathcal{U}_{X}^{01 I-M}\right)\right] \\
f_{T T}^{I M \pm}(X) & =\frac{2}{1+\delta_{M 0}} \Re e\left[i^{\delta_{I}^{X}}\left(\mathcal{U}_{X}^{-11 I M} \pm(-)^{I+M+\delta_{X}, B} \mathcal{U}_{X}^{-11 I-M}\right)\right] \\
f_{T}^{\prime I M}(X) & =\frac{4}{1+\delta_{M 0}} \Re e\left(i^{1+\bar{\delta}_{I}^{X}} \mathcal{U}_{X}^{11 I M}\right) \\
f_{L T}^{\prime I M \pm}(X) & =\frac{4}{1+\delta_{M 0}} \Re e\left[i^{1+\bar{\delta}_{I}^{X}}\left(\mathcal{U}_{X}^{01 I M} \pm(-)^{I+M+\delta_{X}, B} \mathcal{U}_{X}^{01 I-M}\right)\right]
\end{aligned}
$$

Here $\bar{\delta}_{I}^{X}$ is defined by

$$
\bar{\delta}_{I}^{X}=\left(\delta_{X, B}-\delta_{I 1}\right)^{2}, \text { with } \delta_{X, B}:=\left\{\begin{array}{ll}
0 & \text { for } X \in A \\
1 & \text { for } X \in B
\end{array}\right\},
$$

distinguishing the two sets of observables $A$ and $B$. In the foregoing expressions, the $\mathcal{U}$ 's are given as bilinear hermitean forms in the reduced $t$-matrix elements, i.e., for $X=\left(\alpha^{\prime} \alpha\right)$

$$
\mathcal{U}_{\alpha^{\prime} \alpha}^{\lambda^{\prime} \lambda I M}=\sum_{m_{1}^{\prime} m_{2}^{\prime} \lambda_{d}^{\prime} m_{1} m_{2} \lambda_{d}} t_{m_{1}^{\prime} m_{2}^{\prime} \lambda^{\prime} \lambda_{d}^{\prime}}^{*}\left\langle m_{1}^{\prime} m_{2}^{\prime}\left|\sigma_{\alpha^{\prime}}(p) \sigma_{\alpha}(n)\right| m_{1} m_{2}\right\rangle t_{m_{1} m_{2} \lambda \lambda_{d}}\left\langle\lambda_{d}\left|\tau_{M}^{[I]}\right| \lambda_{d}^{\prime}\right\rangle
$$

Although the $\mathcal{U}$ 's are independent of the chosen representation for the matrix elements, their explicit form in terms of the $t$-matrix elements depends certainly on the representation for the initial and final spin states. We have already mentioned that two conventions are in common use, the helicity representation with spin quantum numbers $\left(\lambda_{p}, \lambda_{n}\right)$ and the standard one with $\left(s, m_{s}\right)$. A third representation called hybrid basis, where the quantization axis is chosen perpendicular to the reaction plane, was introduced in [9]. Explicit expressions are listed in the Appendix A for the $\left(s, m_{s}\right)$-representation. More general representations, which are obtained by arbitrary rotations of the quantization axes of initial and final spin states, are considered in [6]. However one should keep in mind that the observables and thus the structure functions are independent of the representation because they are defined as traces over the spin degrees of freedom (see (24)).

Note that $f_{\alpha}^{00-}(X), f_{\alpha}^{20-}(X)$ and $f_{\alpha}^{10+}(X)$ vanish identically for $X \in A$ and correspondingly $f_{\alpha}^{00+}(X), f_{\alpha}^{20+}(X)$ and $f_{\alpha}^{10-}(X)$ for $X \in B$. For this reason we often use the notation $f_{\alpha}(X), f_{\alpha}^{10}(X)$ and $f_{\alpha}^{20}(X)$ instead of $f_{\alpha}^{00 \pm}(X)$, $f_{\alpha}^{10 \mp}(X)$ and $f_{\alpha}^{20 \pm}(X)$, respectively.

The structure functions $f_{\alpha}^{(\prime) I M( \pm)}(X)(\alpha \in\{L, T, L T, T T\})$ (primed and unprimed structure functions $f_{\alpha}^{\prime I M( \pm)}(X)$ and $f_{\alpha}^{I M( \pm)}(X)$ are here referred to collectively as $\left.f_{\alpha}^{(\prime) I M( \pm)}(X)\right)$ contain the complete information on the dynamical properties of the $n p$-system available in deuteron electrodisintegration. They are functions of the $n p$-angle $\theta$, the relative $n p$-energy $E_{n p}$, and the three-momentum transfer squared $\left(q^{\text {c.m. }}\right)^{2}$, all in the c.m. system.

In terms of these structure functions a general observable in $d\left(e, e^{\prime} N\right) N$ and $d\left(e, e^{\prime} n p\right)$ is given by

$$
\begin{aligned}
\mathcal{O}\left(\Omega_{X}\right)=c\left(k_{1}^{\mathrm{lab}}, k_{2}^{\mathrm{lab}}\right) \sum_{I=0}^{2} P_{I}^{d} \sum_{M=0}^{I}\{ & \left(\rho_{L} f_{L}^{I M}(X)+\rho_{T} f_{T}^{I M}(X)+\rho_{L T} f_{L T}^{I M+}(X) \cos \phi\right. \\
& \left.+\rho_{T T} f_{T T}^{I M+}(X) \cos 2 \phi\right) \cos \left(M \tilde{\phi}-\bar{\delta}_{I}^{X} \frac{\pi}{2}\right) \\
& -\left(\rho_{L T} f_{L T}^{I M-}(X) \sin \phi+\rho_{T T} f_{T T}^{I M-}(X) \sin 2 \phi\right) \sin \left(M \tilde{\phi}-\bar{\delta}_{I}^{X} \frac{\pi}{2}\right) \\
& +h\left[\left(\rho_{T}^{\prime} f_{T}^{\prime I M}(X)+\rho_{L T}^{\prime} f_{L T}^{\prime I M-}(X) \cos \phi\right) \sin \left(M \tilde{\phi}-\bar{\delta}_{I}^{X} \frac{\pi}{2}\right)\right. \\
& \left.\left.+\rho_{L T}^{\prime} f_{L T}^{I I M+}(X) \sin \phi \cos \left(M \tilde{\phi}-\bar{\delta}_{I}^{X} \frac{\pi}{2}\right)\right]\right\} d_{M 0}^{I}\left(\theta_{d}\right),
\end{aligned}
$$


where we have introduced $\tilde{\phi}=\phi-\phi_{d}$. In particular, one obtains for $X=1$ and $P_{I}^{d}=\delta_{I, 0}$ the unpolarized cross section as

$$
S_{0}=c\left(k_{1}^{\mathrm{lab}}, k_{2}^{\mathrm{lab}}\right)\left(\rho_{L} f_{L}+\rho_{T} f_{T}+\rho_{L T} f_{L T} \cos \phi+\rho_{T T} f_{T T} \cos 2 \phi\right),
$$

using as a shorthand $f_{\alpha}=f_{\alpha}^{00+}(1)$. One should remember that the nucleon angles and polarization components refer to the c.m. frame. The transformation to the lab frame is described in the previous subsection.

TABLE II: Listing of $(I M)$ - and $(I M \pm)$-values of nonvanishing structure functions $f_{\alpha}^{(\prime) I M}(X)$ and $f_{\alpha}^{(\prime) I M \pm}(X)$, respectively.

\begin{tabular}{cccccccc}
\hline \hline \multicolumn{2}{c}{$f_{L}^{I M}(X), f_{T}^{I M}(X)$} & \multicolumn{2}{c}{$f_{T}^{\prime I M}(X)$} & \multicolumn{3}{c}{$f_{L T}^{I M \pm}(X), f_{T T}^{I M \pm}(X), f_{L T}^{\prime I M \pm}(X)$} \\
\hline$X \in A$ & $X \in B$ & $X \in A$ & $X \in B$ & \multicolumn{2}{c}{$X \in A$} & $X \in B$ \\
\hline 00 & 10 & 10 & 00 & $00+$ & $10-$ & $10+$ & $00-$ \\
11 & 11 & 11 & 11 & $11+$ & $11-$ & $11+$ & $11-$ \\
20 & 21 & 21 & 20 & $20+$ & $21-$ & $21+$ & $20-$ \\
21 & 22 & 21 & $21+$ & $22-$ & $22+$ & $21-$ \\
22 & 22 & & 22 & $22+$ & & & $22-$ \\
\hline \hline
\end{tabular}

TABLE III: Number of nonvanishing structure functions $f_{\alpha}^{(\prime) I M}(X)$ and $f_{\alpha}^{(\prime) I M \pm}(X)$ for an observable $X \in A$ or $X \in B$.

\begin{tabular}{cccccccc}
\hline \hline set & $L$ & $T$ & $T^{\prime}$ & $L T$ & $L T^{\prime}$ & $T T$ & total \\
\hline$A$ & 5 & 5 & 4 & 9 & 9 & 9 & 41 \\
$B$ & 4 & 4 & 5 & 9 & 9 & 9 & 40 \\
\hline \hline
\end{tabular}

The possible $(I, M)$-values are listed in Table II and the total number of structure functions for each observable $X$ and each $\alpha$ are listed in Table III. As mentioned in the introduction, one finds altogether a total number of 648 observables, each of which is a hermitean form of the $t$-matrix elements. However, since the $t$-matrix has only $n=18$ independent complex amplitudes, only $n^{2}=324$ linearly independent hermitean forms can exist. Indeed, one finds $n^{2}$ linear relations between the observables which are presented in the next section. The remaining structure functions are linearly independent so that indeed the maximal information can be obtained by using longitudinally polarized electrons alone. Transverse polarization is not necessary.

On the other hand, since each reaction matrix element is in general a complex number, but one overall phase is undetermined, a set of $2 n-1$ properly chosen observables should suffice to determine completely all matrix elements. This seeming contradiction is resolved by the observation, that the linearly independent observables are not completely independent of each other in a more general sense. In fact, any bilinear form $t_{j}^{*}, t_{j}$ can be expressed as a linear form in the observables (see [5] and also Sec. IID), and for these bilinear forms one can find exactly $(n-1)^{2}$ quadratic relations (see Appendix B), thus reducing the total number of independent observables just to the required number $2 n-1$. Consequently, one can determine all matrix elements from $2 n-1$ properly chosen observables. However, one should keep in mind that the solution is in general not unique but contains discrete ambiguities. This is discussed in Sect. III.

To close this section, we will give for the transverse structure functions $\left(\alpha \in\left\{T, T^{\prime}, T T\right\}\right)$ the correspondence to the observables in photodisintegration derived in [8]. The formal definition of observables is completely analogous except for the fact that in photodisintegration only transverse current components contribute. Taking into account the slightly different definition of the $T$-matrix (compare $T$ of $(27 \mathrm{a})$ with the definition of $T^{\gamma}$ in $[17,18]$ ), i.e.

$$
T_{m_{1} m_{2} \lambda \lambda_{d}}(\theta, \phi)=\sqrt{\frac{W_{n p} q^{c . m .}}{M_{d}}} T_{m_{1} m_{2} \lambda \lambda_{d}}^{\gamma}(\theta, \phi)
$$

one has the following relations at the photon point with respect to the general form of an observable in photodisint- 
TABLE IV: Definition of $X^{\prime}(X)$ and $p(X)$.

\begin{tabular}{cccccccc}
\hline \hline$X$ & 1 & $x_{p} x_{n}$ & $x_{p} z_{n}$ & $y_{p}$ & $x_{p}$ & $x_{n}$ & $z_{p}$ \\
\hline$X^{\prime}(X)$ & $y_{p} y_{n}$ & $z_{p} z_{n}$ & $z_{p} x_{n}$ & $y_{n}$ & $z_{p} y_{n}$ & $y_{p} z_{n}$ & $x_{p} y_{n}$ \\
$p(X)$ & 1 & -1 & 1 & 1 & -1 & -1 & 1 \\
\hline set & $A$ & $A$ & $A$ & $A$ & $B$ & $B$ & $B$ \\
\hline \hline
\end{tabular}

gration as given in (14) and (15) of [8]

$$
\begin{aligned}
f_{T}^{I M}(X) & =(-)^{\bar{\delta}_{I}^{X}} \frac{6 W_{n p} q^{c . m .}}{M_{d}} P_{X}^{0, I M} \frac{d \sigma}{d \Omega_{n p}}, \\
f_{T}^{\prime I M}(X) & =-\frac{6 W_{n p} q^{c . m .}}{M_{d}} P_{X}^{c, I M} \frac{d \sigma}{d \Omega_{n p}}, \\
f_{T T}^{I M \pm}(X) & =\mp(-)^{\bar{\delta}_{I}^{X}} \frac{W_{n p} q^{c . m} .}{M_{d}} \frac{6}{1+\delta_{M 0}}\left(P_{X}^{l, I M} \pm(-)^{I+M+\delta_{X, B}} P_{X}^{l, I-M}\right) \frac{d \sigma}{d \Omega_{n p}} .
\end{aligned}
$$

\section{Linear relations between structure functions}

As is shown in detail in [3], the derivation of linear relations among observables is based on the inversion of (51) expressing any bilinear form $t_{s^{\prime} m_{s}^{\prime} \lambda^{\prime} m^{\prime}}^{*} t_{s m_{s} \lambda m}$ as a linear superposition of observables. This inversion can be done analytically (see [3]). In general one obtains two types of relations among the structure functions of an observable $X$ and those of another observable $X^{\prime}(X)$, uniquely related to $X$. Explicitly, one finds as the first type of equations

$$
\begin{aligned}
g^{00}(X) & =\frac{1}{3} p(X)\left(\bar{g}^{00}\left(X^{\prime}\right)-\sqrt{2} \bar{g}^{20}\left(X^{\prime}\right)-\sqrt{3} \bar{g}^{22}\left(X^{\prime}\right)\right) \\
g^{11}(X) & =p(X) \bar{g}^{11}\left(X^{\prime}\right) \\
g^{20}(X) & =\frac{1}{3} p(X)\left(-\sqrt{2} \bar{g}^{00}\left(X^{\prime}\right)+2 \bar{g}^{20}\left(X^{\prime}\right)-\sqrt{\frac{3}{2}} \bar{g}^{22}\left(X^{\prime}\right)\right) \\
g^{21}(X) & =p(X) \bar{g}^{21}\left(X^{\prime}\right) \\
g^{22}(X) & =-\frac{2}{\sqrt{3}} p(X)\left(\bar{g}^{00}\left(X^{\prime}\right)+\frac{1}{\sqrt{2}} \bar{g}^{20}\left(X^{\prime}\right)\right) .
\end{aligned}
$$

The second type of equations reads

$$
\begin{aligned}
& h^{10}(X)=-\frac{1}{\sqrt{2}} p(X) \bar{h}^{22}\left(X^{\prime}\right), \\
& h^{11}(X)=p(X) \bar{h}^{21}\left(X^{\prime}\right) \\
& h^{21}(X)=-p(X) \bar{h}^{11}\left(X^{\prime}\right) \\
& \left.h^{22}(X)=\sqrt{2} p(X) \bar{h}^{10}\left(X^{\prime}\right)\right)
\end{aligned}
$$

where $X^{\prime}(X)$ and $p(X)$ are listed in Table IV for 8 observables. For the remaining other 8 observables, not listed in Table IV, one obtains $X^{\prime}(X)$ from Table IV with the help of the relation

$$
X^{\prime}\left(X^{\prime}(X)\right)=X,
$$

and $p(X)$ from the relation

$$
p\left(X^{\prime}(X)\right)=(-)^{\delta_{X, B}} p(X) .
$$

Table $\mathrm{V}$ shows which of these two types of relations holds for a specific structure function, depending on whether $X$ belongs to an observable of set $A$ or $B$. Which structure functions are related to each other in these relations, i.e., $g^{I M}(X)$ to $\bar{g}^{I^{\prime} M^{\prime}}\left(X^{\prime}\right)$ and $h^{I M}(X)$ to $\bar{h}^{I^{\prime} M^{\prime}}\left(X^{\prime}\right)$, is also listed in Table V.

At the end of this section, we will give two examples of how to find the proper relation for a given structure function of an observable $X$. As first example we choose the $y$-component of the neutron polarization, i.e. $X=y_{n}$ belonging 
TABLE V: Listing of structure functions $g^{I M}(X)$ and $\bar{g}^{I^{\prime} M^{\prime}}\left(X^{\prime}\right)$ for observables $X, X^{\prime}$ which fulfill the relations (56), and of structure functions $h^{I M}(X)$ and $\bar{h}^{I^{\prime} M^{\prime}}\left(X^{\prime}\right)$ for observables which fulfill the relations (57). The associated observable $X^{\prime}(X)$ is either listed in Table IV or can be obtained using (58).

\begin{tabular}{cccccc}
\hline \hline & \multicolumn{5}{c}{$X \in A[B]$} \\
\hline$g[\bar{h}]^{I M}(X)$ & $f_{L}^{I M}$ & $f_{L T}^{I M+}$ & $f_{L T}^{\prime M+}$ & $f_{T}^{I M}$ & $f_{T T}^{I M+}$ \\
$\bar{g}[\bar{h}]^{I^{\prime} M^{\prime}}\left(X^{\prime}\right)$ & $f_{L}^{I^{\prime} M^{\prime}}$ & $f_{L T}^{I^{\prime} M^{\prime}+}$ & $f_{L T}^{\prime I^{\prime} M^{\prime}+}$ & $-f_{T T}^{I^{\prime} M^{\prime}+}$ & $-f_{T}^{I^{\prime} M^{\prime}}$ \\
\hline & & $X \in B[A]$ & \\
\hline$g[h]^{I M}(X)$ & $f_{L T}^{I M-}$ & $f_{L T}^{\prime I M-}$ & $f_{T}^{\prime I M}$ & $f_{T T}^{I M-}$ \\
$\bar{g}[\bar{h}]^{I^{\prime} M^{\prime}}\left(X^{\prime}\right)$ & $f_{L T}^{I^{\prime} M^{\prime}-}$ & $-f_{L T}^{I^{\prime} M^{\prime}-}$ & $f_{T T}^{I^{\prime} M^{\prime}-}$ & $-f_{T}^{\prime I^{\prime} M^{\prime}}$ \\
\hline \hline
\end{tabular}

TABLE VI: Listing of the matrix $U_{l s \mu}^{j}$.

\begin{tabular}{ccccc}
\hline \hline$l$ & $s$ & $\mu=1$ & 2 & 3 \\
\hline$j-1$ & 1 & $\cos \epsilon_{j}$ & 0 & $-\sin \epsilon_{j}$ \\
$j$ & 0 & 0 & 1 & 0 \\
$j+1$ & 1 & $\sin \epsilon_{j}$ & 0 & 0 \\
$j$ & 1 & 0 & 0 & 0 \\
\hline \hline
\end{tabular}

to set $A$. According to Table IV its structure functions are related to the ones of the $y$-component of the proton polarization. With the help of (58) and (59) one finds $X^{\prime}\left(y_{n}\right)=y_{p}$ and $p\left(y_{n}\right)=1$ and in view of Table $\mathrm{V}$ the relations (56) apply, e.g.

$$
\begin{aligned}
& f_{L}^{00}\left(y_{n}\right)=\frac{1}{3}\left(f_{L}^{00}\left(y_{p}\right)-\sqrt{2} f_{L}^{20}\left(y_{p}\right)-\sqrt{3} f_{L}^{22}\left(y_{p}\right)\right), \\
& f_{T}^{00}\left(y_{n}\right)=-\frac{1}{3}\left(f_{T T}^{00}\left(y_{p}\right)-\sqrt{2} f_{T T}^{20}\left(y_{p}\right)-\sqrt{3} f_{T T}^{22}\left(y_{p}\right)\right) .
\end{aligned}
$$

For the second example we choose $X=z_{p} y_{n}$, belonging to set $B$, and the structure function $f_{L}^{10}\left(z_{p} y_{n}\right)$. From Table IV with the help of (58) and (59) one finds $X^{\prime}\left(z_{p} y_{n}\right)=x_{p}$ and $p\left(z_{p} y_{n}\right)=1$. Furthermore, according to Table V the relation (57) applies, resulting, for example, in

$$
f_{L}^{10}\left(z_{p} y_{n}\right)=-\frac{1}{\sqrt{2}} f_{L}^{22}\left(x_{p}\right)
$$

\section{E. Multipole decomposition}

A convenient parametrization of the angular dependence of observables and structure functions is provided by an expansion in terms of the small rotation matrices $d_{m^{\prime} m}^{j}[7,19-21]$. Explicit expressions for deuteron electrodisintegration have been derived recently in [10]. They facilitate the analysis of the contributions of the various charge, electric, and magnetic transition multipole moments to the different structure functions. This expansion is based on the multipole expansion of the $t$-matrix. We take the outgoing $n p$-state in the form of the Blatt-Biedenharn convention $[22]$

$$
\left|\vec{p} s m_{s}\right\rangle^{(-)}=\sum_{\mu j m_{j} l} \hat{l}\left(l 0 s m_{s} \mid j m_{s}\right) e^{-i \delta_{\mu}^{j}} U_{l s \mu}^{j} D_{m_{j} m_{s}}^{j}(R)\left|\mu j m_{j}\right\rangle,
$$

where $D_{m_{j} m_{s}}^{j}(R)$ denote the rotation matrices in the convention of Rose [15] and $\mu=1, \ldots, 4$ numbers the four possible partial waves for a given total angular momentum $j>0$. For $j=0$ one has only two partial waves. The phase shifts are denoted by $\delta_{\mu}^{j}$, and the matrix $U_{l s \mu}^{j}$ is determined by the mixing parameters $\epsilon_{j}$ as listed in Table VI.

Furthermore, $R=(0,-\theta,-\phi)$ rotates the chosen quantization axis into the direction of the relative $n p$-momentum $\vec{p}$. The partial waves

$$
\left|\mu j m_{j}\right\rangle=\sum_{l^{\prime} s^{\prime}} U_{l^{\prime} s^{\prime} \mu}^{j}\left|\mu\left(l^{\prime} s^{\prime}\right) j m_{j}\right\rangle
$$


TABLE VII: Listing of the sets $\kappa_{X}$ determining the summation values $\kappa$ in the multipole decomposition (73) of a structure function for an observable $X=\left(\alpha^{\prime} \alpha\right)$.

\begin{tabular}{|c|c|c|c|c|c|c|c|c|c|c|c|c|c|c|c|c|}
\hline$\alpha^{\prime}$ & 0 & 3 & 0 & 3 & 1 & 0 & 2 & 0 & 3 & 1 & 3 & 2 & 1 & 2 & 1 & 2 \\
\hline$\alpha$ & 0 & 0 & 3 & 3 & 0 & 1 & 0 & 2 & 1 & 3 & 2 & 3 & 1 & 1 & 2 & 2 \\
\hline$\kappa_{X}$ & \multicolumn{4}{|c|}{0} & \multicolumn{8}{|c|}{$-1,1$} & \multicolumn{4}{|c|}{$-2,0,2$} \\
\hline
\end{tabular}

are solutions of a system of coupled equations of $N N$-scattering. In this convention, the $t$-matrix reads

$$
t_{s m_{s} \lambda \lambda_{d}}(\theta)=(-)^{\lambda} \sqrt{1+\delta_{\lambda 0}} \sum_{L l j m_{j} \mu} \frac{\hat{\jmath}}{\hat{\jmath}}\left(1 \lambda_{d} L \lambda \mid j m_{j}\right)\left(l 0 s m_{s} \mid j m_{s}\right) \mathcal{O}^{L \lambda}(\mu j l s) d_{m_{j} m_{s}}^{j}(\theta),
$$

with

$$
\mathcal{O}^{L \lambda}(\mu j l s)=\sqrt{4 \pi} e^{i \delta_{\mu}^{j}} U_{l s, \mu}^{j} N_{\lambda}^{L}(\mu j)
$$

and

$$
N_{\lambda}^{L}(\mu j)=\delta_{|\lambda| 1}\left(E^{L}(\mu j)+\lambda M^{L}(\mu j)\right)+\delta_{\lambda 0} C^{L}(\mu j),
$$

where $E^{L}(\mu j), M^{L}(\mu j)$ and $C^{L}(\mu j)$ denote the reduced electric, magnetic and charge multipole matrix elements, respectively, between the deuteron state and a final state partial wave $|\mu j\rangle$ in the Blatt-Biedenharn parametrization. Parity conservation implies the selection rules

$$
\begin{aligned}
(C / E)^{L}(\mu j) & =0 \text { for } \quad(-)^{L+j+\mu}=-1 \\
M^{L}(\mu j) & =0 \text { for } \quad(-)^{L+j+\mu}=1
\end{aligned}
$$

which leads to the relation

$$
\mathcal{O}^{L-\lambda}(\mu j l s)=(-)^{L+l} \mathcal{O}^{L \lambda}(\mu j l s) .
$$

As is shown in detail in [10], one obtains for a structure function the general multipole expansion according to the expressions in (49) from the one of $\mathcal{U}_{\alpha^{\prime} \alpha}^{\lambda^{\prime} \lambda I M}$ in (51) which reads

$$
\mathcal{U}_{\alpha^{\prime} \alpha}^{\lambda^{\prime} \lambda I M}=\sum_{K, \kappa \in \kappa X} \mathcal{U}_{\alpha^{\prime} \alpha}^{\lambda^{\prime} \lambda I M, K \kappa} d_{\lambda^{\prime}-\lambda-M, \kappa}^{K}(\theta)
$$

where $d_{m^{\prime} m}^{j}(\theta)$ denotes the small $d$-function of the rotation matrices.

The sets $\kappa_{X}$ of the possible $\kappa$-values are listed in Table VII and the coefficients are given by

$$
\mathcal{U}_{\alpha^{\prime} \alpha}^{\lambda^{\prime} \lambda I M, K \kappa}=4 \pi i^{\delta_{\left(\alpha^{\prime}, \alpha\right)}^{(2)}} \sum_{L^{\prime} L \mu^{\prime} j^{\prime} \mu j} \mathcal{C}^{\lambda^{\prime} \lambda I M K}\left(L^{\prime} j^{\prime} L j\right) \widetilde{\mathcal{D}}_{\alpha^{\prime} \alpha}^{K \kappa}\left(\mu^{\prime} j^{\prime} \mu j\right) \widetilde{N}_{\lambda^{\prime}}^{L^{\prime} *}\left(\mu^{\prime} j^{\prime}\right) \widetilde{N}_{\lambda}^{L}(\mu j),
$$

where we have defined

$$
\delta_{\left(\alpha^{\prime}, \alpha\right)}^{(k)}=\delta_{\alpha^{\prime}, k}+\delta_{\alpha, k}
$$

and

$$
\begin{aligned}
\mathcal{C}^{\lambda^{\prime} \lambda I M, K}\left(L^{\prime} j^{\prime} L j\right)= & (-)^{\lambda^{\prime}+L} 2 \sqrt{3\left(1+\delta_{\lambda^{\prime} 0}\right)\left(1+\delta_{\lambda 0}\right)} \hat{\jmath}^{\prime} \hat{\jmath} \hat{I} \hat{K}^{2} \\
& \sum_{J} \hat{J}^{2}\left(\begin{array}{ccc}
J & I & K \\
\lambda-\lambda^{\prime} & M & \lambda^{\prime}-\lambda-M
\end{array}\right)\left(\begin{array}{ccc}
L^{\prime} & L & J \\
\lambda^{\prime} & -\lambda & \lambda-\lambda^{\prime}
\end{array}\right)\left\{\begin{array}{ccc}
j^{\prime} & j & K \\
L^{\prime} & L & J \\
1 & 1 & I
\end{array}\right\}, \\
\widetilde{\mathcal{D}}_{\alpha^{\prime} \alpha}^{K \kappa}\left(\mu^{\prime} j^{\prime} \mu j\right)= & (-i)^{\delta_{\left(\alpha^{\prime}, \alpha\right)}^{(2)}} \sum_{l^{\prime} s^{\prime} l s} \mathcal{D}_{\alpha^{\prime} \alpha}^{K \kappa}\left(j^{\prime} l^{\prime} s^{\prime} j l s\right) U_{l^{\prime} s^{\prime}, \mu^{\prime}}^{j^{\prime}} U_{l s, \mu}^{j} .
\end{aligned}
$$


TABLE VIII: Listing of the values of $\beta(\alpha)$ in the multipole decomposition (73).

\begin{tabular}{cccc}
\hline \hline$\alpha$ & $L / T$ & $L T$ & $T T$ \\
\hline$\beta(\alpha)$ & 0 & 1 & 2 \\
\hline \hline
\end{tabular}

with

$$
\begin{aligned}
\mathcal{D}_{\alpha^{\prime} \alpha}^{K \kappa}\left(j^{\prime} l^{\prime} s^{\prime} j l s\right)= & (-)^{l+s^{\prime}+s} \hat{l}^{\prime} \hat{l} \hat{s}^{\prime} \hat{s} \sum_{\tau^{\prime} \nu^{\prime} \tau \nu}(-)^{\tau^{\prime}+\tau} \hat{\tau}^{\prime} \hat{\tau} s_{\alpha^{\prime} \tau^{\prime} \nu^{\prime}} s_{\alpha}^{\tau \nu}\left[\sum_{S} \hat{S}^{2}\left(\begin{array}{ccc}
\tau^{\prime} & \tau & S \\
\nu^{\prime} & \nu & -\kappa
\end{array}\right)\left(\begin{array}{ccc}
\frac{1}{2} & \frac{1}{2} & \tau^{\prime} \\
\frac{1}{2} & \frac{1}{2} & \tau \\
s^{\prime} & s & S
\end{array}\right\}\right. \\
& {\left.\left[\sum_{K^{\prime}} \hat{K}^{\prime 2}\left(\begin{array}{ccc}
S & K & K^{\prime} \\
\kappa & -\kappa & 0
\end{array}\right)\left(\begin{array}{ccc}
K^{\prime} & l & l^{\prime} \\
0 & 0 & 0
\end{array}\right)\left\{\begin{array}{ccc}
S & K & K^{\prime} \\
s & j & l \\
s^{\prime} & j^{\prime} & l^{\prime}
\end{array}\right\}\right]\right] . }
\end{aligned}
$$

The definition of $s_{\alpha}^{\tau \nu}$ is given in (A3) of Appendix A. Furthermore, in (71) we have incorporated the phase shift for convenience into the quantity $\widetilde{N}_{\lambda}^{L}(\mu j)=e^{i \delta_{\mu}^{j}} N_{\lambda}^{L}(\mu j)$.

Then the general multipole decomposition reads

$$
f_{\alpha}^{(\prime) I M( \pm)}(X)=\sum_{K, \kappa \in \kappa X} f_{\alpha}^{(\prime) I M( \pm), K \kappa}(X) d_{-M-\beta(\alpha), \kappa}^{K}(\theta),
$$

where $\beta(\alpha)$ is listed in Table VIII and the coefficients $f_{\alpha}^{(\prime) I M( \pm), K \kappa}(X)$ are obtained via (49) from the foregoing multipole expansion. In detail one has for the longitudinal and transverse structure functions of an observable $X$

$$
\begin{aligned}
f_{L}^{I M, K \kappa}(X) & =\sum_{L^{\prime} \mu^{\prime} j^{\prime} L \mu j} \widetilde{\mathcal{C}}_{L}^{I M, K}\left(L^{\prime} j^{\prime} L j\right) \widetilde{\mathcal{D}}_{\alpha^{\prime} \alpha}^{K \kappa}\left(\mu^{\prime} j^{\prime} \mu j\right) \Re e\left(i^{\delta_{I}^{X}+\delta_{\left(\alpha^{\prime}, \alpha\right)}^{(2)}} \widetilde{C}^{L^{\prime} *}\left(\mu^{\prime} j^{\prime}\right) \widetilde{C}^{L}(\mu j)\right), \\
f_{T}^{I M, K \kappa}(X) & =\sum_{L^{\prime} \mu^{\prime} j^{\prime} L \mu j} \widetilde{\mathcal{C}}_{T}^{I M, K}\left(L^{\prime} j^{\prime} L j\right) \widetilde{\mathcal{D}}_{\alpha^{\prime} \alpha}^{K \kappa}\left(\mu^{\prime} j^{\prime} \mu j\right) \Re e\left(i^{\bar{\delta}_{I}^{X}+\delta_{\left(\alpha^{\prime}, \alpha\right)}^{(2)}} \widetilde{N}_{1}^{L^{\prime} *}\left(\mu^{\prime} j^{\prime}\right) \widetilde{N}_{1}^{L}(\mu j)\right), \\
f_{T}^{\prime I M, K \kappa}(X) & =-\sum_{L^{\prime} \mu^{\prime} j^{\prime} L \mu j} \widetilde{\mathcal{C}}_{T}^{I M, K}\left(L^{\prime} j^{\prime} L j\right) \widetilde{\mathcal{D}}_{\alpha^{\prime} \alpha}^{K \kappa}\left(\mu^{\prime} j^{\prime} \mu j\right) \Im m\left(i^{X}+\delta_{\left(\alpha^{\prime}, \alpha\right)}^{(2)} \widetilde{N}_{1}^{L^{\prime} *}\left(\mu^{\prime} j^{\prime}\right) \widetilde{N}_{1}^{L}(\mu j)\right),
\end{aligned}
$$

and for the interference ones, distinguishing observables of type $A$

$$
\begin{aligned}
& f_{T T}^{I M \pm, K \kappa}(X)=\sum_{L^{\prime} \mu^{\prime} j^{\prime} L \mu j} \widetilde{\mathcal{C}}_{T T}^{I M \pm, K}\left(L^{\prime} j^{\prime} L j\right) \widetilde{\mathcal{D}}_{\alpha^{\prime} \alpha}^{K \kappa}\left(\mu^{\prime} j^{\prime} \mu j\right) \Re e\left(i^{\bar{\delta}_{I}^{X}+\delta_{\left(\alpha^{\prime}, \alpha\right)}^{(2)}} \widetilde{N}_{-1}^{L^{\prime} *}\left(\mu^{\prime} j^{\prime}\right) \widetilde{N}_{1}^{L}(\mu j)\right), \\
& f_{L T}^{I M \pm, K \kappa}(X)=\sum_{L^{\prime} \mu^{\prime} j^{\prime} L \mu j} \widetilde{\mathcal{C}}_{L T}^{I M \pm, K}\left(L^{\prime} j^{\prime} L j\right) \widetilde{\mathcal{D}}_{\alpha^{\prime} \alpha}^{K \kappa}\left(\mu^{\prime} j^{\prime} \mu j\right) \Re e\left(i^{\bar{\delta}_{I}^{X}+\delta_{\left(\alpha^{\prime}, \alpha\right)}^{(2)}} \widetilde{C}^{L^{\prime} *}\left(\mu^{\prime} j^{\prime}\right) \widetilde{N}_{1}^{L}(\mu j)\right), \\
& f_{L T}^{\prime I M \pm, K \kappa}(X)=-\sum_{L^{\prime} \mu^{\prime} j^{\prime} L \mu j} \widetilde{\mathcal{C}}_{L T}^{I M \pm, K}\left(L^{\prime} j^{\prime} L j\right) \widetilde{\mathcal{D}}_{\alpha^{\prime} \alpha}^{K \kappa}\left(\mu^{\prime} j^{\prime} \mu j\right) \Im m\left(i^{\bar{\delta}_{I}^{X}+\delta_{\left(\alpha^{\prime}, \alpha\right)}^{(2)}} \widetilde{C}^{L^{\prime} *}\left(\mu^{\prime} j^{\prime}\right) \widetilde{N}_{1}^{L}(\mu j)\right),
\end{aligned}
$$

and observables of type $B$

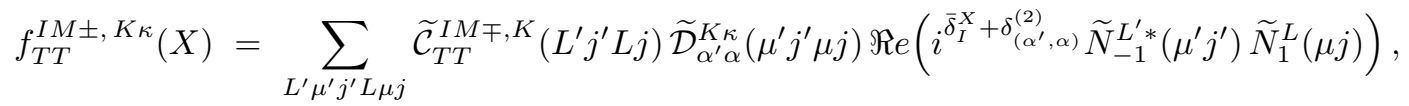

$$
\begin{aligned}
& f_{L T}^{I M \pm, K \kappa}(X)=\sum_{L^{\prime} \mu^{\prime} j^{\prime} L \mu j} \widetilde{\mathcal{C}}_{L T}^{I M \mp, K}\left(L^{\prime} j^{\prime} L j\right) \widetilde{\mathcal{D}}_{\alpha^{\prime} \alpha}^{K \kappa}\left(\mu^{\prime} j^{\prime} \mu j\right) \Re e\left(i^{\bar{\delta}_{I}^{X}+\delta_{\left(\alpha^{\prime}, \alpha\right)}^{(2)}} \widetilde{C}^{L^{\prime} *}\left(\mu^{\prime} j^{\prime}\right) \widetilde{N}_{1}^{L}(\mu j)\right), \\
& f_{L T}^{\prime I M \pm, K \kappa}(X)=-\sum_{L^{\prime} \mu^{\prime} j^{\prime} L \mu j} \widetilde{\mathcal{C}}_{L T}^{I M \mp, K}\left(L^{\prime} j^{\prime} L j\right) \widetilde{\mathcal{D}}_{\alpha^{\prime} \alpha}^{K \kappa}\left(\mu^{\prime} j^{\prime} \mu j\right) \Im m\left(i^{\bar{\delta}_{I}^{X}+\delta_{\left(\alpha^{\prime}, \alpha\right)}^{(2)}} \widetilde{C}^{L^{\prime} *}\left(\mu^{\prime} j^{\prime}\right) \widetilde{N}_{1}^{L}(\mu j)\right) .
\end{aligned}
$$


Here the coefficients $\widetilde{\mathcal{C}_{\alpha}}$ are defined by

$$
\begin{aligned}
\widetilde{\mathcal{C}}_{L}^{I M, K}\left(L^{\prime} j^{\prime} L j\right) & =\frac{8 \pi}{1+\delta_{M 0}} \mathcal{C}^{00 I M, K}\left(L^{\prime} j^{\prime} L j\right), \\
\widetilde{\mathcal{C}}_{T}^{I M, K}\left(L^{\prime} j^{\prime} L j\right) & =\frac{16 \pi}{1+\delta_{M 0}} \mathcal{C}^{11 I M, K}\left(L^{\prime} j^{\prime} L j\right), \\
\widetilde{\mathcal{C}}_{L T}^{I M \pm, K}\left(L^{\prime} j^{\prime} L j\right) & =\frac{16 \pi}{1+\delta_{M 0}}\left(\mathcal{C}^{01 I M, K}\left(L^{\prime} j^{\prime} L j\right) \pm(-)^{I+M} \mathcal{C}^{01 I-M, K}\left(L^{\prime} j^{\prime} L j\right)\right), \\
\widetilde{\mathcal{C}}_{T T}^{I M \pm, K}\left(L^{\prime} j^{\prime} L j\right) & =\frac{8 \pi}{1+\delta_{M 0}}\left(\mathcal{C}^{-11 I M, K}\left(L^{\prime} j^{\prime} L j\right) \pm(-)^{I+M} \mathcal{C}^{-11 I-M, K}\left(L^{\prime} j^{\prime} L j\right)\right) .
\end{aligned}
$$

More detailed expressions for the coefficients of the structure functions of the differential cross section are listed in the Appendix C. Explicit results for the coefficients $\widetilde{\mathcal{C}}$ and $\widetilde{\mathcal{D}}$ for a maximal multipolarity $L_{\max }=3$ may be found in $[10]$.

\section{F. Inclusive process and form factors}

The inclusive cross section is obtained by integration over the solid angle $\Omega_{n p}^{\text {c.m. }}=(\theta, \phi)$ yielding

$$
\begin{aligned}
\frac{d \sigma}{d k_{2}^{\mathrm{lab}} d \Omega_{e}^{\mathrm{lab}}}= & 6 c\left(k_{1}^{\mathrm{lab}}, k_{2}^{\mathrm{lab}}\right)\left\{\rho_{L} F_{L}+\rho_{T} F_{T}-P_{1}^{d} \rho_{L T} F_{L T}^{1-1} \sin \phi_{d} d_{10}^{1}\left(\theta_{d}\right)\right. \\
& +P_{2}^{d}\left[\left(\rho_{L} F_{L}^{20}+\rho_{T} F_{T}^{20}\right) d_{00}^{2}\left(\theta_{d}\right)-\rho_{L T} F_{L T}^{2-1} \cos \phi_{d} d_{10}^{2}\left(\theta_{d}\right)+\rho_{T T} F_{T T}^{2-2} \cos 2 \phi_{d} d_{20}^{2}\left(\theta_{d}\right)\right] \\
& \left.+h P_{1}^{d}\left[-\rho_{T}^{\prime} F_{T}^{\prime 10} d_{00}^{1}\left(\theta_{d}\right)+\rho_{L T}^{\prime} F_{L T}^{\prime 1-1} \cos \phi_{d} d_{10}^{1}\left(\theta_{d}\right)\right]-h P_{2}^{d} \rho_{L T}^{\prime} F_{L T}^{\prime 2-1} \sin \phi_{d} d_{10}^{2}\left(\theta_{d}\right)\right\} \\
\equiv & \sigma\left(h, P_{1}^{d}, P_{2}^{d}\right) .
\end{aligned}
$$

It is governed by a set of inclusive form factors $F_{\alpha}^{(\prime) I-M}(M \geq 0)$ as given by

$$
F_{\alpha}^{(\prime) I-M}=(-)^{I+M}\left(1+\delta_{M 0}\right) \frac{\pi}{6} \int d(\cos \theta)\left(f_{\alpha}^{(\prime) I M+}-f_{\alpha}^{(\prime) I M-}\right),
$$

for $\alpha \in\{L, T, L T, T T\}$. This equation corresponds to Eqs. (13) and (14) of [1] except for the fact that the primed form factors $F_{T}^{\prime 10}$ and $F_{L T}^{\prime 1-1}$ differ in sign from the ones given in [1] due to a redefinition of the primed structure functions incorporating a phase factor $(-)^{I}$ (see the remark in [4] before Eq. (9)). Altogether, the inclusive cross section depends on ten form factors: $F_{L}, F_{T}, F_{L T}^{1-1}, F_{L}^{20}, F_{T}^{20}, F_{L T}^{2-1}, F_{T T}^{2-2}, F_{T}^{\prime 10}, F_{L T}^{\prime 1-1}$, and $F_{L T}^{\prime 2-1}$, of which $F_{L T}^{1-1}$ and $F_{L T}^{\prime 2-1}$ vanish below pion threshold due to time reversal invariance.

The multipole decomposition of the inclusive form factors are given by the $(K=0)$-coefficients of the multipole expansion of the structure functions of the differential cross section $(X=1=(0,0))$, as listed in (73), i.e.

$$
F_{\alpha}^{(\prime) I-M}=(-)^{I+M}\left(1+\delta_{M 0}\right) \frac{\pi}{3}\left(f_{\alpha}^{(\prime) I M+, 0}-f_{\alpha}^{(\prime) I M-, 0}\right) .
$$

Explicit expressions are listed in Appendix C.

At the photon point one can relate the purely transverse form factors to the total photoabsorption cross section $\sigma_{t o t}$ of deuteron photodisintegration for unpolarized photons and deuterons and to the corresponding beam and target asymmetries of the total cross section as defined in [18]. Taking into account the relations (55) one obtains respectively

$$
\sigma_{\text {tot }}=\frac{M_{d}}{W_{n p} q^{c . m .}} F_{T}, \quad \tau_{20}^{0}=\frac{F_{T}^{20}}{F_{T}} \quad \tau_{10}^{c}=\frac{F_{T}^{\prime 10}}{F_{T}}, \quad \tau_{22}^{l}=\frac{F_{T T}^{2-2}}{F_{T}},
$$

where $W_{n p}$ and $q^{\text {c.m. }}$. denote the invariant mass of the $n p$ system and the photon c.m. momentum, respectively.

\section{SEPARATION OF STRUCTURE FUNCTIONS AND COMPLETE SETS}

\section{A. Experimental separation of structure functions}

The experimental separation of structure functions has been discussed in detail in $[2,4]$. It is based on the general definition of asymmetries of a polarization observable with respect to the beam and target polarization parameters $h$, 
$P_{1}^{d}$, and $P_{2}^{d}$, respectively. To this end one writes a general polarization observable $\mathcal{O}\left(\Omega_{X}\right)$ as given in (24) and (52) in the form

$$
P_{X}=A_{0}(X)+P_{1}^{d} A_{d}^{V}(X)+P_{2}^{d} A_{d}^{T}(X)+h\left[A_{e}(X)+P_{1}^{d} A_{e d}^{V}(X)+P_{2}^{d} A_{e d}^{T}(X)\right],
$$

defining implicitly the various asymmetries, i.e. $A_{e}(X)$ for beam polarization, $A_{d}^{V}(X)$ and $A_{d}^{T}(X)$ for vector and tensor target polarization, respectively, and $A_{e d}^{V}(X)$ and $A_{e d}^{T}(X)$ for the corresponding beam-target asymmetries. Their explicit form can be read from (52)

$$
\begin{aligned}
& A_{0}(X)=\frac{c\left(k_{1}^{\text {lab }}, k_{2}^{\text {lab }}\right)}{S_{0}}\left[\left(\rho_{L} f_{L}^{00}(X)+\rho_{T} f_{T}^{00}(X)+\rho_{L T} f_{L T}^{00+}(X) \cos \phi+\rho_{T T} f_{T T}^{00+}(X) \cos 2 \phi\right) \delta_{X, A}\right. \\
& \left.+\left(\rho_{L T} f_{L T}^{00-}(X) \sin \phi+\rho_{T T} f_{T T}^{00-}(X) \sin 2 \phi\right) \delta_{X, B}\right], \\
& A_{d}^{V}(X)=\frac{c\left(k_{1}^{\mathrm{lab}}, k_{2}^{\mathrm{lab}}\right)}{S_{0}} \sum_{M=0}^{1}\left[\left(\rho_{L} f_{L}^{1 M}(X)+\rho_{T} f_{T}^{1 M}(X)+\rho_{L T} f_{L T}^{1 M+}(X) \cos \phi\right.\right. \\
& \left.+\rho_{T T} f_{T T}^{1 M+}(X) \cos 2 \phi\right) \cos \left(M \tilde{\phi}-\delta_{X, A} \frac{\pi}{2}\right) \\
& \left.-\left(\rho_{L T} f_{L T}^{1 M-}(X) \sin \phi+\rho_{T T} f_{T T}^{1 M-}(X) \sin 2 \phi\right) \sin \left(M \tilde{\phi}-\delta_{X, A} \frac{\pi}{2}\right)\right] d_{M 0}^{1}\left(\theta_{d}\right), \\
& A_{d}^{T}(X)=\frac{c\left(k_{1}^{\mathrm{lab}}, k_{2}^{\mathrm{lab}}\right)}{S_{0}} \sum_{M=0}^{2}\left[\left(\rho_{L} f_{L}^{2 M}(X)+\rho_{T} f_{T}^{2 M}(X)+\rho_{L T} f_{L T}^{2 M+}(X) \cos \phi\right.\right. \\
& \left.+\rho_{T T} f_{T T}^{2 M+}(X) \cos 2 \phi\right) \cos \left(M \tilde{\phi}-\delta_{X, B} \frac{\pi}{2}\right) \\
& \left.-\left(\rho_{L T} f_{L T}^{2 M-}(X) \sin \phi+\rho_{T T} f_{T T}^{2 M-}(X) \sin 2 \phi\right) \sin \left(M \tilde{\phi}-\delta_{X, B} \frac{\pi}{2}\right)\right] d_{M 0}^{2}\left(\theta_{d}\right), \\
& A_{e}(X)=\frac{c\left(k_{1}^{\text {lab }}, k_{2}^{\text {lab }}\right)}{S_{0}}\left[-\left(\rho_{T}^{\prime} f_{T}^{\prime 00}(X)+\rho_{L T}^{\prime} f_{L T}^{\prime 00-}(X) \cos \phi\right) \delta_{X, B}+\rho_{L T}^{\prime} f_{L T}^{\prime 00+}(X) \sin \phi \delta_{X, A}\right] \text {, } \\
& A_{e d}^{V}(X)=\frac{c\left(k_{1}^{\text {lab }}, k_{2}^{\text {lab }}\right)}{S_{0}} \sum_{M=0}^{1}\left[\left(\rho_{T}^{\prime} f_{T}^{\prime 1 M}(X)+\rho_{L T}^{\prime} f_{L T}^{\prime 1 M-}(X) \cos \phi\right) \sin \left(M \tilde{\phi}-\delta_{X, A} \frac{\pi}{2}\right)\right. \\
& \left.+\rho_{L T}^{\prime} f_{L T}^{\prime 1 M+}(X) \sin \phi \cos \left(M \tilde{\phi}-\delta_{X, A} \frac{\pi}{2}\right)\right] d_{M 0}^{1}\left(\theta_{d}\right), \\
& A_{e d}^{T}(X)=\frac{c\left(k_{1}^{\text {lab }}, k_{2}^{\text {lab }}\right)}{S_{0}} \sum_{M=0}^{2}\left[\left(\rho_{T}^{\prime} f_{T}^{\prime 2 M}(X)+\rho_{L T}^{\prime} f_{L T}^{\prime 2 M-}(X) \cos \phi\right) \sin \left(M \tilde{\phi}-\delta_{X, B} \frac{\pi}{2}\right)\right. \\
& \left.+\rho_{L T}^{\prime} f_{L T}^{\prime 2 M+}(X) \sin \phi \cos \left(M \tilde{\phi}-\delta_{X, B} \frac{\pi}{2}\right)\right] d_{M 0}^{2}\left(\theta_{d}\right),
\end{aligned}
$$

where the unpolarized differential cross section $S_{0}$ is defined in (53). For simplicity, we will also call $A_{0}(X)$ an asymmetry although it is not one in the strict sense. The nonvanishing structure functions contributing to an asymmetry of a given observable are listed in Table II. For the differential cross section $(X=1=(00))$ we remind the reader that one has with respect to the notation in $[2,40]$

$$
A_{0}(1)=1, \quad A_{d}^{V / T}(1)=A_{d}^{V / T}, \quad A_{e}(1)=A_{e}, \quad A_{e d}^{V / T}(1)=A_{e d}^{V / T} .
$$

For the simplest case, namely in the absence of beam and target polarization, the four structure functions $f_{\alpha}(X)$ can be separated choosing first different $\phi$-angles, yielding $f_{L T}, f_{T T}$ and a linear superposition of $f_{L}$ and $f_{T}$ and subsequently a RosEnBLUTH separation for disentangling $f_{L}$ and $f_{T}$. In the general case, by a proper variation of the longitudinal electron polarization $h$ and the deuteron vector and tensor polarization parameters $P_{1}^{d}$ and $P_{2}^{d}$, respectively, one can first separate the various beam, target and beam-target asymmetries as listed in (83). These asymmetries are functions of the deuteron orientation angles $\theta_{d}$ and $\phi_{d}$, viz. $\tilde{\phi}=\phi-\phi_{d}$, and the azimuthal or outof-plane angle $\phi$. One can now utilize these variables for the further separation of the different structure functions.

This is achieved by observing that the general functional form of an asymmetry is

$$
A^{I}\left(\phi, \tilde{\phi}, \theta_{d}\right)=\sum_{M=0}^{I} \alpha_{I M}(\phi, \tilde{\phi}) d_{M 0}^{I}\left(\theta_{d}\right), \quad(I=0,1,2),
$$


where

$$
\alpha_{I M}(\phi, \tilde{\phi})=c_{I M}(\phi) \cos M \tilde{\phi}+s_{I M}(\phi) \sin M \tilde{\phi},
$$

and the $\phi$-dependent functions $c_{I M}(\phi)$ and $s_{I M}(\phi)$ have either the form

$$
a_{0}+a_{1} \cos \phi+a_{2} \cos 2 \phi
$$

or

$$
b_{1} \sin \phi+b_{2} \sin 2 \phi .
$$

For a given $I$ the $M$-components $\alpha_{I M}(\phi, \tilde{\phi})$ of the asymmetry $A^{I}\left(\phi, \tilde{\phi}, \theta_{d}\right)$ can be separated by a proper choice of $\theta_{d}$ exploiting the properties of the small $d_{M 0}^{I}$-functions. For $I=1$ (vector asymmetries), taking $\theta_{d}=0$ or $\pi / 2$, i.e. $d_{M 0}^{1}(0)=\delta_{M 0}$ or $d_{M 0}^{1}(\pi / 2)=M / \sqrt{2}$, yields $\alpha_{10}$ or $\alpha_{11}$, respectively, and for the tensor asymmetries $(I=2)$ one may first choose $\theta_{d}=0$ yielding with $d_{M 0}^{2}(0)=\delta_{M 0}$ directly $\alpha_{20}$. The latter being determined, then setting $\theta_{d}=\pi / 4$ and $\pi / 2$, one can obtain the remaining two terms $\alpha_{21}$ and $\alpha_{22}$. For the separation of $\alpha_{21}$ and $\alpha_{22}$ one can also choose $\theta_{d}=\theta_{d}^{0}=\operatorname{arcos}(1 / \sqrt{3})$ together with $\tilde{\phi}$ and $\tilde{\phi}+\pi$. Then the sum and difference of the corresponding asymmetries result in $\alpha_{21}$ and $\alpha_{22}$, respectively.

In the next step, in order to separate the two contributions $c_{I M}$ and $s_{I M}$ in (86), one can take first $\tilde{\phi}=0$ giving $c_{I M}$ and then $\tilde{\phi}=\pi / 2 M$ for $M \neq 0$ which yields directly $s_{I M}$. The remaining separation of the coefficients $a_{n}$ or $b_{n}$ in (87) is then achieved by appropriate choices of $\phi$. In a few cases the constant term $a_{0}$ in (87a) will contain two structure functions in the combination $\rho_{L} f_{L}^{I M}(X)+\rho_{T} f_{T}^{I M}(X)$. In this case one needs a Rosenbluth separation in addition.

A different task than the complete separation of all structure functions is to find an optimal way for separating a specific structure function $f_{\alpha}^{I M( \pm)}(X)(I>0)$ or $f_{\alpha}^{\prime I M( \pm)}(X)$. In other words, the question is: what is the minimal number of measurements necessary for the separation of a specific structure function? This has been discussed in [4] and is described in detail in Appendix D.

\section{B. Complete sets of observables}

We have already mentioned the fact that in deuteron electrodisintegration the total number of independent complex $t$-matrix elements is 18 , while for photodisintegration the number is 12 . Since one phase remains arbitrary this means that this process is determined by 35 independent observables, whereas in the corresponding photoreaction one needs 23. The question is how to choose from the much larger set of 324 (or 144 in photodisintegration) linearly independent observables an appropriate set of 35 (or 23). In [5] we had derived a general criterion which allows one to decide uniquely whether for a reaction with $n$ independent $t$-matrix elements a set of $2 n-1$ observables, taken from the set of $n^{2}$ linearly independent observables, constitutes a complete set. Subsequently this criterion has been applied to deuteron electro- and photodisintegration in [6]. A brief review of the main results of [5] and [6] is appropriate.

\section{General criterion [5]}

Any observable in a reaction with $n$ independent, complex matrix elements can be represented by a $n \times n$ hermitean form $f^{\alpha}$ in the complex $n$-dimensional variable $z=\left(z_{1}, \ldots, z_{n}\right)$

$$
f^{\alpha}(z)=\frac{1}{2} \sum_{j^{\prime} j} z_{j^{\prime}}^{*} F_{j^{\prime} j}^{\alpha} z_{j},
$$

where hermiticity requires

$$
\left(F_{j^{\prime} j}^{\alpha}\right)^{*}=F_{j j^{\prime}}^{\alpha},
$$

and $z$ comprises all independent reaction matrix elements labeled by $j$.

For the application of our criterion, derived in [5], one first has to rewrite the hermitean form in (88) into a real quadratic form by introducing

$$
\begin{aligned}
z & =x+i y, \\
F^{\alpha} & =A^{\alpha}+i B^{\alpha},
\end{aligned}
$$


where $A^{\alpha}$ and $B^{\alpha}$ are real matrices, and $A^{\alpha}$ is symmetric whereas $B^{\alpha}$ is antisymmetric. Considering further the fact that one overall phase is arbitrary, one may choose $y_{j_{0}}=0$ for an arbitrary index $j_{0}$ and then one finds for the given observable

$$
f^{\alpha}(x+i y)=\frac{1}{2}\left[\sum_{j^{\prime} j} x_{j^{\prime}} A_{j^{\prime} j}^{\alpha} x_{j}+\sum_{\tilde{j}^{\prime} \tilde{j}} y_{j^{\prime}} A_{j^{\prime} j}^{\alpha} y_{j}+2 \sum_{\tilde{j}^{\prime} j} y_{j^{\prime}} B_{j^{\prime} j}^{\alpha} x_{j}\right],
$$

where the tilde over a summation index indicates that the index $j_{0}$ has to be left out. Introducing now a $(m=2 n-1)$ dimensional real vector $u$ by

$$
u=\left(x_{1}, \ldots, x_{n}, y_{1}, \ldots, y_{j_{0}-1}, y_{j_{0}+1}, \ldots, y_{n}\right),
$$

one can represent the $n \times n$ hermitean form by a $m \times m$ real quadratic form

$$
\tilde{f}^{\alpha}(u)=\frac{1}{2} \sum_{l^{\prime} l=1}^{m} u_{l^{\prime}} \widetilde{F}_{l^{\prime} l}^{\alpha} u_{l},
$$

where the $m \times m$-matrix $\widetilde{F}^{\alpha}$ is given by

$$
\widetilde{F}^{\alpha}=\left(\begin{array}{cc}
A^{\alpha} & \left(\widetilde{B}^{\alpha}\right)^{T} \\
\widetilde{B}^{\alpha} & \widehat{A}^{\alpha}
\end{array}\right)
$$

Here $\widetilde{B}^{\alpha}$ is obtained from $B^{\alpha}$ by canceling the $j_{0}$-th row, and $\widehat{A}^{\alpha}$ from $A^{\alpha}$ by canceling the $j_{0}$-th row and column. Thus $\widetilde{B}^{\alpha}$ is a $(n-1) \times n$-matrix and $\widehat{A}^{\alpha}$ a $(n-1) \times(n-1)$-matrix.

Now, for checking the completeness of a chosen set of $2 n-1$ observables one has to construct the $m \times m$ corresponding matrices $\widetilde{F}^{\alpha}$, and then one builds from their columns for all possible sets $\left\{k_{1}, \ldots, k_{m} ; k_{\alpha} \in\{1, \ldots, m\}\right\}$ the matrices

$$
\widetilde{W}\left(k_{1}, \ldots, k_{m}\right)=\left(\begin{array}{ccc}
\widetilde{F}_{1 k_{1}}^{1} & \cdots & \widetilde{F}_{1 k_{m}}^{m} \\
\vdots & & \vdots \\
\widetilde{F}_{m k_{1}}^{1} & \cdots & \widetilde{F}_{m k_{m}}^{m}
\end{array}\right)
$$

Note that the $k_{\alpha}$ need not be different. If at least one of the determinants of $\widetilde{W}\left(k_{1}, \ldots, k_{m}\right)$ is nonvanishing then one has a complete set.

\section{Complete sets for photo- and electrodisintegration [6]}

In order to apply our criterion, one has to construct the matrices $\widetilde{F}$ which represent the structure functions as hermitean forms in the reaction matrix elements as

$$
f_{\alpha}^{(\prime) I M \pm}(X)=t^{\dagger} \widetilde{F}^{(\prime) I M \pm, \alpha} t
$$

where $t$ is a vector comprising all reduced $t$-matrix elements in a certain labeling. It is convenient to arrange the labeling of the $t$-matrix elements in such a way that the longitudinal ones belong to $j=1, \ldots, 6$ and the transverse ones to $j=7, \ldots, 18$. Thus the general structure of these matrices then is

$$
\widetilde{F}^{(\prime) I M \pm, \alpha}=\left(\begin{array}{cc}
A^{(\prime) I M \pm, \alpha} & C^{(\prime) I M \pm, \alpha} \\
\left(C^{(\prime) I M \pm, \alpha}\right)^{\dagger} & B^{(\prime) I M \pm, \alpha}
\end{array}\right)
$$

where $A^{(\prime) I M \pm, \alpha}$ is a $(6 \times 6)$-matrix, $C^{(\prime) I M \pm, \alpha}$ a $(6 \times 12)$-matrix, and $B^{(\prime) I M \pm, \alpha}$ a $(12 \times 12)$-matrix. In particular one has

$$
\begin{aligned}
& \widetilde{F}^{I M \pm, L}=\left(\begin{array}{cc}
A^{I M \pm, L} & 0 \\
0 & 0
\end{array}\right), \\
& \widetilde{F}^{(\prime) I M \pm, T / T T}=\left(\begin{array}{cc}
0 & 0 \\
0 & B^{(\prime) I M \pm, T / T T}
\end{array}\right), \\
& \widetilde{F}^{(\prime) I M \pm, L T}=\left(\begin{array}{cc}
0 & C^{(\prime) I M \pm, L T} \\
\left(C^{(\prime) I M \pm, L T}\right)^{\dagger} & 0
\end{array}\right) .
\end{aligned}
$$


The explicit forms of these matrices are obtained from the matrix representation of

$$
\mathcal{U}_{X}^{\lambda^{\prime} \lambda I M}=\sum_{j^{\prime} j} t_{j^{\prime}}^{*} \widetilde{C}_{j^{\prime} j}^{I M \lambda^{\prime} \lambda}(X) t_{j}
$$

Comparison with (51) gives for the matrix elements

$$
\widetilde{C}_{j^{\prime} j}^{I M \lambda^{\prime} \lambda}(X)=\left\langle m_{1}^{\prime} m_{2}^{\prime}\left|\sigma_{\alpha^{\prime}}(p) \sigma_{\alpha}(n)\right| m_{1} m_{2}\right\rangle\left\langle\lambda_{d}\left|\tau_{M}^{[I]}\right| \lambda_{d}^{\prime}\right\rangle,
$$

where the labeling is to be understood as $j^{(\prime)}=\left(m_{1}^{(\prime)}, m_{2}^{(\prime)}, \lambda^{(\prime)}, \lambda_{d}^{(\prime)}\right)$. Detailed expressions of the $\widetilde{C}^{I M \lambda^{\prime} \lambda}(X)$ 's for several representations are easily obtained from the expressions listed in Appendix E (see also [6]).

The structure of these matrices is such that the longitudinal $(L)$ and the transverse $(T, T T)$ observables are decoupled filling separated $6 \times 6$ - and $12 \times 12$-submatrices, respectively, whereas the $L T$-type observables are represented by $18 \times 18$-matrices. These features offer various kinds of strategies for selecting complete sets.

(i) One may independently select complete sets of observables for the longitudinal and transverse cases, i.e., a set of 11 longitudinal and 23 transverse structure functions for a check of completeness. With respect to the latter, one has in view of the linear relations between the $T$ - and the $T T^{+}$-type and between the $T^{\prime}$ - and $T T^{-}$-type observables, different choices, taking either $T$ - and $T^{\prime}$-type or $T T^{ \pm}$-type observables or even mixing different types of observables. The missing relative phase between the longitudinal and transverse $t$-matrix elements can then be provided by any one of the $L T$-observables. The advantage of this approach is that in this way one automatically obtains complete sets of observables for the case of photodisintegration as well, namely from the transverse ones.

(ii) Again one may start with a selection of 11 longitudinal structure functions. But then instead of choosing transverse observables, one may directly choose 24 linearly independent $L T$-type observables which then constitute a simple system of linear equations for the missing transverse matrix elements, because the longitudinal ones are then known from the first step.

(iii) Complementary to case (ii) one may start with a selection of 23 transverse observables taking one of the alternatives listed in (i). Then a proper set of $12 L T$-type observables provides a set of linear equations from which the missing longitudinal $t$-matrix elements can be obtained.

(iv) Another alternative would be a selection of 35 structure functions of $L T$-type. However, in this case the completeness check would be much more involved due to the considerably higher dimension of the determinants to be checked.

Which of these strategies is most advantageous will depend on the experimental conditions. Often $L$ - and $T$-type structure functions are easier to determine in an experiment although the required RoSENBLUTH separation introduces some unwanted complication. In view of the fact that the strategies (i) through (iii) require the determination of either $L$ - or $T$-type observables or both, we have considered in $[5,6]$ exclusively the question of complete sets for longitudinal and transverse structure functions.

The choice of complete sets of longitudinal structure functions, containing eleven structure functions, from a set of linearly independent ones has been discussed in detail in [5]. It turned out, that there is only a very weak restriction on the choice of possible complete longitudinal sets. In fact one may select from the chosen set of 36 linearly independent observables any subset of eleven structure functions, which does not contain more than eight of the type $X^{10}$ and $X^{22}$. This has been discussed explicitly for the linearly independent set $X \in\left\{1^{I M}, x x^{I M}, x z^{I M}, y_{1}^{I M}, x_{1}^{I M}, x_{2}^{I M}, z_{1}^{I M}, z_{2}^{I M}\right\}$, and possible complete sets are listed in Tables 3 and 4 of [5]. The case of the transverse observables for which a complete set contains 23 structure functions, has been discussed in [6]. Again with respect to the general question of a choice of a complete set of structure functions, we found the general statement, that one may pick from the chosen set of 144 linearly independent ones any subset of 23 structure functions with the only restriction, that not more than 16 should be of the type $X^{(\prime) 10}$ and $X^{(\prime) 22}$.

In Ref. [5] we furthermore simulated an experimental study for the determination of the longitudinal $t$-matrix elements in the helicity basis from a given set of "measured" observables whose numerical values were taken from a calculation. Various complete sets were selected and the arising system of 11 nonlinear equations for the $t$-matrix elements was solved numerically. Since the solutions were not unique we had to calculate additional observables, called "check observables", taking as input the obtained solutions for the $t$-matrix elements, and compared them to their "measured" values. For the arbitrarily chosen kinematics (internal excitation energy $E_{n p}=100 \mathrm{MeV}$, momentum transfer $\left(q^{\text {c.m. }}\right)^{2}=5 \mathrm{fm}^{-2}$, various $n p$-angles $\left.\theta\right)$ we found that one of the considered complete sets was particularly suitable (first set of Table 6 in Ref. [5]). In this case only one additional check observable $\left(f_{L}^{10}\left(x_{2}\right)\right)$ was sufficient 
to determine the correct solution. In [6] this simulation was extended to a somewhat more realistic experimental situation using the same kinematics again and taking the same specific set but allowing for errors in the measured observables. The simulation of an experimental situation showed that one can get quite reliable results for the $t$-matrix elements even if experimental errors are taken into account. The results can be greatly improved if additional check observables are considered. For the case studied in [6], it was sufficient to consider two such observables. If on the other hand one uses no check observables at all, one gets rather unreliable results since other types of solutions of the nonlinear system of equations are mixed in. In fact, performing such a simulation without any check observable leads to large errors in the resulting $t$-matrix elements (average error more than $100 \%$ ) and also to strong average deviations of the mean values from the true values of the $t$-matrix elements (about $50 \%$ ).

A similar study has been performed in [6] for the determination of the transverse $t$-matrix elements from observables, but without introducing experimental errors. Although the transverse case is much more complicated than the longitudinal one due to the higher dimensionality (12 instead of 6 complex $t$-matrix elements), it was found that in principle the method works also for the transverse case.

\section{Analytic expressions of the $t$-matrix elements in terms of observables}

One can also derive an analytic solution of the reaction matrix elements in terms of observables, because one can express all bilinear forms $t_{j^{\prime}}^{*} t_{j}$ as linear forms in the structure functions $f_{\alpha}^{(\prime) I M \pm}(X)$, i.e.,

$$
t_{j^{\prime}}^{*} t_{j}=T_{j^{\prime} j}\left[f_{\alpha}^{(\prime) I M \pm}(X)\right]=\sum_{\alpha I M, s i g= \pm}\left(T_{j^{\prime} j}^{\alpha I M s i g} f_{\alpha}^{I M s i g}(X)+T_{j^{\prime} j}^{\prime \alpha I M s i g} f_{\alpha}^{\prime I M s i g}(X)\right),
$$

where the explicitly appearing structure functions $f_{\alpha}^{(\prime) I M \pm}(X)$ constitute a complete, linearly independent set. Here the square bracket of $T_{j^{\prime} j}[f]$ indicates the functional dependence on the structure functions. This relation has also been used in the derivation of the linear relations between observables (see Sect. IID). Explicit expressions for the coefficients $T_{j^{\prime} j}^{(\prime) \alpha I M s i g}$ are derived in various representations in [6]. To give an example, we list here two cases, a diagonal and an interference term, of the longitudinal $t$-matrix elements in a rotated helicity basis, where the final helicity states are rotated into the $y$-axis,

$$
\begin{aligned}
t_{\frac{1}{2} \frac{1}{2} 00}^{*} t_{\frac{1}{2} \frac{1}{2} 00} & =\frac{1}{6}\left(f_{L}^{00}-\sqrt{2} f_{L}^{20}+f_{L}^{00}\left(y_{1}\right)-\sqrt{2} f_{L}^{20}\left(y_{1}\right)\right), \\
t_{-\frac{1}{2}-\frac{1}{2} 00}^{*} t_{\frac{1}{2} \frac{1}{2} 00} & =\frac{1}{6}\left(-f_{L}^{00}(z z)+\sqrt{2} f_{L}^{20}(z z)+i\left(f_{L}^{00}(x z)-\sqrt{2} f_{L}^{20}(x z)\right)\right) .
\end{aligned}
$$

For a complete listing and further details we refer to the Appendix E of [6].

The linear relations in (102) can be exploited in various ways. One possibility is to choose a specific matrix element, say $t_{j_{0}}$, as real and positive. Then all other matrix elements $t_{j}$ with $j \neq j_{0}$ are uniquely determined relative to $t_{j_{0}}$ and are given as linear forms of appropriate structure functions [8]

$$
t_{j}=\frac{1}{t_{j_{0}}} T_{j_{0} j}\left[f_{\alpha}^{(\prime) I M \pm}(X)\right] .
$$

Finally, for the determination of the missing matrix element $t_{j_{0}}$ one has to choose only one additional structure function, say

$$
f_{0}=\sum_{j^{\prime} j} t_{j^{\prime}}^{*} \tilde{F}_{j^{\prime} j} t_{j}
$$

yielding

$$
t_{j_{0}}=\frac{1}{\sqrt{f_{0}}} \sqrt{\sum_{j^{\prime} j} T_{j^{\prime} j_{0}}\left[f_{\alpha}^{(\prime) I M \pm}(X)\right] \tilde{F}_{j^{\prime} j} T_{j_{0} j}\left[f_{\alpha}^{(\prime) I M \pm}(X)\right]} .
$$

Thus (104) in conjunction with (106) constitutes a nonlinear functional in the structure functions $f_{\alpha}^{(\prime) I M \pm}(X)$. However, proceeding in this way, one needs in general a much larger number of observables for the complete determination of the $t$-matrix than the required minimal number of $2 n-1$ of a complete set of a $n$-dimensional $t$-matrix.

Another strategy, which leads in general to a smaller number of necessary observables, has been developed in [6]. It is based on an analysis of all interference terms with respect to the question, which and how many observables appear 

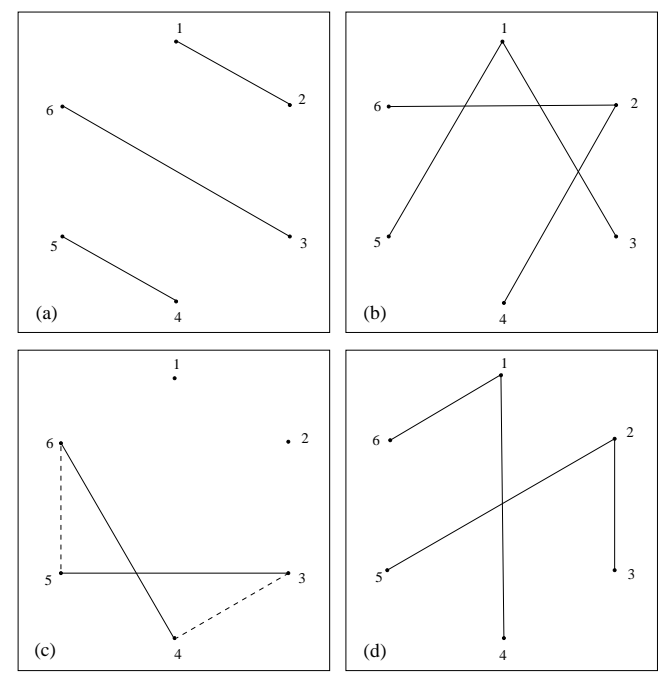

FIG. 2: Diagrammatic representation of groups of longitudinal observables determining the interference terms of $t$-matrix elements for the helicity basis. The nomenclature for the groups and the corresponding observables are listed in Table IX.

TABLE IX: Nomenclature for the diagrammatic representation of groups of longitudinal observables in Fig. 2 determining the interference terms of $t$-matrix elements for the helicity basis, where $f_{L}^{I M}(X)$ is represented by $X^{I M}$.

\begin{tabular}{lcl}
\hline \hline Panel & Line type & Observables \\
\hline (a) & solid & $x_{1}^{10}, x_{2}^{22}, y_{1}^{00}, y_{1}^{20}, x z^{00}, x z^{20}$ \\
(b) & solid & $1^{11}, 1^{21}, z_{1}^{11}, z_{2}^{11}, z_{1}^{21}, z_{2}^{21}, z z^{11}, z z^{21}$ \\
(c) & solid & $1^{22}, z_{1}^{22}, z_{2}^{22}, z z^{22}$ \\
& dashed & $x_{2}^{10}, x_{1}^{22}, y_{1}^{22}, x z^{22}$ \\
(d) & solid & $x_{1}^{11}, x_{2}^{11}, x_{1}^{21}, x_{2}^{21}, y_{1}^{11}, y_{1}^{21}, x z^{11}, x z^{21}$ \\
\hline \hline
\end{tabular}

in the representation of an interference term by observables. Because a closer inspection of the explicit expressions reveals, that in general the interference terms can be divided into disjunct subgroups which are determined by a subgroup of observables. In order to visualize this grouping we have devised in [6] a graphical representation. To this end one assembles the numbers " 1 " through " $n$ ", where $n$ denotes the number of $t$-matrix elements, by points on a circle and represents an interference term $t_{j^{\prime}}^{*} t_{j}$ by a straight line joining the points " $j$ " and " $j$ ". Interference terms belonging to the same subgroup are then represented by the same type of lines. An example for the longitudinal matrix elements in the helicity basis is shown in Fig. 2.

We will call a set of interference terms connected if they generate a pattern of connected lines so that any point belonging to one of the considered interference terms is connected to any other point of the set either directly or via $k$ other intermediate points of that set. For example, in Fig. 2(a) one notes three disconnected lines, (b) and (d) contain two different groups of connected lines, whereas (c) contains one connected group of four lines. In such a connected set, any matrix element $t_{j^{\prime}}$ can be expressed in terms of any other matrix element $t_{j}$ of that set by one of the two forms

$$
t_{j^{\prime}}= \begin{cases}\frac{T_{j_{1} j^{\prime}}}{T_{j_{1} j_{2}}} \frac{T_{j_{3} j_{2}}}{T_{j_{3} j_{4}}} \cdots \frac{T_{j_{k-2} j_{k-3}}}{T_{j_{j_{1}} j^{\prime}}} \frac{T_{j_{k} j_{k-1}}}{T_{j_{3} j_{2}}} t_{j} \quad \text { for } k \text { odd } \\ T_{j_{1} j_{2}} \frac{T_{j_{k-3} j_{k-4}}}{T_{j_{3} j_{4}}} \cdots \frac{T_{j_{k-1} j_{k-2}}}{T_{j_{k-3} j_{k-2}}} \frac{T_{j_{k}}}{T_{j_{k-1} j_{k}}} \frac{\text { for } k \text { even }}{t_{j}^{*}}\end{cases}
$$

depending on whether the number $k$ of intermediate points connecting $j^{\prime}$ with $j$ via the points $j_{1}$ through $j_{k}$ is odd or even. The proof of these equations is simple and given in [6].

A special feature of the evolving geometric pattern is a closed loop, see e.g. Fig. 2(c). If such a closed loop has an even number of points then one finds from (107) for $j^{\prime}=j$ and $k$ odd the following condition

$$
T_{j_{k} j_{k-1}} T_{j_{k-2} j_{k-3}} \cdots T_{j_{3} j_{2}} T_{j_{1} j}=T_{j_{1} j_{2}} T_{j_{3} j_{4}} \cdots T_{j_{k-2} j_{k-1}} T_{j_{k} j}
$$

which means that in such a closed loop any interference term is completely determined by the other remaining interference terms of that loop. This condition thus constitutes a complex relation between the participating observables 
which allows one to eliminate two observables. On the other hand, a closed loop through an odd number of points (107) yields for $j^{\prime}=j$ and $k$ even

$$
T_{j j_{k}} T_{j_{k-1} j_{k-2}} \cdots T_{j_{3} j_{2}} T_{j_{1 j}}=T_{j j} T_{j_{1} j_{2}} T_{j_{3} j_{4}} \cdots T_{j_{k-3} j_{k-2}} T_{j_{k-1} j_{k}},
$$

which again allows the elimination of two observables. It means furthermore that the modulus of each of the participating $t$-matrix elements is completely determined, namely one has

$$
\left|t_{j}\right|^{2}=\frac{T_{j_{1} j}}{T_{j_{1} j_{2}}} \frac{T_{j_{3} j_{2}}}{T_{j_{3} j_{4}}} \cdots \frac{T_{j_{k-3} j_{k-4}}}{T_{j_{k-3} j_{k-2}}} \frac{T_{j_{k-1} j_{k-2}}}{T_{j_{k-1} j_{k}}} T_{j j_{k}} .
$$

Thus one may choose one matrix element of that loop as real and non-negative, fix its modulus according to (110) and then all other matrix elements of that loop are uniquely determined. As a side remark, we would like to point out, that the conditions in (108) and (109) constitute particular nonlinear relations between observables, which follow from the ones discussed in the Appendix B. In fact, these conditions are obtained by applying successively the condition in (B4) of this appendix to the left-hand sides of (108) and (109) yielding then the corresponding right-hand sides.

As next step one has to choose from the total number of all interference terms $t_{j^{\prime}}^{*} t_{j}$ with $j^{\prime}>j$ which is $\frac{1}{2} n(n-1)$ - not counting $t_{j^{\prime}}^{*} t_{j}$ with $j^{\prime}<j$, because $\left(t_{j^{\prime}}^{*} t_{j}\right)^{*}=t_{j}^{*} t_{j^{\prime}}$ - a set of $n-1$ independent interference terms. Hereby we define a set of independent interference terms by the property that they generate a geometric pattern which does not contain any closed loop. From this definition follows that a set of $n-1$ independent interference terms is represented by a pattern of $n-1$ lines in such a fashion that (i) each of the $n$ points is endpoint of at least one line, and (ii) each point is connected to all other points not necessarily in a direct manner but via intermediate points. It is obvious that in such a pattern no closed loops can be present, because one cannot construct from $n-1$ lines a pattern which contains a closed loop and which still connects all $n$ points. For such a set of $n-1$ independent interference terms all matrix elements can be expressed by one arbitrarily chosen matrix element, say $t_{j_{0}}$ according to (107). In order to fix the remaining undetermined matrix element $t_{j_{0}}$ one has to choose one additional observable $f_{0}$. From (105) one obtains in general an equation of the type

$$
f_{0}=a+b\left|t_{j_{0}}\right|^{2}+c\left|t_{j_{0}}\right|^{-2},
$$

from which $t_{j_{0}}$ can be obtained, although not uniquely in general. The ideal situation would be such that one finds $n-1$ independent interference terms each of them represented by only two observables. Because in this case one employs just $2 n-1$ observables. On the other hand, analyzing the grouping of observables mentioned above, one will in general not find such a situation, either the number of observables for a set of $n-1$ independent interference terms is larger than $2 n-2$, or the grouping is such, that the choice of $n-1$ independent interference terms involves observables which govern at least one additional interference term leading to one or several closed loops. However, in that case those loops lead to the elimination of superfluous observables. For example, considering Fig. 2, one notes that combination of the three lines of (a) with two connected lines of the connected groups in (b) or (d) results in a set of independent interference terms. According to Table IX, such a choice involves 14 structure functions, which means, that all six longitudinal matrix elements can be expressed in terms of 15 observables. In order to eliminate four of them, one can include the other group of two connected lines of (c) or (d), yielding two closed loops which allow the elimination. Thus, at the end one has a complete set of eleven observables. Further illustrative examples for such an analysis are discussed in some detail in [6]. 


\section{INGREDIENTS OF CALCULATION}

In this section we will review briefly the various ingredients which go into the calculation of the structure functions presented in this work. The structure functions are determined by hermitean quadratic forms in the matrix elements of the e.m. current operator between the initial and final states. As already mentioned in Sect. II, the calculation is done with respect to the c.m. frame of the final hadronic $N N$ state. Thus for a momentum transfer $\vec{q}$ the initial deuteron moves in this reference frame with a momentum $-\vec{q}$, and one has to take into account the transformation to the lab frame as governed by the $\beta$-factor of (37).

Structure functions are calculated within a nonrelativistic framework from the $t$-matrix defined in (27). Therefore, the wave functions are purely nonrelativistic and are obtained by solving the two-body ScHRöDINGER equation with a realistic $N N$-potential for the bound as well as for the scattering states. Thus one of the principal ingredients of our calculation is a realistic potential model in order to generate the bound and scattering $n p$-wave functions.

The leading current contribution is provided by the nonrelativistic one-body nucleon current. As another important ingredient, we consider subnuclear degrees of freedom (d.o.f.) related to meson exchange currents (MEC) and isobar configurations (IC). Because of the increasing importance of relativistic effects with increasing energy and momentum transfer, we include also relativistic contributions of leading order beyond the nonrelativistic current.

In view of the fact that some realistic NN-potentials are defined in $r$-space while others are in $p$-space, we have employed two separate corresponding codes. The $r$-space code is an outgrowth of the work of FABIAN and ARENHÖVEL [23] incorporating improvements and additions, particularly with respect to the most important relativistic spin-orbit current and other leading order relativistic contributions. In this code, isobar configurations are treated in a perturbative approach [24] even though we had developed in the past a coupled channel $r$-space code [25]. However, in that code the $\Delta$-propagator could not be treated as exactly as in a $p$-space code.

The $p$-space code is described in detail in [26]. Besides inclusion of the $N \Delta$-configuration as the most important isobar configuration in a coupled channel approach, it allows furthermore an exact treatment of all leading order relativistic contributions to one- and two-body currents as well as the Lorentz boost for a one-boson-exchange $N N$ interaction model. In fact, this code was developed for the BonN $p$-space potentials (OBEPQ models) of [27] which are potentials of this type with short range cut-off form factors.

A further difference between the two codes lies in their treatment of the electric multipoles. The $r$-space version incorporates the SIEGERT operators thereby insuring the inclusion of the dominant MEC implicitly in electric transitions. In the $p$-space version, mainly for historical reasons, the SIEGERT form of the electric multipoles is not used. In view of the fact that some potentials, e.g. the BonN $r$-space, are derived from their corresponding $p$-space versions, one can estimate the inherent numerical differences between the two codes by using in this case the same potential model in both codes.

The calculation of the $t$-matrix elements is based on an expansion of the final state into partial waves with total angular momentum $j$. The final state interaction (FSI) is taken into account by solving the corresponding scattering equation for a given partial wave $\left|\vec{p}, j m_{j}\right\rangle$. Then the electric and magnetic multipole transitions into this state are evaluated explicitly. For a given $j$ the contributing multipoles are $L=j-1, j, j+1$. However, at some point one has to truncate the series at a maximal angular momentum $j_{\max }$ for the explicit inclusion of FSI. On the other hand, the convergence of the partial wave expansion depends on the kinematics, in fact the convergence is quite slow for the quasi-free case. The solution to this dilemma is based on the fact that for higher partial waves the influence of FSI becomes increasingly unimportant with growing $j$ so that for these the undistorted partial waves can be used instead. Thus we include all electric and magnetic multipoles up to a maximal multipolarity $L_{\max }$ with consideration of FSI up to $j_{\max }=L_{\max }+1$ and subtract the corresponding transitions without FSI and add finally the complete $t$-matrix obtained with a plane wave as final state, which we call the plane wave BorN approximation (PWBA). This is described in detail in [23]. For all kinematics considered in this work $L_{\max }=4$ was found to be sufficient, i.e. the final result did not change if we increased $L_{\max }$. As already mentioned, the higher partial waves are needed only in the vicinity of the quasi-free ridge because of the then slow convergence of the partial wave expansion. The formal expression for the $t$-matrix in PWBA is given in the Appendix F. We will now explain some details of the separate ingredients.

\section{A. $N N$-potential models}

As mentioned above, the two-body wave functions, needed for the calculation of the observables, are based on realistic $N N$-potentials. In past work $[1,2,4]$ we have employed a number of realistic potentials, such as NiJMEGEN [28], PARIs [29], Bonn ( $r$ - and $p$-space versions) [27], and ARGOnne $\mathrm{V}_{14}$ [30] potentials. In general, we found that the dependence of the observables on the choice of a realistic potential is rather moderate, in particular at low and medium energy and momentum transfers. For this reason we have chosen only one semi-modern potential, the BonN $p$-space 
model, for which a consistent meson exchange current has been constructed recently including all leading order relativistic contributions [26] to the current operators, boost and internal dynamics as explained below in Sect. IV D. This potential, as most of the realistic $N N$-potentials, is defined in purely nucleonic space without explicit $\Delta$-d.o.f. Since, however, we will also present results, where such $\Delta$-d.o.f. are treated explicitly in a coupled channel approach, we use in addition an interaction potential which has been constructed recently for this purpose [26] and which is based on the BonN $p$-space potential. Furthermore, we consider as a prototype of recent high precision potentials the ARGOnNe $\mathrm{V}_{18}$ potential [31] in the $r$-space code.

\section{B. One-body currents}

The one-nucleon current is derived from the nonrelativistic reduction of the DIRAC current, retaining the leading order relativistic contributions. The internal nucleon structure is taken care of by including the free on-shell nucleon e.m. form factors. In the $p$-space code we use the DIRAC and PAULI form, $F_{1}\left(Q^{2}\right)$ and $F_{2}\left(Q^{2}\right)$, respectively, where $Q^{2}=-q_{\mu}^{2}$. Explicit expressions are listed in the Appendix of [26]. The SACHs form with $G_{E}\left(Q^{2}\right)$ for the electric and $G_{M}\left(Q^{2}\right)$ for the magnetic form factor is used in the $r$-space code. It is obtained from the DiRAC-PAULI form by the transformation

$$
\begin{aligned}
& F_{1}\left(Q^{2}\right)=\tau\left(Q^{2}\right)\left(G_{E}\left(Q^{2}\right)+\frac{Q^{2}}{4 M^{2}} G_{M}\left(Q^{2}\right)\right), \\
& F_{2}\left(Q^{2}\right)=\tau\left(Q^{2}\right)\left(G_{E}\left(Q^{2}\right)-G_{M}\left(Q^{2}\right)\right),
\end{aligned}
$$

with $\tau\left(Q^{2}\right)=\left(1+Q^{2} / 4 M^{2}\right)^{-1}$ and neglecting terms of higher order beyond the leading relativistic order. Since the difference between these two forms of the one-body current is of higher relativistic order, the different treatments in the two codes does not matter as long as the leading order relativistic contributions are included and as long as the kinematics stays within the limits of validity of the truncated expansion keeping only the terms of leading relativistic order [32].

A variety of form factor parametrizations is available [33-37]. The largest uncertainty exists for $G_{E n}$. Indeed, one of the early motivations for investigating deuteron electrodisintegration with polarization degrees of freedom was that it could provide a nearly model independent method of determining the neutron electric form factor $G_{E n}$ [38-40]. Since in these studies the sensitivity of various observables with respect to $G_{E n}$ has been investigated extensively, we have employed here only one form factor model, namely the dipole model including a nonvanishing electric form factor of the neutron in the GALSTER parametrization [33] (with $\mathrm{p}=5.6$ ).

In principle, one should consider also off-shell effects in the one-body current in view of the fact, that the nucleons are not free but subject to the hadronic interaction. In such a situation, the form factors would acquire an additional dependence on the initial and final squared four-momenta of the nucleons and, moreover, additional currents with more off-shell form factors would appear. That such effects potentially may be non-negligible has been shown recently for deuteron photodisintegration [41]. In that study off-shell effects were evaluated using a simple, but not very realistic pion cloud model for the nucleon structure. At present, however, no realistic treatment of such off-shell form factors exists, and thus we neglect them here.

\section{Meson exchange currents}

An important property of realistic $N N$-interaction models is that they induce two-body meson exchange currents (MEC). Indeed, any isospin and/or momentum dependence of an $N N$-potential requires on a formal basis the existence of an interaction current in order to satisfy the continuity equation. The physics underlying such MECs is related to the coupling of the hadronic interaction diagrams to the e.m. field. In the case that the $N N$-potential is explicitly derived from a meson-exchange model, this connection is obvious and one obtains straightforwardly the associated nonrelativistic MEC, of which the $\pi$-MEC is the most important one, as well as the leading order relativistic two-body charge and current contributions.

However, for potential models, which in their medium range part use a phenomenological parametrization, the construction of a proper exchange current for the isospin dependent potential part is not unique, because the connection to the underlying physical process is obscured. For such cases, a recipe has been developed in the past independently by Riska [42] and by Buchmann et al. [43], which is inspired by the genuine meson exchange models. This recipe is based on the observation that the spin-isospin dependent central and tensor parts of a given $N N$-potential can be split into a pion- and rho-exchange-like potential for which the corresponding meson exchange currents are known. While the approach of RISKA is based on the momentum space representation of the potential and thus can be applied 
to any phenomenological potential, the method of BUCHMANN et al. was conceived for an $r$-space representation of the potential as a superposition of appropriate YUKAWA functions, and thus its application appeared to be limited to such type of potentials. But recently, this approach was extended to potentials with a more general radial behaviour by applying a LAPLACE transform [44].

This phenomenological method works for the nonrelativistic MEC reasonably well, but one should be aware, that its construction contains some inherent arbitrariness, because the $\pi$ - and $\rho$-MEC contain purely transverse pieces, which are not constrained by current conservation and thus can be modified arbitrarily without destroying the consistency. An example for such a modification is given in [43]. Thus, extending this recipe to the construction of relativistic MEC contributions in a corresponding manner has to be considered as purely heuristic. Fortunately, an important part of MEC can be incorporated model independently by the use of SIEGERT operators for the transverse electric multipoles $[45,46]$. In fact, in this way the major MEC contribution to electric transitions is consistently incorporated implicitly, at least for low and medium energy and momentum transfers. In fact, the results in our work $[1,2,4,5]$ labeled as the normal part (N) contain MEC contributions via the electric SIEGERT operators implicitly, but otherwise no explicit MEC in either the electric or magnetic transitions. However, as already mentioned, in the $p$-space code, based on the work of RiTz et al. [26], no SiEGERT operators are used. In this case the results labeled "N" do not contain any MEC implicitly. With respect to the isobar configurations, which will be discussed in the next section, we also include those isobar-MEC which are induced by the transition potentials.

A last remark concerns the question of e.m. form factors for the MEC. Here we take the heuristic approach multiplying the isoscalar and isovector pieces by the appropriate isoscalar and isovector nucleon form factors. In this way, current conservation holds also in the presence of such form factors.

\section{Isobar contributions}

Isobar d.o.f., describing phenomenologically internal nucleon d.o.f., can be incorporated either in the form of effective nonlocal two-body operators, describing intermediate excitations of one or two isobars, or by allowing explicit isobar configurations (IC) in the nuclear wave functions, where one or several nucleons are replaced by an isobar, and with appropriate strong and e.m. operators [47]. In the present work the latter approach is used by admitting isobars as explicit constituents in the two-body system. This allows one also to handle in a natural manner the real excitation of a $\Delta(1232)$-resonance for high enough energy transfers above pion production threshold. It furthermore avoids the often applied static approximation of the effective MEC induced by the intermediate excitation of an isobar, which has very limited value only. In the present work, we have included the $N \Delta, N N(1440)$, and $\Delta \Delta$ configurations in the $r$-space code while in the $p$-space code only the $N \Delta$ configuration is included as the most important one for energy transfers up to about $400 \mathrm{MeV}$.

The corresponding wave function components, called isobar configurations, are obtained either in a perturbative treatment or in a coupled channel approach. In the perturbative approach, an isobar configuration, consisting, for example of a $N \Delta$ configuration, is generated by just one $N N$-collision via a transition potential $N N \rightarrow N \Delta$ for which a simple one-boson exchange model is used [47]. On the other hand, in a coupled channel approach one has to renormalize the original $N N$-interaction as mentioned above, because being fitted in pure $N N$-space to experimental scattering data, it contains implicitly already the effect of such intermediate configurations, e.g. $N \Delta$. This means that in principle one would need to redo the fit of the potential parameters if such isobar configurations are included explicitly. In order to avoid such involved work, a reliable box-subtraction method, first proposed by GREEN and SAINIO [48], is available and was applied in [25, 26]. In most cases, however, we use the simpler perturbative calculation [47]. Only in case of the $N \Delta$-configuration do we also consider a coupled channel calculation for the BoNN $p$-space potential [26] as mentioned above, because in momentum space the $\Delta$ propagation can be treated in a more exact manner compared to an $r$-space calculation [25]. Finally, one- and two-body current operators involving isobars have to be considered. In view of the fact, that these currents are less well known, we neglect relativistic terms and restrict ourselves to their nonrelativistic expressions which are given in [47] for the one-body terms and in [49] for the MEC contributions, again with appropriate e.m. form factors.

\section{E. Relativistic contributions}

Relativistic contributions arise from three sources. These are (i) the internal relativistic dynamics in the rest frame of the nucleus, (ii) the boost of the intrinsic wave function from the nuclear rest frame, here the lab frame, to a moving one because of the nonvanishing momentum transfer, and finally (iii) relativistic contributions to the interaction operators, here the current operators. In the present approach we resort to a $p / M$-expansion, retaining only the leading order relativistic terms beyond the nonrelativistic limit. The boost of a wave function is described 
by a unitary operator for which the $p / M$-expansion yields in leading order two separate contributions, a purely kinematic part, which can be interpreted as the effect of LORENTZ contraction and THOMAS-WigNER spin rotation, and a potential dependent part, which only is present for pseudoscalar meson exchange [50]. Relativistic contributions to the one-body current have been discussed already in Sect. IV B. With respect to MEC, consistent treatments are available in [51-54] which are based on a meson-theoretical one-boson-exchange potential as nuclear interaction. The construction is more questionable for semi-phenomenological potentials like the ARGONNE $V_{18}$, as pointed out in Sect. IV C. Even a consistent nonrelativistic MEC is ambiguous for such potentials [42]. Although one can proceed also for the relativistic MEC in analogy to purely one-boson-exchange models, one should be aware of the inherent ambiguities of such an approach.

A consistent treatment of all three types of contributions is given in the work of GölLER and ARENHÖVEL [52] for a pure one-pion-exchange model and by TAMURA et al. [53] for a more general potential type. The work of [52] has been generalized in [26] to a consistent leading order relativistic treatment for the $p$-space BonN potentials, and our $p$-space code is based on this work. In view of the problems associated with the construction of a consistent MEC for a semi-phenomenological potential, we include in the $r$-space code as relativistic current contribution only the one-body part, containing the most important spin-orbit current, and the kinematic boost as described in [55].

\section{DISCUSSION OF RESULTS}

In the following discussion we will use as shorthand "FSI" for final state interaction, "PWBA" for nonrelativistic one-body current with plane wave final state, "RPWBA" for relativistic one-body current with plane wave final state, "N" for the nonrelativistic normal theory, i.e. without explicit meson exchange currents and isobar configurations. This means in the case that SIEGERT operators are used, that a part of MEC is implicitly included in the electric multipoles for " $\mathrm{N}$ " as is the case for the $r$-space code, whereas for the $q$-space code no MEC contributions in "N" appear. Furthermore, "MEC" and "IC" stand for the contributions of explicit meson exchange currents and isobar configurations, respectively, "RC" for the inclusion of relativistic contributions and "T=N+MEC+IC+RC" for the complete calculation.

\section{A. Inclusive Observables}

The inclusive reaction $d\left(e, e^{\prime}\right) n p$ for unpolarized beam and target is governed by a longitudinal form factor $F_{L}$ and a transverse one $F_{T}$, whereas eight additional form factors appear if one allows for beam and target polarization. These form factors depend on two variables, for which we choose the final state c.m. excitation energy $E_{n p}$ (see Eq. (1)) and the squared c.m. three-momentum transfer $\vec{q}^{2}$ (from now on we use the notation $\vec{q}=\vec{q}^{\text {c.m. }}$ ), i.e. $F_{L / T}=F_{L / T}\left(E_{n p}, \vec{q}^{2}\right)$. All results presented in Figs. 3 through 11 are obtained using the ARGONNE $V_{18}$ potential. In order to give an overview, the upper panels of Fig. 3 show $F_{L}$ and $F_{T}$, calculated with the complete theory, in combined surface and contour plots over the $E_{n p^{-}} q^{2}$-plane for $E_{n p}=0-300 \mathrm{MeV}$ and $q^{2}=0-25 \mathrm{fm}^{-2}$. For a more detailed view of the form factors in the near threshold region the lower panels display them in a smaller part of the $E_{n p^{-}} q^{2}$-plane, namely for $E_{n p}=0-20 \mathrm{MeV}$ and $q^{2}=0-2 \mathrm{fm}^{-2}$.

One readily notes that the quasi-free ridge along $E_{n p} / \mathrm{MeV} \approx 10 q^{2} / \mathrm{fm}^{-2}$ is the dominant feature of these two form factors, where the quasi-free kinematic is defined by the requirement that the virtual exchanged photon is absorbed by only one nucleon, which is emitted in the forward direction, with energy and momentum transfer such that the spectator nucleon remains at rest in the lab system. This yields the condition

$$
E_{n p}^{\mathrm{lab}}=M+\sqrt{M^{2}+\left(q^{\mathrm{lab}}\right)^{2}}
$$

which gives for the invariant mass according to (4a) using (9b)

$$
W_{n p}=2 M \sqrt{1+\frac{q^{2}}{M_{d}^{2}}} \approx M\left(2+\frac{q^{2}}{M_{d}^{2}}\right)
$$

or for the final state c.m. excitation energy

$$
E_{n p}=2 M\left(\sqrt{1+\frac{q^{2}}{M_{d}^{2}}}-1\right) \approx \frac{M}{M_{d}^{2}} q^{2} .
$$



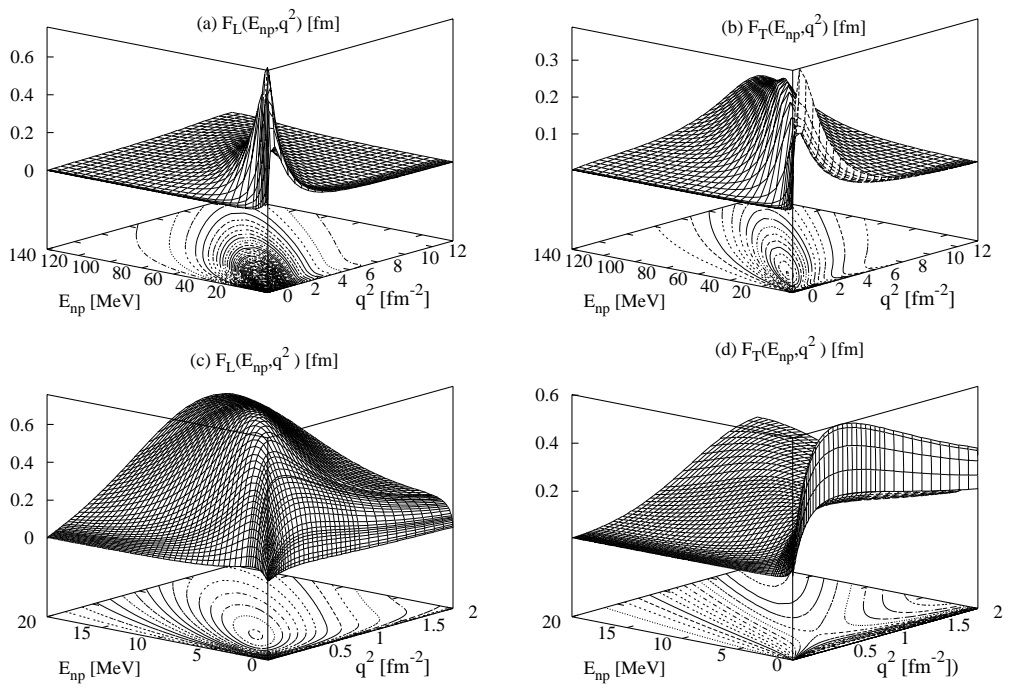

FIG. 3: Surface and contour plots of longitudinal and transverse form factors as function of $E_{n p}$ and $q^{2}$ for $E_{n p}=0-300 \mathrm{MeV}$ and $q^{2}=0-25 \mathrm{fm}^{-2}$ using the Argonne $V_{18}$ potential.
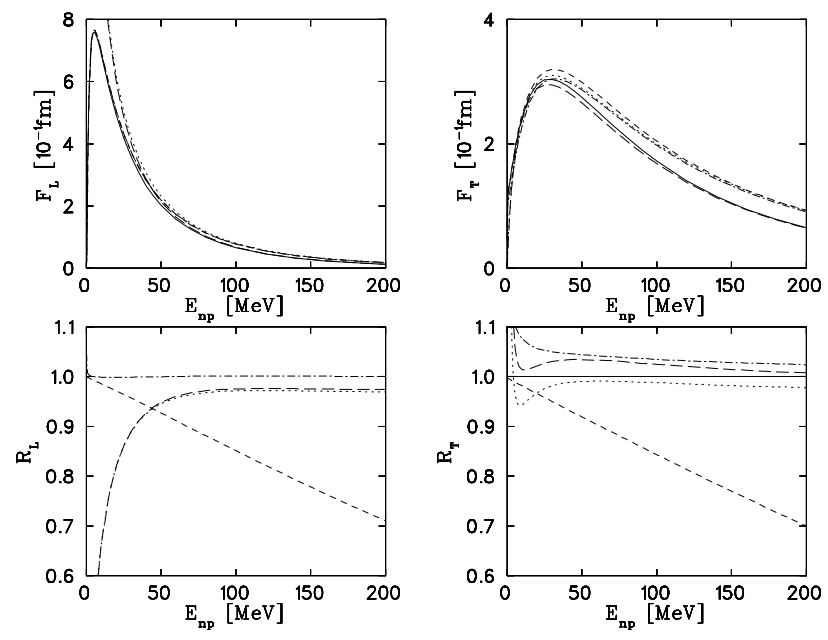

FIG. 4: Upper panels: Longitudinal and transverse form factors along the quasi-free ridge as function of $E_{n p}$ calculated using the ARgonne $\mathrm{V}_{18}$ potential. Notation: dotted: PWBA; long dashed: RPWBA; dash-dot: normal (N), i.e. nonrelativistic approach with FSI included; short dashed: nonrelativistic MEC and IC included (N+MEC+IC); solid: complete calculation $(\mathrm{T}=\mathrm{N}+\mathrm{MEC}+\mathrm{IC}+\mathrm{RC})$. Lower panels: Form factor ratios $R_{L / T}=F_{L / T}(\mathrm{~N}) / F_{L / T}(\mathrm{PWBA})($ dotted), $R_{L / T}=F_{L / T}(\mathrm{~T}) / F_{L / T}(\mathrm{RPWBA})$ (long dashed) $R_{L / T}=F_{L / T}(\mathrm{~N}+\mathrm{MEC}+\mathrm{IC}) / F_{L / T}(\mathrm{~N})($ dash-dot$)$, and $R_{L / T}=$ $F_{L / T}(\mathrm{~T}) / F_{L / T}(\mathrm{~N}+\mathrm{MEC}+\mathrm{IC})$ (short dashed).

The latter relation gives the already mentioned rule of thumb $E_{n p} / \mathrm{MeV} \approx 10 q^{2} / \mathrm{fm}^{-2}$, a straight line in the $E_{n p^{-}} q^{2}-$ plane as is obvious in Fig. 3.

The behaviour of the form factors along the quasi-free ridge is displayed in the upper two panels of Fig. 4 while the lower two panels show form factor ratios with respect to the various ingredients and interaction effects. The longitudinal form factor rises steeply, reaches its maximum at quite low $E_{n p}$ around $5 \mathrm{MeV}$, and falls off very rapidly with increasing $E_{n p}$. On the other hand, $F_{T}$ rises considerably slower and reaches its maximum only around $E_{n p}=30 \mathrm{MeV}$. Also the fall off is much slower compared to $F_{L}$. One furthermore notes that for $F_{L}$ the effect of MEC and IC is unimportant while for $F_{T}$ these are still sizeable above $E_{n p}=20 \mathrm{MeV}$ and of the order of several percent decreasing slowly with growing $E_{n p}$ as shown by the dash-dotted curves in the lower panels. Also the influence of FSI becomes quite unimportant above $E_{n p} \approx 30 \mathrm{MeV}$ as seen in the upper panels by comparing the dotted curves (nonrelativistic PWBA) with the short dashed curves $(\mathrm{N}+\mathrm{MEC}+\mathrm{IC})$. Quantitatively one finds from the ratios in the 

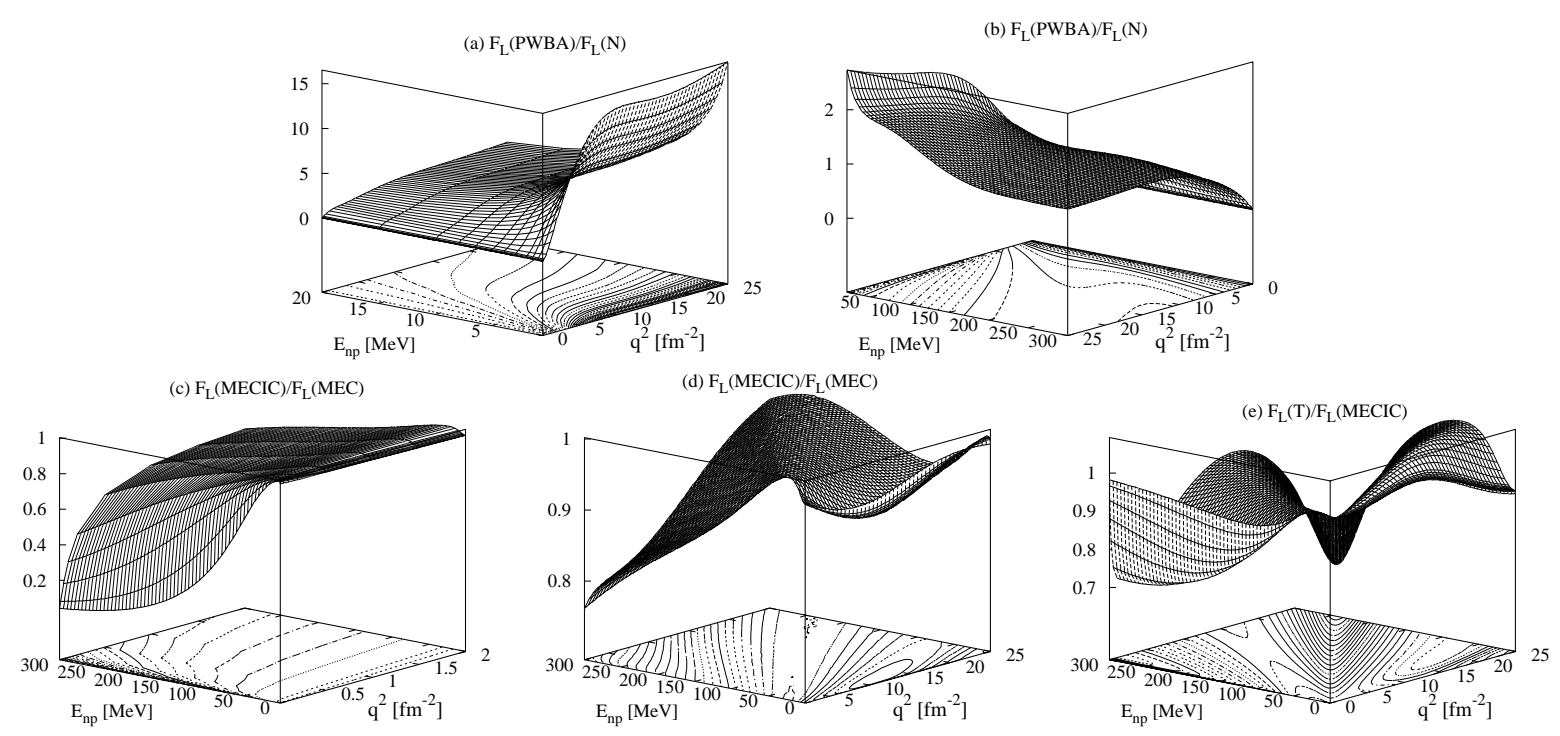

FIG. 5: Surface and contour plots over the $E_{n p^{-}} q^{2}$-plane of ratios of longitudinal form factors for different contributions: (a) influence of FSI, $F_{L}(\mathrm{PWBA}) / F_{L}(\mathrm{~N})$, for low $E_{n p}=0-20 \mathrm{MeV}$ and in (b) for $E_{n p}=20-300 \mathrm{MeV}$; (c) influence of IC, $F_{L}(\mathrm{MECIC}) / F_{L}(\mathrm{MEC})$, for low $q^{2}=0-2 \mathrm{fm}^{-2}$ and in $(\mathrm{d})$ for $q^{2}=2-25 \mathrm{fm}^{-2}$; (e) influence of $\mathrm{RC}, F_{L}(\mathrm{~T}) / F_{L}(\mathrm{MECIC})$.

lower panels (dotted curves) that above $E_{n p} \approx 100 \mathrm{MeV}$ an almost constant difference of a few percent remains. The analogous ratios with relativistic contributions included, i.e. $F_{L / T}(\mathrm{~T}) / F_{L / T}(\mathrm{RPWBA})$ show a similar behaviour (long dashed curves). The reason for the large overestimation of $F_{L}$ in PWBA at low $E_{n p}$ has its origin in the fact that the final state plane wave is not orthogonal to the deuteron bound state so that the charge monopole transition is not suppressed near threshold. On the other hand, $F_{T}$ is strongly underestimated in PWBA because of the absence of the resonance in the ${ }^{1} S_{0}$ state. The only notable effect arises from relativistic contributions leading for both form factors to a sizeable reduction which increases almost linearly with $E_{n p}$. However, these RC are quite well accounted for in the relativistic RPWBA as is demonstrated by the little difference between the complete calculation and the relativistic RPWBA their ratios approaching one with increasing $E_{n p}$ (long dashed curves).

We now will turn to the near threshold behaviour shown in the lower panels of Fig. 3. One readily notes that for $E_{n p} \rightarrow 0$ along $q^{2}=$ const. $F_{L}$ runs first through a broad maximum and then decreases rapidly to zero while $F_{T}$ rises dramatically resulting in a very sharp peak right above threshold. The peak height grows first with increasing $q^{2}$, reaches its maximum around $q^{2}=0.5 \mathrm{fm}^{-2}$ and then falls off. The rapid decrease of $F_{L}$ and the sharp peak of $F_{T}$ near $E_{n p} \approx 0$ is a consequence of the fact, that close to break-up threshold the ${ }^{1} S_{0}$-scattering state, the so-called anti-bound state, dominates the final state into which the Coulomb monopole transition is forbidden while one has a very strong magnetic dipole isovector transition which is further enhanced by MEC and IC contributions. Above $E_{n p} \approx 4 \mathrm{MeV}$ and $q^{2} \approx 0.4 \mathrm{fm}^{-2}$ both the surface plot as well as the contour lines exhibit clearly the onset of the quasi-free ridge in both form factors.

The relative influence of the various interaction effects are shown as ratios in Fig. 5 for the longitudinal form factor. Because of the strong influence of FSI on $F_{L}$ near threshold, we show for this form factor the ratio $F_{L}(\mathrm{PWBA}) / F_{L}(\mathrm{~N})$ separately for the region $E_{n p} \leq 20 \mathrm{MeV}$ in Fig. 5 (a), exhibiting the already noted very strong effect of FSI, resulting in a strong increase of this ratio for $q^{2} \rightarrow 0$ along $E_{n p}=$ const., whereas $F_{L}(\mathrm{PWBA}) / F_{L}(\mathrm{~N}) \rightarrow 0$ for $E_{n p} \rightarrow 0$ along $q^{2}=$ const. For the remaining part $\left(20 \leq E_{n p} / \mathrm{MeV} \leq 300\right)$ this ratio is shown in Fig. 5 (b). The influence of FSI is minimal on top of the quasi-free ridge while it leads to an increase above this ridge, i.e. for $E_{n p} / \mathrm{MeV}>10 q^{2} / \mathrm{fm}^{-2}$, and to a decrease below. MEC have almost no effect on $F_{L}$ and thus are not shown here, because the dominant nonrelativistic $\pi$-MEC does not contribute to the charge density. The effect of isobar configurations on $F_{L}$ is shown in Fig. 5 (c) for $q^{2} \leq 2 \mathrm{fm}^{-2}$ and in (d) for $q^{2} \geq 2 \mathrm{fm}^{-2}$. Above the quasi-free ridge $F_{L}$ is reduced by IC. In particular for low $q^{2}$ close to zero and $E_{n p}$ approaching the $\Delta$-excitation region, $F_{L}$ decreases drastically by IC to about 10 percent. Going below the quasi-free ridge, one again notes a reduction but of smaller size which diminishes when approaching small $E_{n p}$. Furthermore, RC, shown in Fig. 5 (e), exhibit an interesting behaviour: along the quasi-free ridge one finds a distinctive valley describing the increasing reduction by $\mathrm{RC}$ with increasing $E_{n p}$ or $q^{2}$ as was already apparent in Fig. 4 (upper right panel). Away from the quasi-free ridge the influence of RC diminishes first on both sides to almost zero and increases then again when approaching the regions of higher $E_{n p}$ and lower $q^{2}$ or vice versa. 

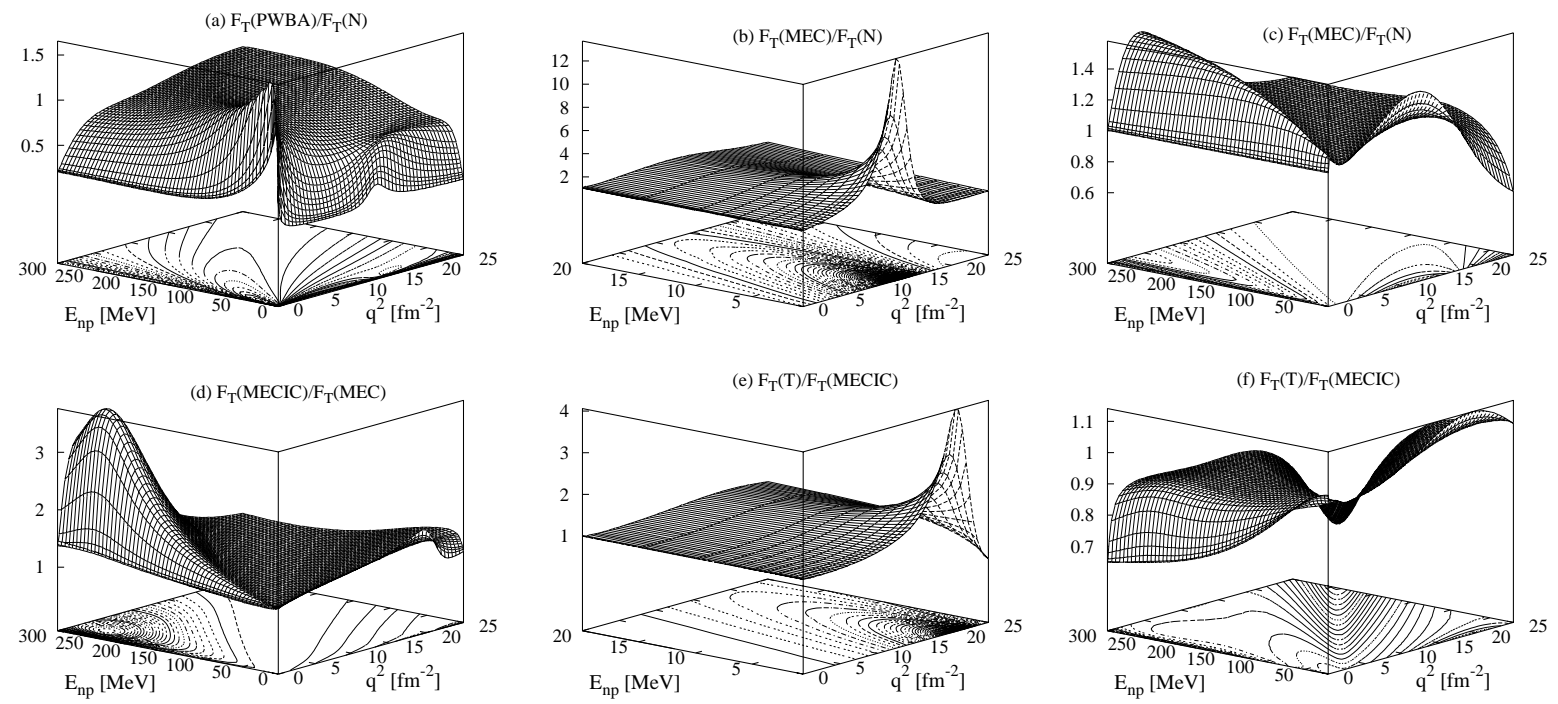

FIG. 6: Surface and contour plots over the $E_{n p}-q^{2}$-plane of ratios of transverse form factors for different contributions: (a) influence of FSI, $F_{T}(\mathrm{PWBA}) / F_{T}(\mathrm{~N})$; (b) influence of MEC, $F_{T}(\mathrm{MEC}) / F_{T}(\mathrm{~N})$ for low $E_{n p}=0-20 \mathrm{MeV}$ and in $(\mathrm{c})$ for $E_{n p}=$ $20-300 \mathrm{MeV}$; (d) influence of IC, $F_{T}$ (MECIC) $/ F_{T}$ (MEC); (e) influence of RC, $F_{T}(\mathrm{~T}) / F_{T}(\mathrm{MECIC})$ for low $E_{n p}=0-20 \mathrm{MeV}$ and in (f) for $E_{n p}=20-300 \mathrm{MeV}$.

The corresponding ratios for $F_{T}$ are displayed in Fig. 6. For this form factor the effect of FSI is much more pronounced off the quasi-free ridge than in $F_{L}$ as is shown by the ratio $F_{T}(\mathrm{PWBA}) / F_{T}(\mathrm{~N})$ in part (a). One finds a very strong decrease on both sides, i.e. at low $E_{n p}$ with increasing $q^{2}$ as well as at low $q^{2}$ but increasing $E_{n p}$, which means a strong enhancement by FSI. For the display of MEC effects we show in part (b) the region of low $E_{n p}$ and in part (c) the remaining region. As already mentioned, along the quasi-free ridge one finds little influence. But going away from this ridge, MEC lead to a sizeable increase as shown in part (c), especially strong close to threshold (see part (b)) up to about $q^{2}=15 \mathrm{fm}^{-2}$. For higher momentum transfers MEC result in a reduction. This behaviour is well known and in agreement with experimental data [56, 57]. Isobar effects displayed in Fig. 6 (d) become quite pronounced only in the region of $\Delta$ excitation near $E_{n p}=260 \mathrm{MeV}$ and for not too high momentum transfers. Finally, relativistic contributions lead for $E_{n p}$ near threshold and $q^{2}$ around $15-20 \mathrm{fm}^{-2}$ to quite a significant increase as shown in Fig. 6 (e). For the remaining region in Fig. 6 (f) one notes again a significant reduction along the quasi-free ridge as in $F_{L}$ in the form of a pronounced valley.

A survey for the additional form factors for polarized beam and target, calculated with the complete theory, is shown in Fig. 7 for $F_{L}^{20}, F_{T}^{20}, F_{T}^{\prime 10}$, and $F_{T T}^{2-2}$ and in Fig. 8 for the $L T$-interference form factors as surface and contour plots over a smaller portion of the $E_{n p^{-}} q^{2}$-plane, i.e. $E_{n p}=0-160 \mathrm{MeV}$ and $q^{2}=0-16 \mathrm{fm}^{-2}$. The largest polarization form factors are $F_{T}^{20}, F_{T T}^{2-2}, F_{T}^{\prime 10}$, and $F_{L T}^{\prime 1-1}$ which are of the same order of magnitude as the unpolarized form factors. An order of magnitude smaller are $F_{L}^{20}$ and $F_{L T}^{2-1}$. The remaining two, $F_{L T}^{1-1}$ and $F_{L T}^{\prime 2-1}$, are three orders of magnitude smaller although they increase slightly in size along the quasi-free ridge.

The influence of FSI, MEC, IC and RC cannot be displayed as ratios because these form factors have zeros. For this reason we show only for the larger form factors the most important influences by plotting differences for FSI (N-PWBA) in Fig. 9, for combined contribution from MEC and IC (MECIC-N) in Fig. 10, and for RC (T-MECIC) in Fig. 11. Final state interaction is very important for all polarization form factors shown in Fig. 9 in the region of low $E_{n p}=0-40 \mathrm{MeV}$ and low $q^{2}=0-4 \mathrm{fm}^{-2}$. MEC and IC effects displayed in Fig. 10 are substantial in the transverse form factors $F_{T}^{20}, F_{T T}^{2-2}$, and $F_{T}^{\prime 10}$, mostly below the quasi-free ridge, whereas they are of minor importance in the interference form factors $F_{L T}^{2-1}$ and $F_{L T}^{\prime 1-1}$, the largest effect being near the quasi-free ridge. Relativistic contributions show up in Fig. 11 along the quasi-free ridge in the primed form factors $F_{T}^{\prime 10}$ and $F_{L T}^{\prime 1-1}$ as well as in $F_{L T}^{2-1}$.

Now we will turn to a comparison with experimental data. Most extensively discussed has been the inclusive electrodisintegration near threshold, averaged over $E_{n p}=0$ through 3-5 MeV, at higher momentum transfers (see [56, 57]) and we will not repeat the discussion here. Instead we will compare the theory to the experimental data for $F_{L}$ and $F_{T}$ as obtained by a Rosenbluth separation in the near threshold region for various momentum transfers by SIMON et al. [58]. The only comparison to theory reported in that work was for a nonrelativistic treatment employing the PARIS potential and $\pi$-MEC, and a satisfactory agreement was found. In the light of the considerable progress 

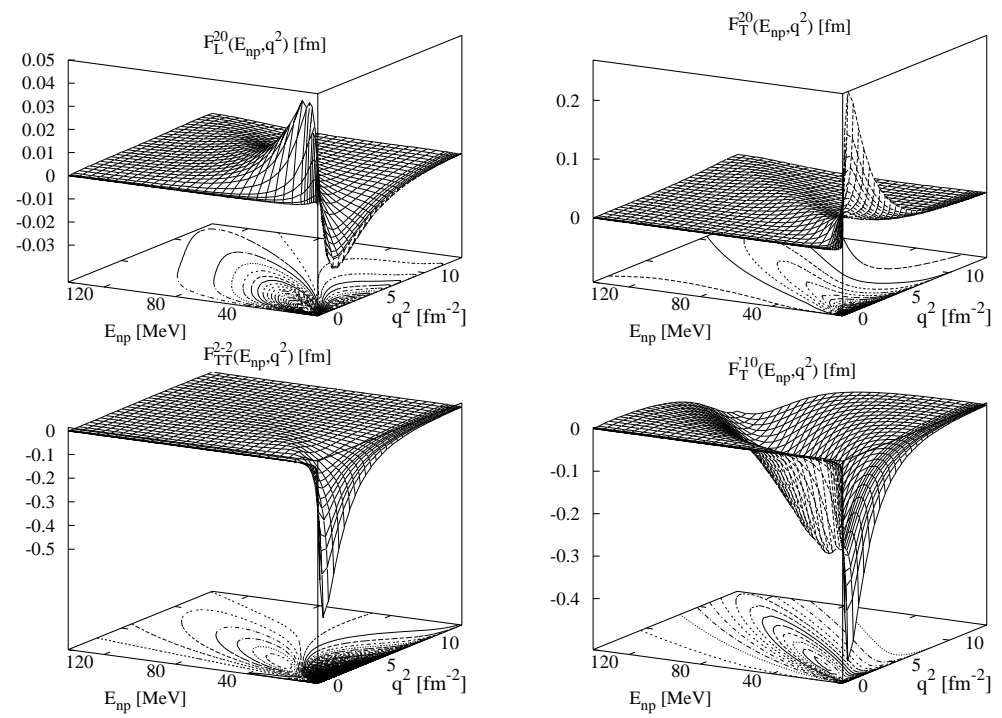

FIG. 7: Surface and contour plots of polarization form factors $F_{L}^{20}, F_{T}^{20}, F_{T}^{\prime 10}$, and $F_{T T}^{2-2}$ for polarized beam and target as function of $E_{n p}$ and $q^{2}$ for $E_{n p}=0-160 \mathrm{MeV}$ and $q^{2}=0-16 \mathrm{fm}^{-2}$.
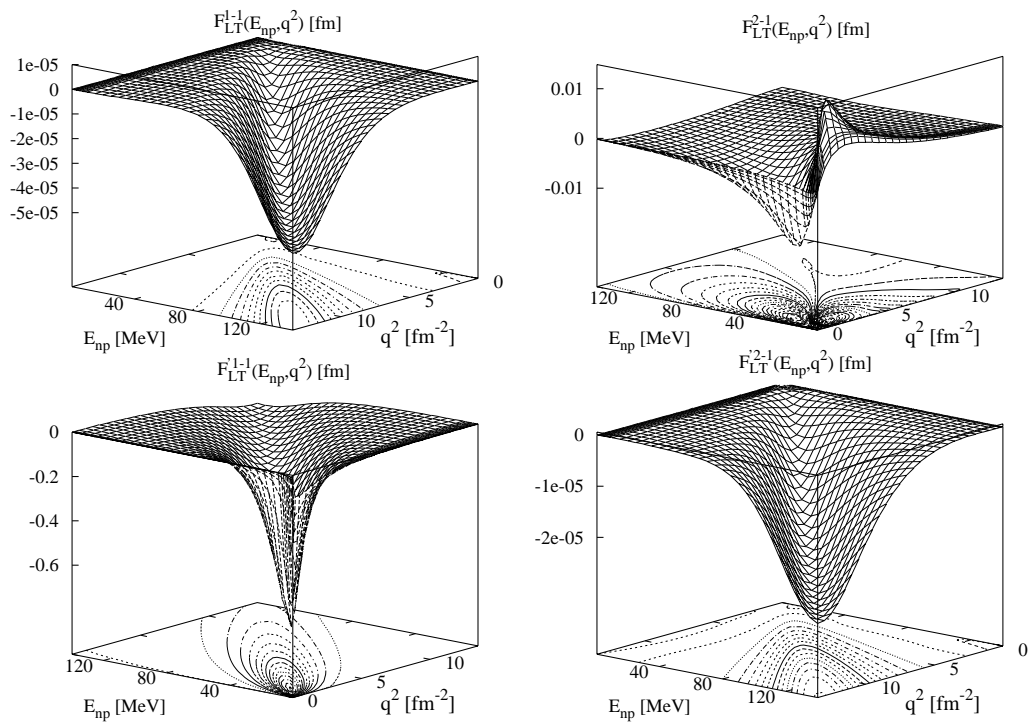

FIG. 8: Surface and contour plots of polarization $L T$-interference form factors for polarized beam and target as function of $E_{n p}$ and $q^{2}$ for $E_{n p}=0-160 \mathrm{MeV}$ and $q^{2}=0-16 \mathrm{fm}^{-2}$.

which theory has achieved since then, it appears timely to compare the modern approaches with those data. Thus we confront in Fig. 12 three data sets of [58] corresponding to the squared momentum transfers $q^{2} \approx 0.6,1.5$, and $3.8 \mathrm{fm}^{-2}$ for $E_{n p}=0-9 \mathrm{MeV}$ with various interaction effects, i.e. normal nonrelativistic approach (N), with inclusion of meson exchange currents and isobar configurations $(\mathrm{N}+\mathrm{MEC}+\mathrm{IC})$, and the complete theory including relativistic contributions $(\mathrm{T}=\mathrm{N}+\mathrm{MEC}+\mathrm{IC}+\mathrm{RC})$ for the BonN-Qb potential.

As expected, for $F_{L}$ the interaction effects from MEC and IC are very small though not completely negligible. Relativistic effects show some influence resulting in a small reduction above $E_{n p} \approx 5 \mathrm{MeV}$ which increases slightly in size with $E_{n p}$. The agreement of the full theory with the data for $F_{L}$ is quite satisfactory. One should keep in mind, that no open parameter has been fit in this comparison.

The transverse form factor $F_{T}$ shows right above threshold the already noted distinctive peak arising from the $M 1$-transition into the anti-bound ${ }^{1} S_{0}$-state. Here the influence of MEC and IC is quite significant, especially in the peak region, and it grows sizeably with increasing squared momentum transfer. On the other hand, RC lead only to 

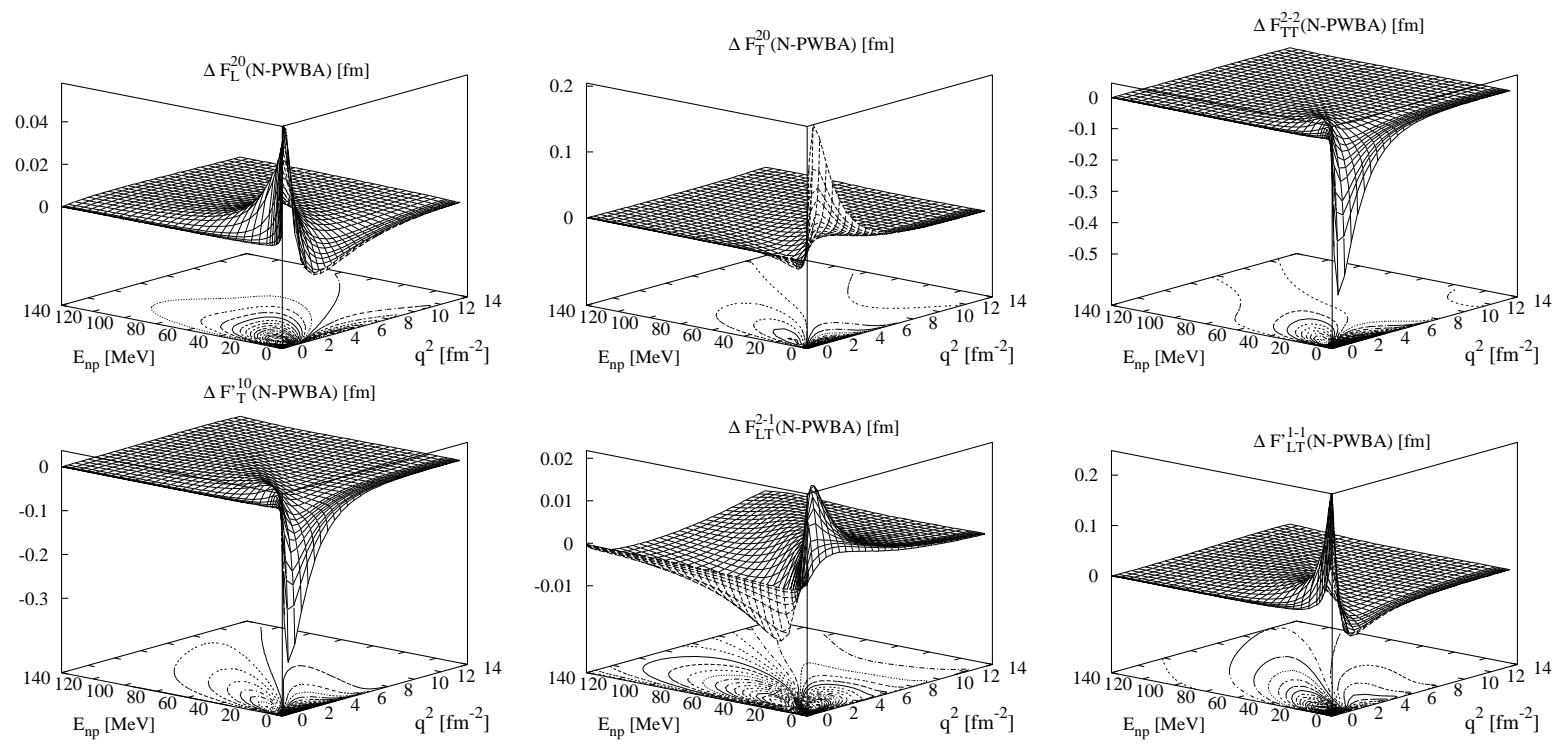

FIG. 9: Influence of FSI: Surface and contour plots of differences of polarization form factors calculated in normal nonrelativistic theory $(\mathrm{N})$ and in PWBA as function of $E_{n p}$ and $q^{2}$ for $E_{n p}=0-140 \mathrm{MeV}$ and $q^{2}=0-14 \mathrm{fm}^{-2}$.
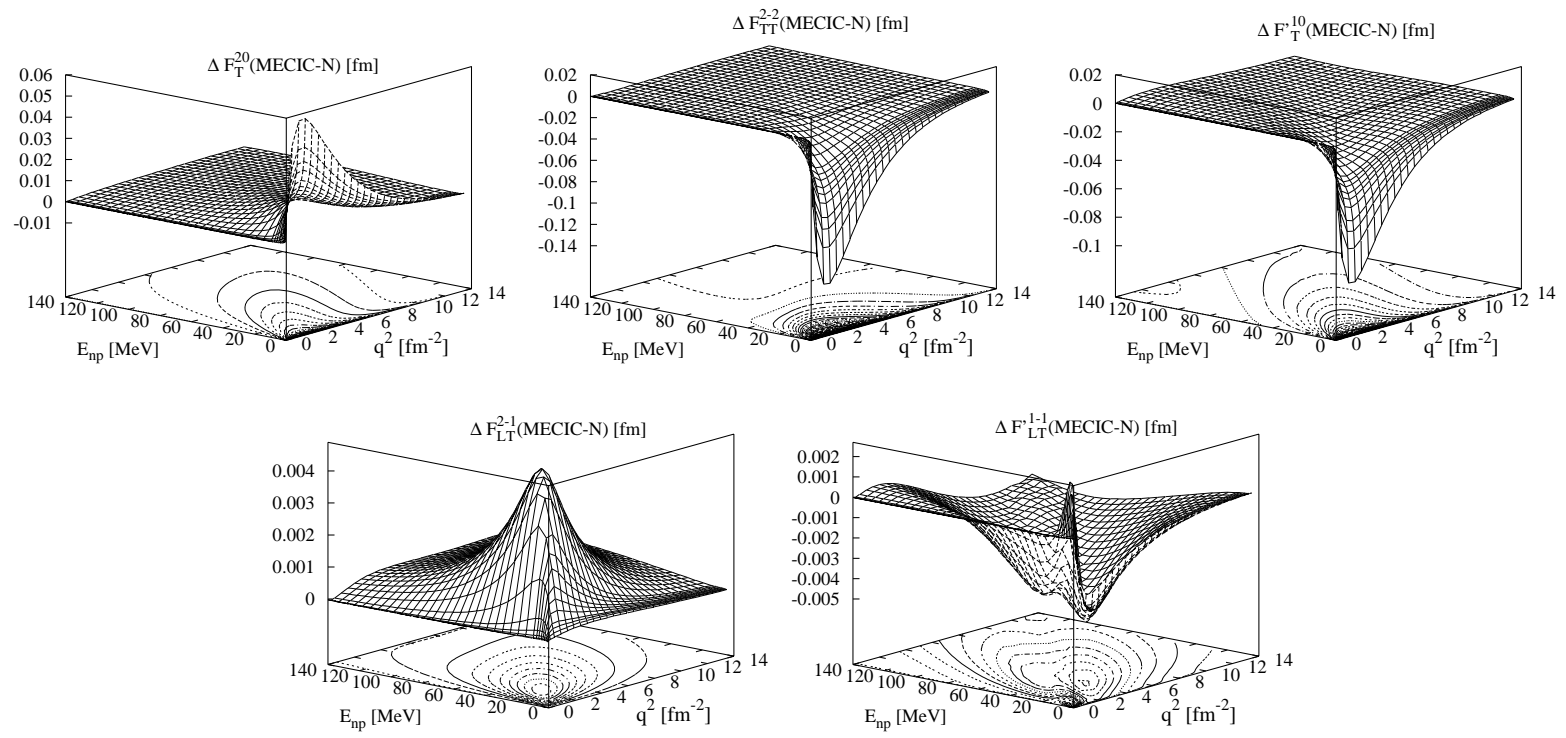

FIG. 10: Influence of MEC and IC: Surface and contour plots of differences of polarization form factors calculated with MEC and IC and in normal nonrelativistic theory as function of $E_{n p}$ and $q^{2}$ for $E_{n p}=0-140 \mathrm{MeV}$ and $q^{2}=0-14 \mathrm{fm}^{-2}$.

a slight reduction for $q^{2}=3.8 \mathrm{fm}^{-2}$ while for the other two cases they show no effect at all. The agreement with the data is in general quite good except for the peak where the theory lies above the data. It is very likely, however, that the experimental resolution was insufficient to resolve this very sharp peak. The potential model dependence is shown in Fig. 13 using the Bonn-Qb, the two ArgOnNe potentials $\mathrm{V}_{14}$ and $\mathrm{V}_{18}$, and the PARIS potential. The variation of the predicted form factors by these potentials is much smaller in size than the size of interaction effects. The only exception is the prediction of the peak height for the PARIS potential, which gives a lower value. The reason for this lies in the PARIS potential's prediction of too small a value for the $n p$-scattering length. Finally, in order to give a more detailed and quantitative comparison with experiment we show in Fig. 14 the ratios of the data and the various theoretical model predictions to the results obtained with the BonN-QB potential. It is obvious that the variation with the potential model is substantially smaller than the experimental errors. Certainly, much more precise data, in particular at the threshold peak, are needed in order to put the theory to a more critical test. 

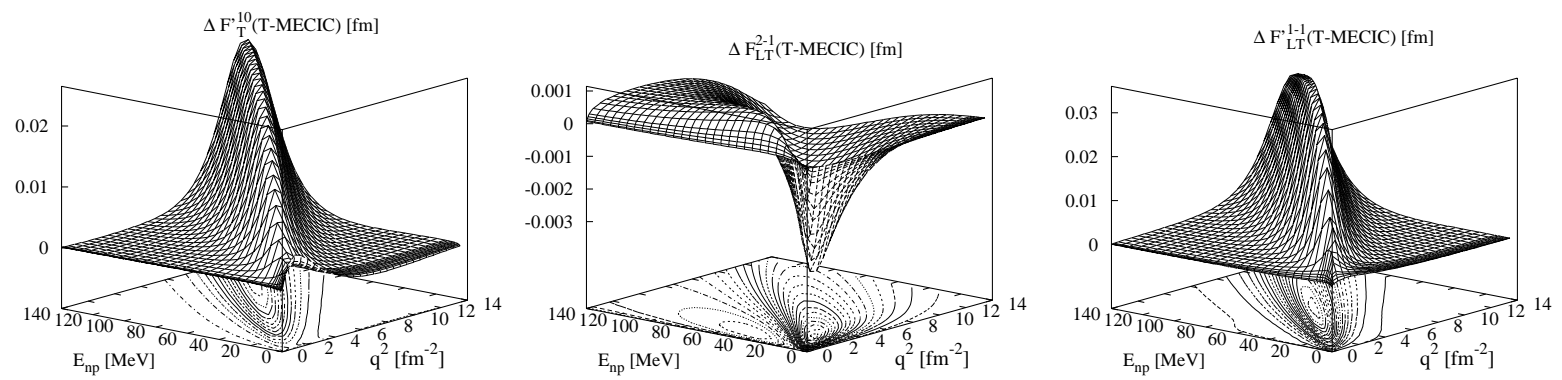

FIG. 11: Influence of RC: Surface and contour plots of differences of polarization form factors calculated with MEC and IC and for complete theory as function of $E_{n p}$ and $q^{2}$ for $E_{n p}=0-140 \mathrm{MeV}$ and $q^{2}=0-14 \mathrm{fm}^{-2}$.
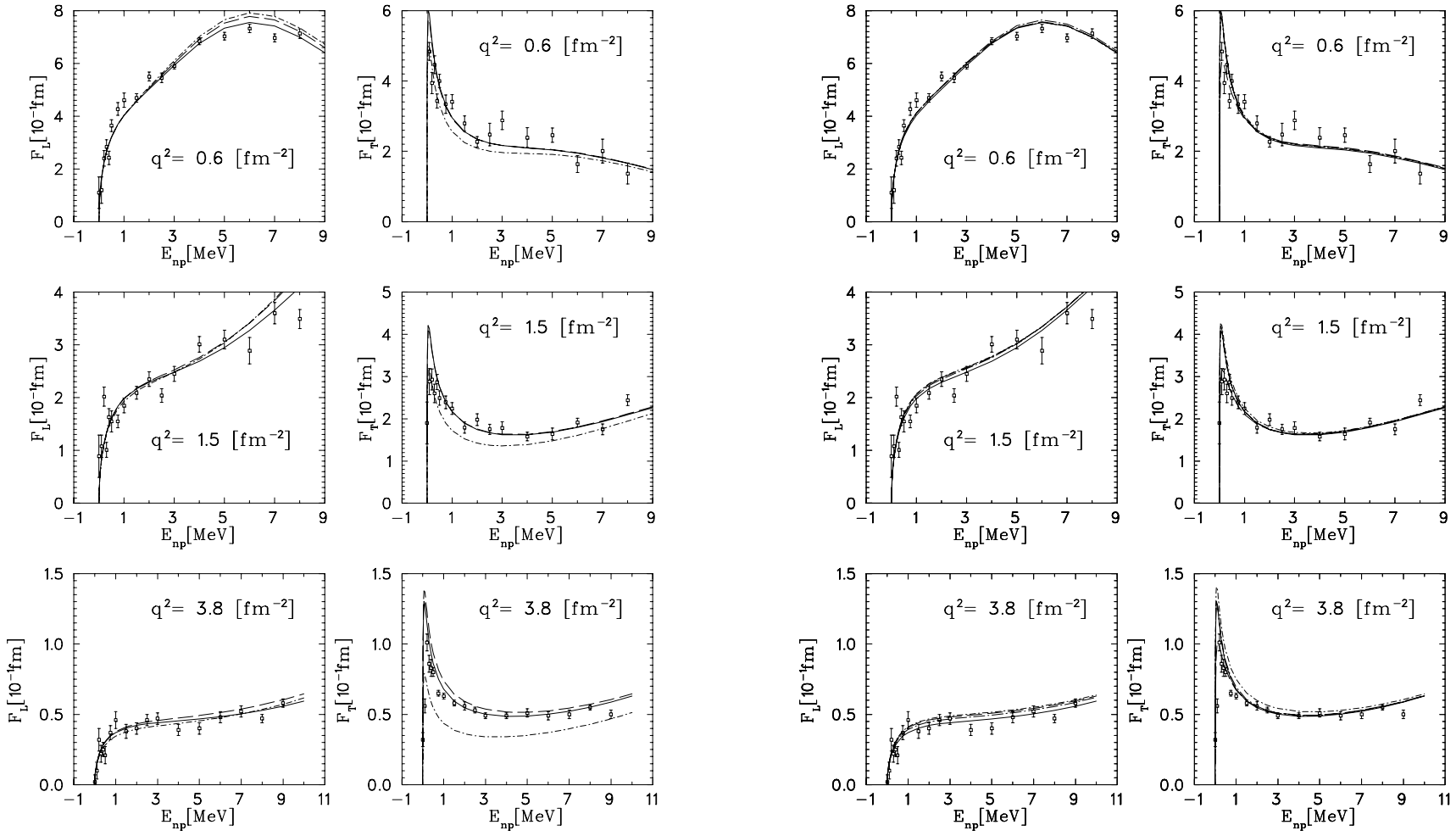

FIG. 12: Longitudinal and transverse form factors with various interaction effects. Notation: dash-dot: N; dashed: N+MEC+IC; solid: N+MEC+IC+RC. Experimental data from Simon et al. [58].

FIG. 13: Longitudinal and transverse form factors for various potential models. Notation: dashed: PARIS; long dashdot: Argonne $\mathrm{V}_{14}$; short dash-dot: Argonne $\mathrm{V}_{18}$; solid: BonN-Qb. Experimental data from from Simon et al. [58].

Another set of data for the inclusive reaction at higher excitation energy $E_{n p}$ and higher momentum transfers is provided by QuinN et al. [59]. We show first a comparison between theory and experiment for some inclusive cross sections corresponding to several different kinematics reported by QuinN et al. which exhibit nicely the quasi-free peak, namely at $E_{n p} \approx 20 \mathrm{MeV}$ in Fig. 15 and at $E_{n p} \approx 70 \mathrm{MeV}$ in Fig. 16. The upper panels refer to forward angles $\left(60^{\circ}\right)$ and the lower ones to backward angles $\left(134.5^{\circ}\right)$. In the left panels the predictions of the nonrelativistic normal theory obtained with the BonN-Qb and ARgOnNe $\mathrm{V}_{18}$ potentials is compared to the data and in the right panels the complete theory. The normal theory results in a slight potential dependence as is apparent in the quasi-free peak with the results for the BoNN-Qb potential slightly higher and more pronounced for the $60^{\circ}$-data. However, this potential model dependence is very much reduced for the complete theory. The most significant improvement of the full calculation is seen in the near threshold region at backward scattering angles (see lower panels). In general the agreement is quite good. The underestimation above $\omega=200 \mathrm{MeV}$ in the upper panels of Fig. 16 has its origin in the absence of pion production contributions in the theory which become significant in this region.

The fact, that the model dependence of the nonrelativistic normal theory is stronger for forward scattering angles, 

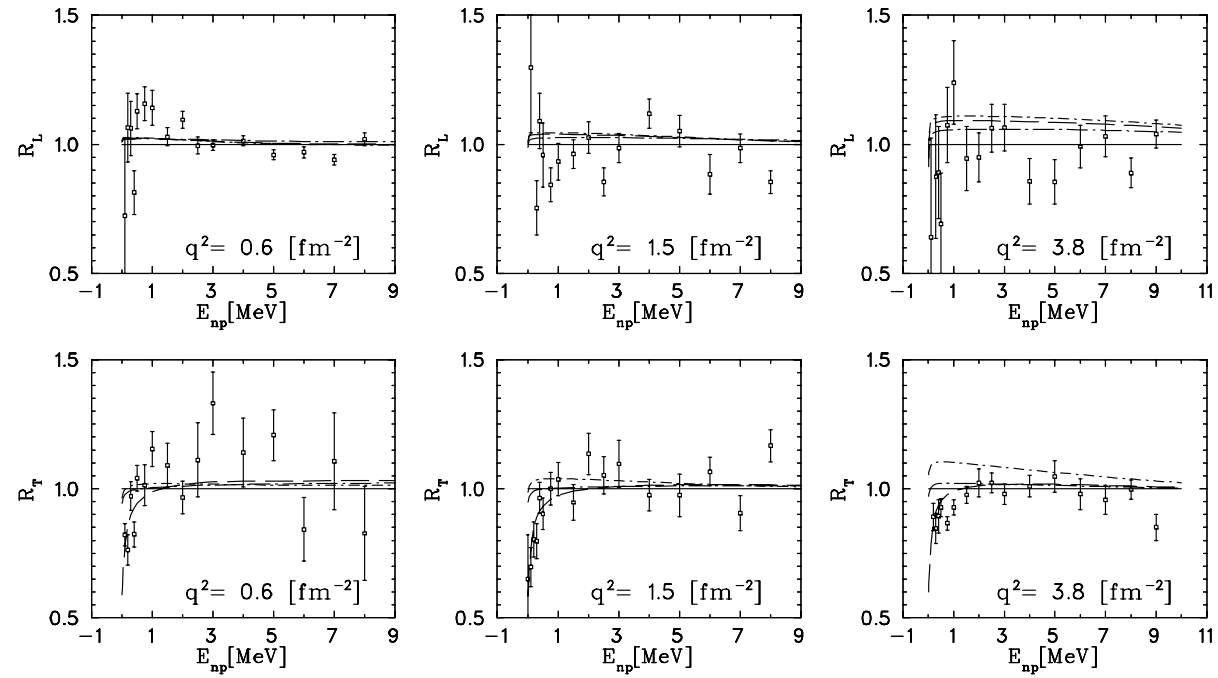

FIG. 14: Ratios of the longitudinal and transverse form factors for various potential models with respect to the BonN-Qb potential. Notation as in Fig. 13. Experimental data from from Simon et al. [58].
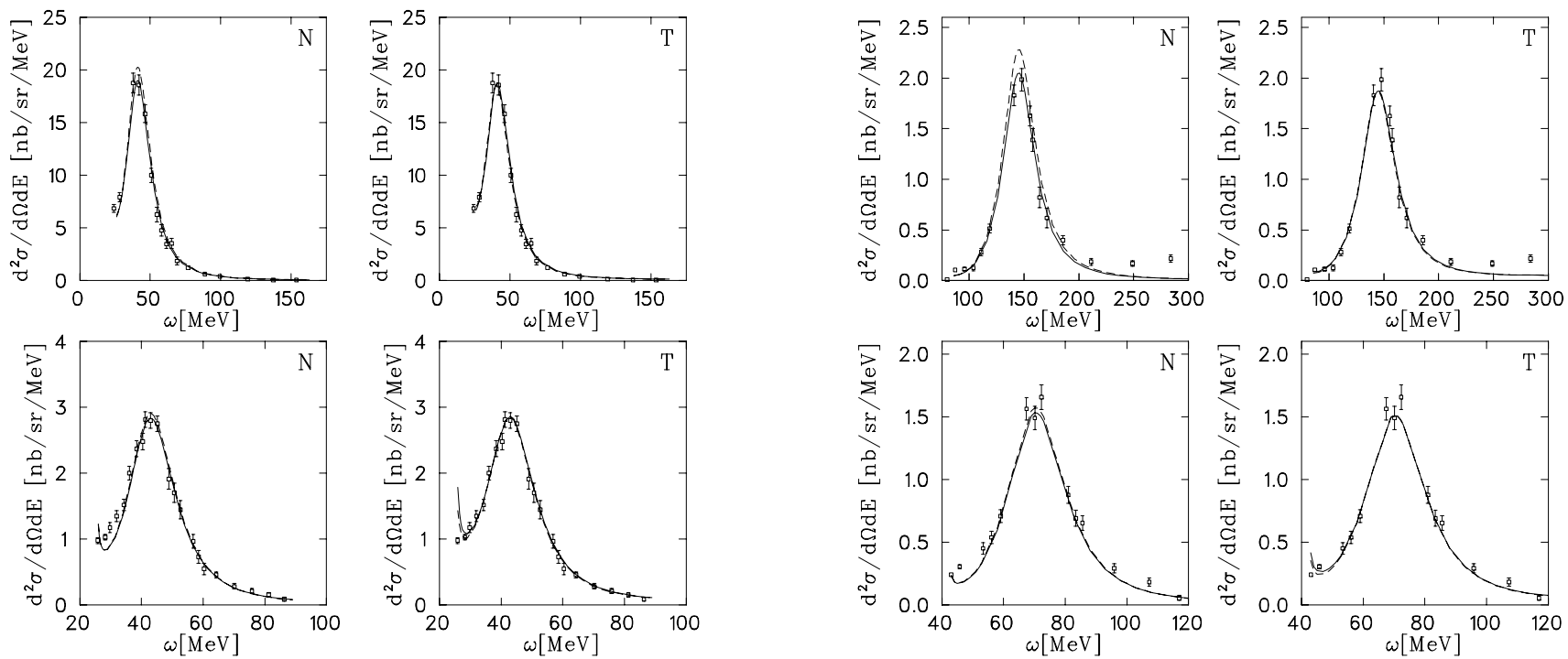

FIG. 15: Inclusive cross sections $d\left(e, e^{\prime}\right)$. Upper panels for $E_{1}^{\text {lab }}=292.8 \mathrm{MeV}$ and $\theta_{e}^{\text {lab }}=60^{\circ}$. Lower panels for $E_{1}^{\text {lab }}=174.3 \mathrm{MeV}$ and $\theta_{e}^{\text {lab }}=134.5^{\circ}$. Exp. from QuINN et al. [59]. Notation of curves: Left panels for nonrelativistic normal theory $(\mathrm{N})$ and right panels for complete theory $(\mathrm{T})$ for BONN-Qb (dashed) and ARGONNE $\mathrm{V}_{18}$ (solid) potentials.

FIG. 16: Inclusive cross sections $d\left(e, e^{\prime}\right)$. Upper panels for $E_{1}^{\text {lab }}=596.8 \mathrm{MeV}$ and $\theta_{e}^{\text {lab }}=60^{\circ}$. Lower panels for $E_{1}^{\mathrm{lab}}=367.7 \mathrm{MeV}$ and $\theta_{e}^{\mathrm{lab}}=134.5^{\circ}$. Exp. from QuINN et al. [59]. Notation of curves: Left panels for nonrelativistic normal theory $(\mathrm{N})$ and right panels for complete theory $(\mathrm{T})$ for BONN-Qb (dashed) and ARGONNE $\mathrm{V}_{18}$ (solid) potentials.

points to a stronger model dependence of $F_{L}$ compared to $F_{T}$. Indeed, this is confirmed by the comparison of the theory with the experimentally determined longitudinal and transverse form factors in Figs. 17 and 18. However, this model dependence disappears almost completely for the full theory.

One should keep in mind that a direct comparison with the response functions $R_{L / T}$ reported in [59] is not possible because of different definitions. However, the relations of $R_{L / T}$ to our form factors is easily obtained by comparing the formal expressions for the inclusive cross section. In Ref. [59] the response functions $R_{L / T}$ are defined by

$$
\frac{d \sigma}{d k_{2}^{\text {lab }} d \Omega_{e}^{\text {lab }}}=\sigma_{\text {Mott }}\left(\xi^{2} R_{L}+\left(\eta+\frac{\xi}{2}\right) R_{T}\right),
$$



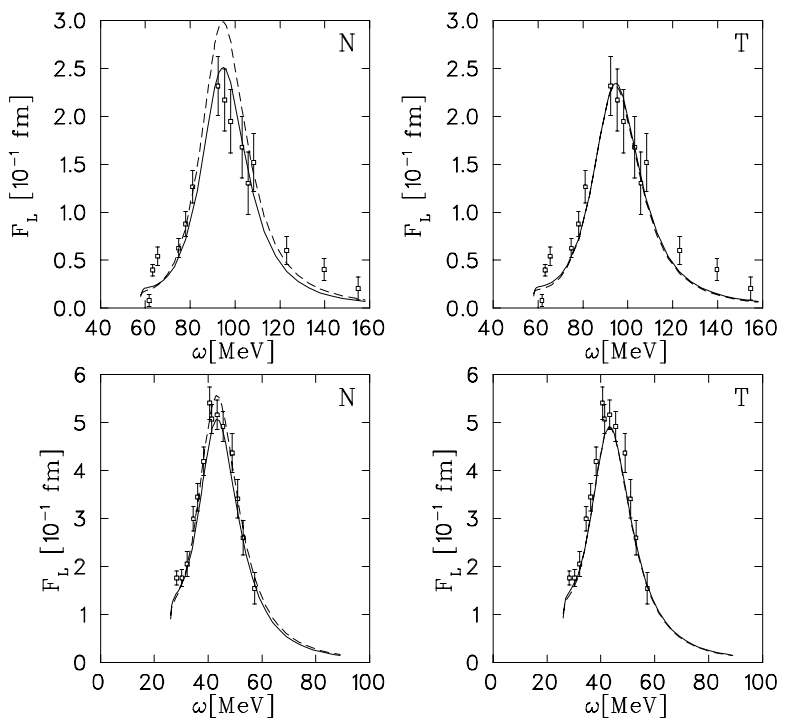

FIG. 17: Longitudinal form factors. Upper panels for $E_{1}^{\text {lab }}=278.5 \mathrm{MeV}$ and $\theta_{e}^{\text {lab }}=134.5^{\circ}$ and lower panels for $E_{1}^{\text {lab }}=174.3 \mathrm{MeV}$ and $\theta_{e}^{\text {lab }}=134.5^{\circ}$. Exp. from QuINN et al. [59]. Notation of curves: Left panels for nonrelativistic normal theory $(\mathrm{N})$ and right panels for complete theory $(\mathrm{T})$ for BONN-Qb (dashed) and ARGONNE V 18 (solid) potentials.
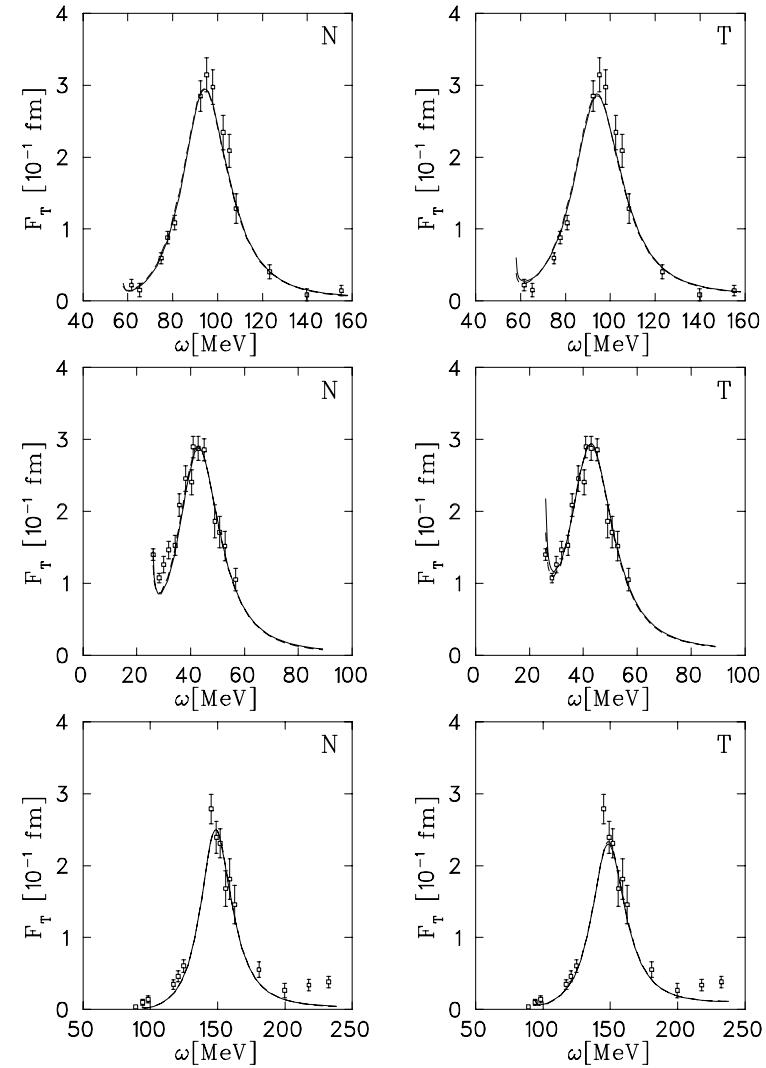

FIG. 18: Transverse form factors. Upper panels for $E_{1}^{\text {lab }}=$ $278.5 \mathrm{MeV}$ and $\theta_{e}^{\text {lab }}=134.5^{\circ}$, middle panels for $E_{1}^{\text {lab }}=$ 174.3 MeV and $\theta_{e}^{\mathrm{lab}}=134.5^{\circ}$, and lower panels for $E_{1}^{\mathrm{lab}}=$ $367.7 \mathrm{MeV}$ and $\theta_{e}^{\text {lab }}=134.5^{\circ}$. Exp. from Quinn et al. [59]. Notation of curves: Left panels for nonrelativistic normal theory $(\mathrm{N})$ and right panels for complete theory $(\mathrm{T})$ for BONN-Qb (dashed) and ARgOnne $\mathrm{V}_{18}$ (solid) potentials.

whereas we use according to (78) in conjunction with (26)

$$
\begin{aligned}
\frac{d \sigma}{d k_{2}^{\text {lab }} d \Omega_{e}^{\text {lab }}} & =6 c\left(k_{1}^{\text {lab }}, k_{2}^{\text {lab }}\right)\left(\rho_{L} F_{L}+\rho_{T} F_{T}\right) \\
& =\sigma_{\text {Mott }}\left(\xi^{2} \frac{\beta^{2} F_{L}}{2 \pi^{2} \alpha}+\left(\eta+\frac{\xi}{2}\right) \frac{F_{T}}{2 \pi^{2} \alpha}\right) .
\end{aligned}
$$

This yields finally

$$
F_{L}=2 \pi^{2} \alpha \frac{q^{2}}{\left(q^{\text {lab }}\right)^{2}} R_{L} \quad \text { and } \quad F_{T}=2 \pi^{2} \alpha R_{T} .
$$

For $F_{L}$ the normal nonrelativistic theory reveals a sizeable potential model dependence according to the left panels of Fig. 17 which, however, is strongly reduced for the complete theory leading to a satisfactory agreement with the data. On the other hand $F_{T}$ in Fig. 18 exhibits much less sensitivity to the potential model for the normal nonrelativistic theory. The improvement by the full calculation in the near threshold region with respect to the data is clearly seen in the middle panels. In the lower panel the absence of pion production in the theory is again responsible for the underestimation above $\omega=200 \mathrm{MeV}$.

\section{B. Exclusive Observables}

Compared to the eight inclusive observables below pion threshold one has 324 independent structure functions for the exclusive case. This greater variety coupled with the angular dependence of each observable allows a more 

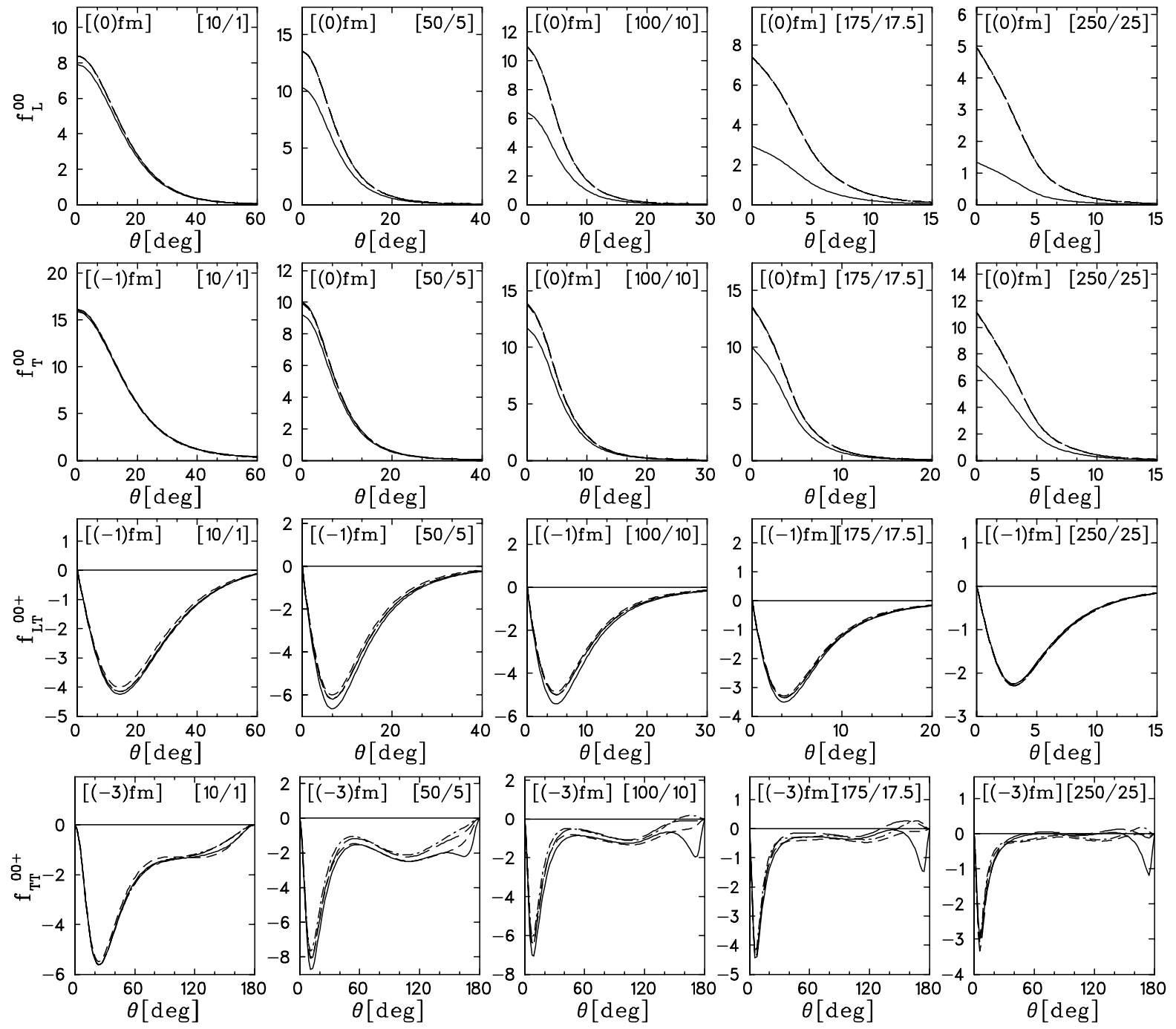

FIG. 19: Survey on the four unpolarized structure functions along the quasi-free ridge, calculated for the BoNN-Qb potential. The top left inset "[(-n) fm]" indicates the unit $\left[10^{-n} \mathrm{fm}\right]$ for the structure function and the top right inset "[ $\left.E_{n p} / q^{2}\right]$ ", where $E_{n p}$ in $[\mathrm{MeV}]$ and $q^{2}$ in $\left[\mathrm{fm}^{-2}\right]$, indicates the kinematic sector. Notation of the curves: N (short dashed), N+MEC (dash-dotted), $\mathrm{N}+\mathrm{MEC}+\mathrm{IC}$ (long dashed), total $=\mathrm{N}+\mathrm{MEC}+\mathrm{IC}+\mathrm{RC}$ (solid).

detailed analysis of the reaction under study. For example, the differential cross section alone provides 41 structure functions if one allows for beam and target polarizations (see Table III). Polarization analysis of one or both outgoing nucleons yields an even larger number of structure functions. However, as has been discussed in detail in Sect. III B 2, in principle a set of 35 independent observables should suffice for a complete determination of all reaction matrix elements. In practice, however, some of these observables will be very difficult to measure experimentally with the required accuracy.

For this reason we have decided to discuss here only those structure functions which are relatively easily accessible, i.e. which require the measurement of only one asymmetry (see Table X of Appendix D). Our discussion will focus on the sensitivity of these structure functions to various interaction effects.

To begin we will briefly give a survey on the four structure functions of the unpolarized differential cross section. Since the structure functions depend on $E_{n p}$ and $q^{2}$ in addition to $\theta^{c . m}$., we have chosen to represent the various kinematical regions by a grid in the $E_{n p}-q^{2}$-plane, defined by $E_{n p}=10,50,100,175,250 \mathrm{MeV}$ and $q^{2}=1,5,10,17.5,25 \mathrm{fm}^{-2}$. Only for $E_{n p}=250 \mathrm{MeV}$ the lowest $q^{2}$-value was taken as $1.5 \mathrm{fm}^{-2}$ because of the photon line according to (7). Fig. 19 shows the four structure functions along the quasi-free ridge. The longitudinal structure function $f_{L}$ shows a pronounced peak in the forward direction essentially caused by the charge interaction of the virtual photon with a proton which is emitted preferentially along $q$. There is no corresponding peak at $180^{\circ}$ for the neutron because of its very small electric form factor. For this reason we have restricted the 

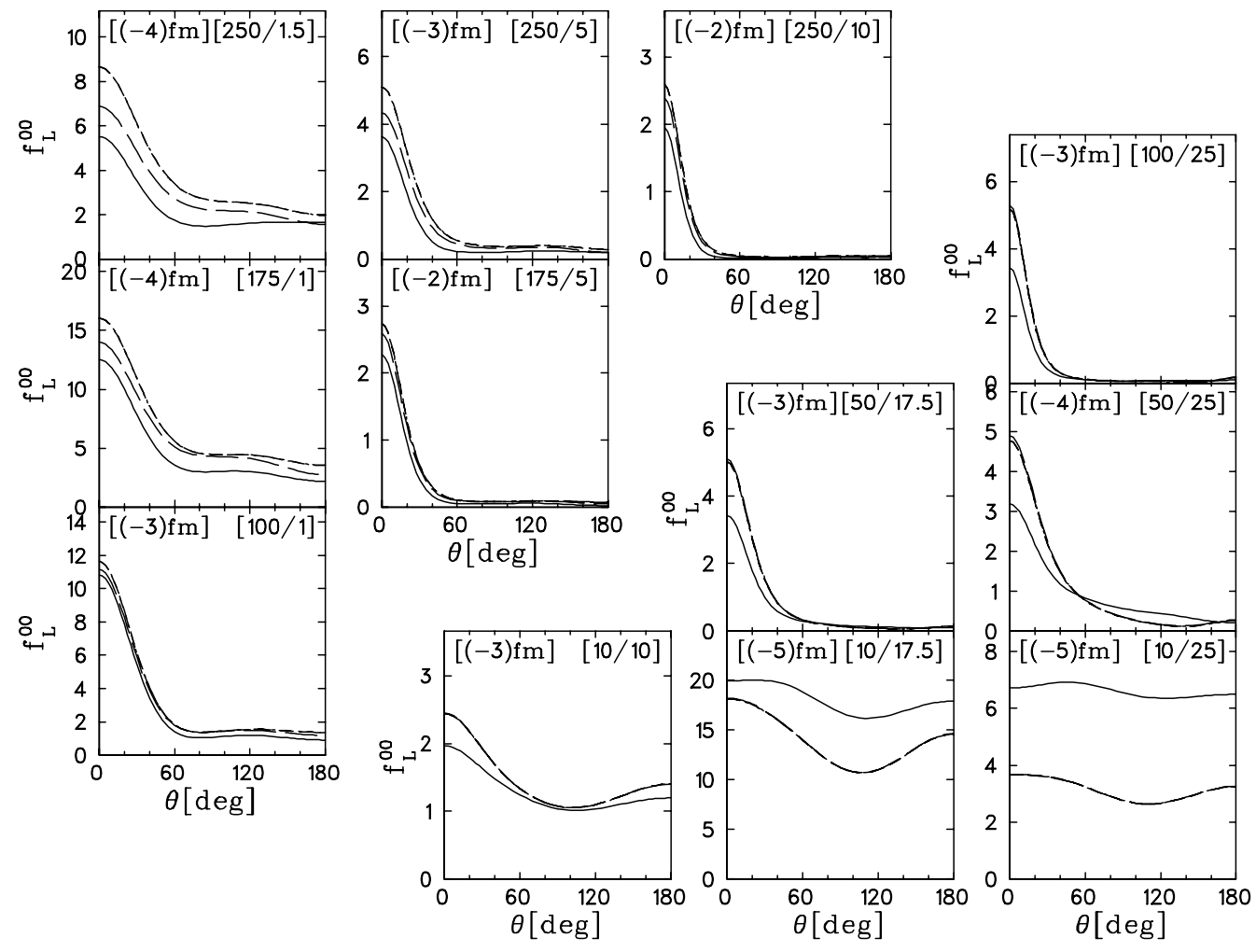

FIG. 20: Survey on longitudinal structure function $f_{L}$ off the quasi-free ridge, calculated for the BonN-Qb potential. Notation of the curves as in Fig. 19.

angular range to the forward direction. With increasing momentum transfer, the width of this forward peak decreases markedly (one should note that the angular range differs for the various cases). Similarly, along the quasi-free ridge $f_{T}$ exhibits forward and backward peaks with decreasing width for growing $q$. The forward peak arises from the e.m. interaction with the dominant spin current of the proton at $\theta=0^{\circ}$ while the backward peak at $\theta=180^{\circ}$ (again not shown) is similar in structure and corresponds to neutron emission along $q$. The ratio of the forward to backward peaks is essentially given by the square of the ratio of the proton to neutron magnetic moments i.e. $\left(\mu_{p} / \mu_{n}\right)^{2} \approx 2$. As was already pointed out in the discussion of the form factors, along the quasi-free ridge most of the interaction effects are marginal, except for relativistic contributions which can result in a decrease of the forward and, in the case of $f_{T}$, backward peaks growing sizeably with increasing momentum transfer. The decrease is particularly significant in $f_{L}$ amounting, for example, at $q^{2}=10 \mathrm{fm}^{-2}$ to about $40 \%$ and at $q^{2}=25 \mathrm{fm}^{-2}$ to even $70 \%$. In $f_{T}$ the effect is smaller, roughly by a factor two.

The interference structure function $f_{L T}$, which has to vanish for $\theta=0^{\circ}$ and $180^{\circ}$, exhibits a negative forward peak of broader distribution than the diagonal structure functions and is almost an order of magnitude lower. A much smaller peak appears for neutron emission close to $180^{\circ}$ with opposite sign which is not shown in Fig. 19. The influence of MEC and IC is very tiny and even RC show up only slightly at intermediate $E_{n p}=50$ to $100 \mathrm{MeV}$. The interference structure function $f_{T T}$ is even smaller, about two orders of magnitude compared to $f_{L T}$. Interaction effects from MEC and IC play only a slightly more significant role. However, RC result in a distinct and sizeable peak at backward angles.

In Figs. 20 through 23 we show the four structure functions for kinematical settings off the quasi-free ridge, i.e. six settings for $E_{n p} / \mathrm{MeV}>10 q^{2} / \mathrm{fm}^{-2}$ in the upper left panels and six settings for $E_{n p} / \mathrm{MeV}<10 q^{2} / \mathrm{fm}^{-2}$ in the lower right panels. Away from the quasi-free ridge, the magnitudes of $f_{L}$ and $f_{T}$ drop rapidly. For $f_{L}$, shown in Fig. 20, the width of the forward peak increases. On the lower right side only RC show quite sizeable influences because of the absence of nonrelativistic MEC contributions and the neglect of a very small charge excitation of the $\Delta-$ for this reason the short and long dashed and the dash-dot curves coincide in Fig. 20 -, whereas on the upper left side, i.e. for $E_{n p} / \mathrm{MeV}>10 q^{2} / \mathrm{fm}^{-2}$, also IC become increasingly important when approaching the $\Delta$-region while keeping the momentum transfer small. The influence of IC arises through the change of the normal wave function component via the dynamic coupling of the $N N$ - and $N \Delta$-channels.

For the transverse structure function $f_{T}$ in Fig. 21, which is slightly larger in size than $f_{L}$, the various interaction 

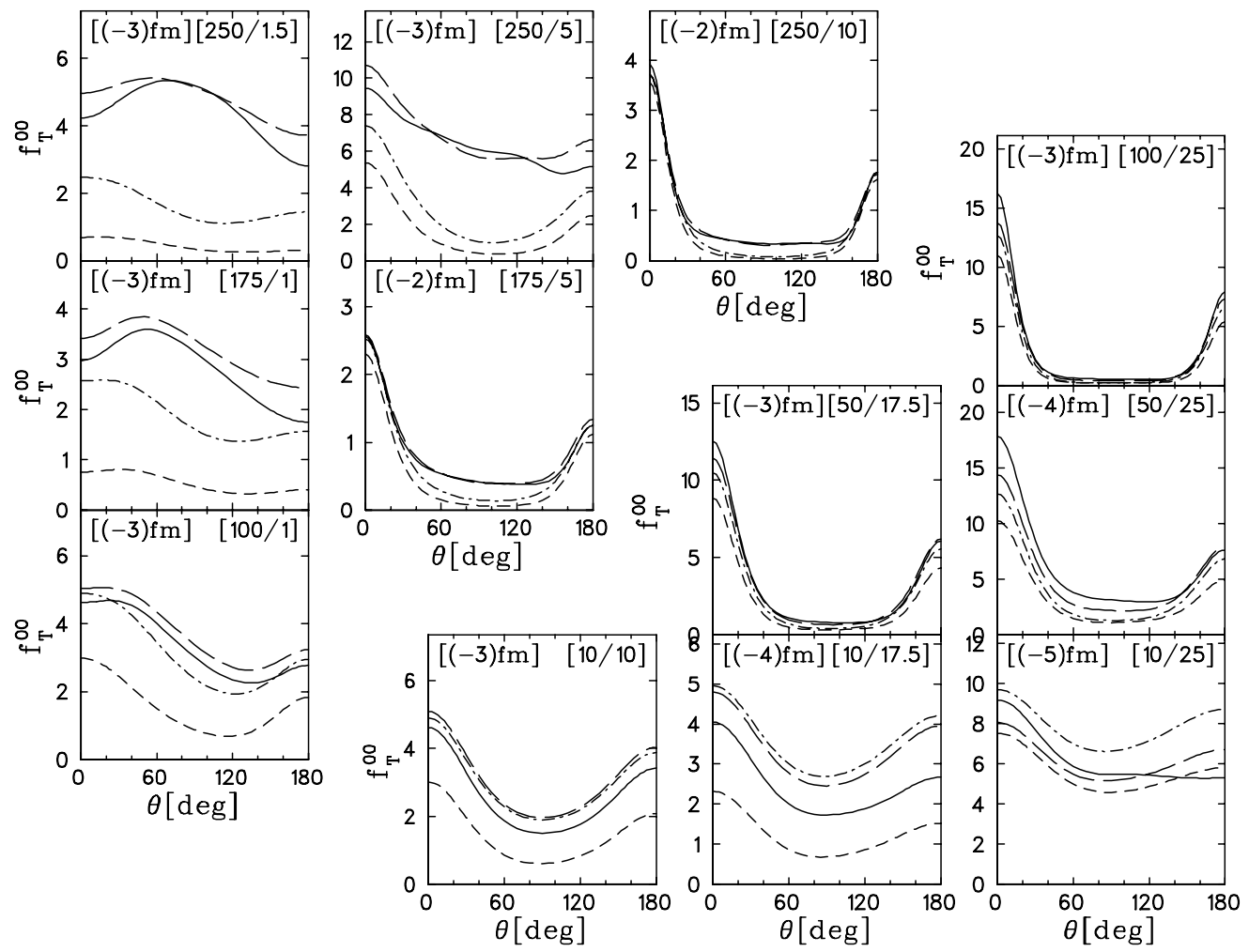

FIG. 21: Survey on transverse structure function $f_{T}$ off the quasi-free ridge, calculated for the BonN-Qb potential. Notation

of the curves as in Fig. 19.
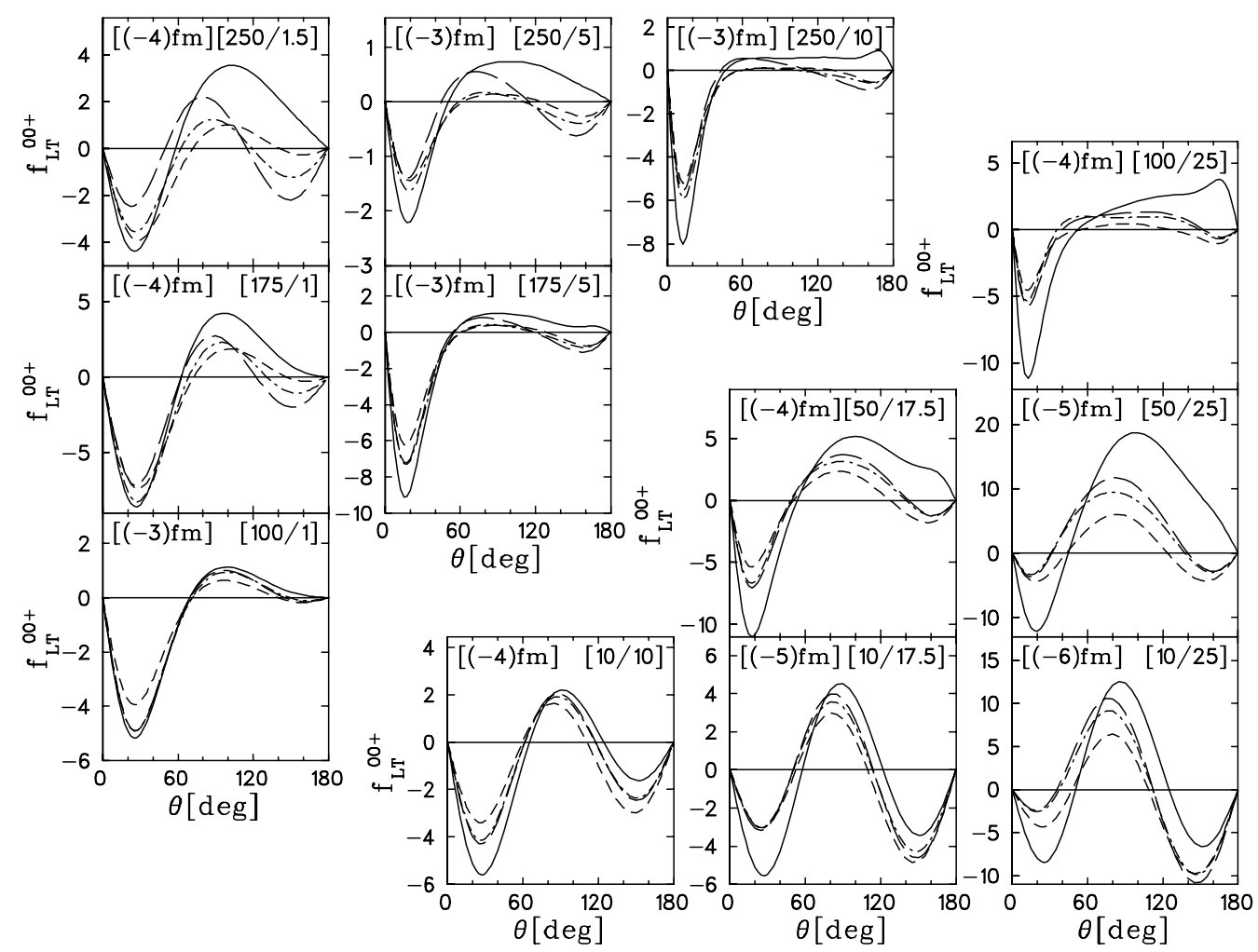

FIG. 22: Survey on interference structure function $f_{L T}$ off the quasi-free ridge, calculated for the BonN-Qb potential. Notation of the curves as in Fig. 19. 

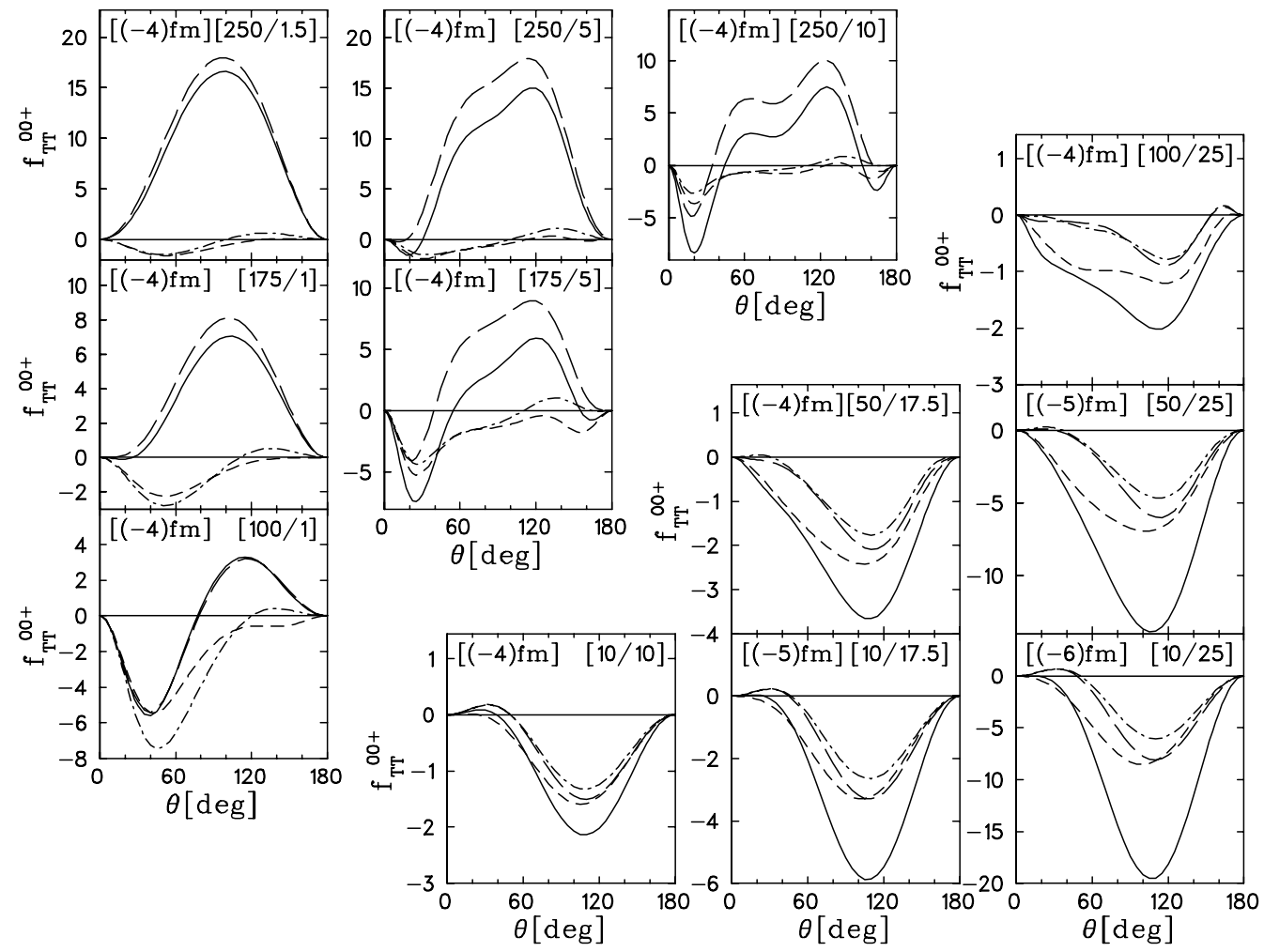

FIG. 23: Survey on interference structure function $f_{T T}$ off the quasi-free ridge, calculated for the BonN-Qb potential. Notation of the curves as in Fig. 19.
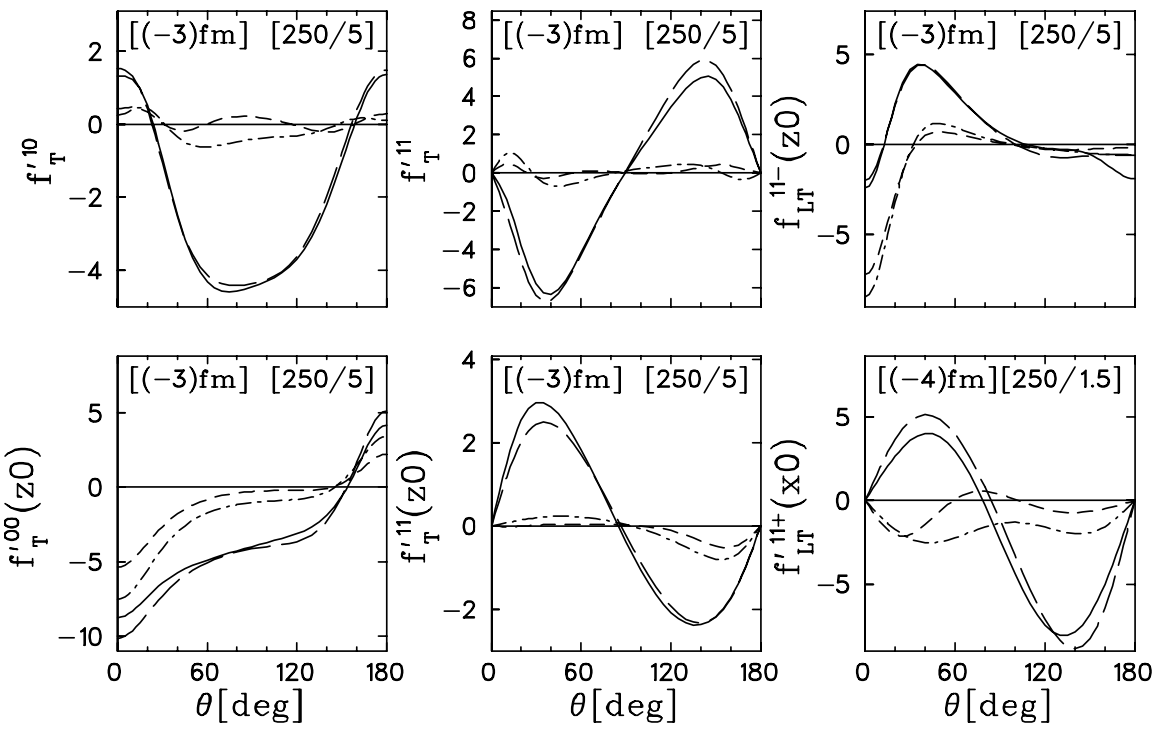

FIG. 24: Influence of IC on various structure functions calculated with the BonN-Qb potential. Notation: N (short dashed), $\mathrm{N}+\mathrm{MEC}$ (dash-dotted), N+MEC+IC (long dashed), T=N+MEC+IC+RC (solid).

effects become much more pronounced off the quasi-free ridge than for $f_{L}$ because of the presence of nonrelativistic MEC and strong transverse excitation of the $\Delta$. Indeed, at low energy MEC provide the largest interaction effect followed by RC while IC remain small. That changes for energies in the region of $\Delta$-excitation above the quasifree ridge, where naturally the IC contributions dominate, although MEC are also sizeable and even RC cannot be neglected, in particular in the forward and backward regions.

The interference structure function $f_{L T}$ in Fig. 22 is comparable in size to $f_{L}$, however, considerably more sensitive 

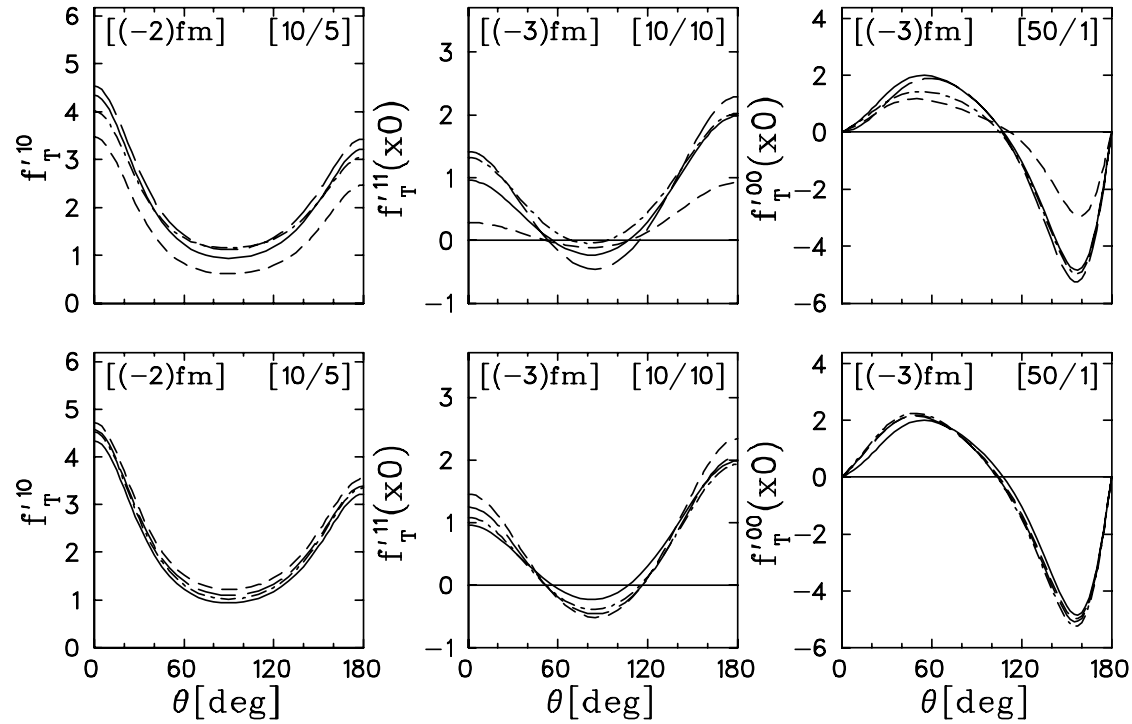

FIG. 25: Upper panels: Influence of MEC on various structure functions calculated with the BonN-Qb potential. Notation as in Fig. 24. Lower panels: Potential model dependence of the total result for the same structure functions. Notation: BonN-R (short dashed), PARIs (dash-dotted), Argonne $V_{18}$ (long dashed), Bonn-Qb (solid).
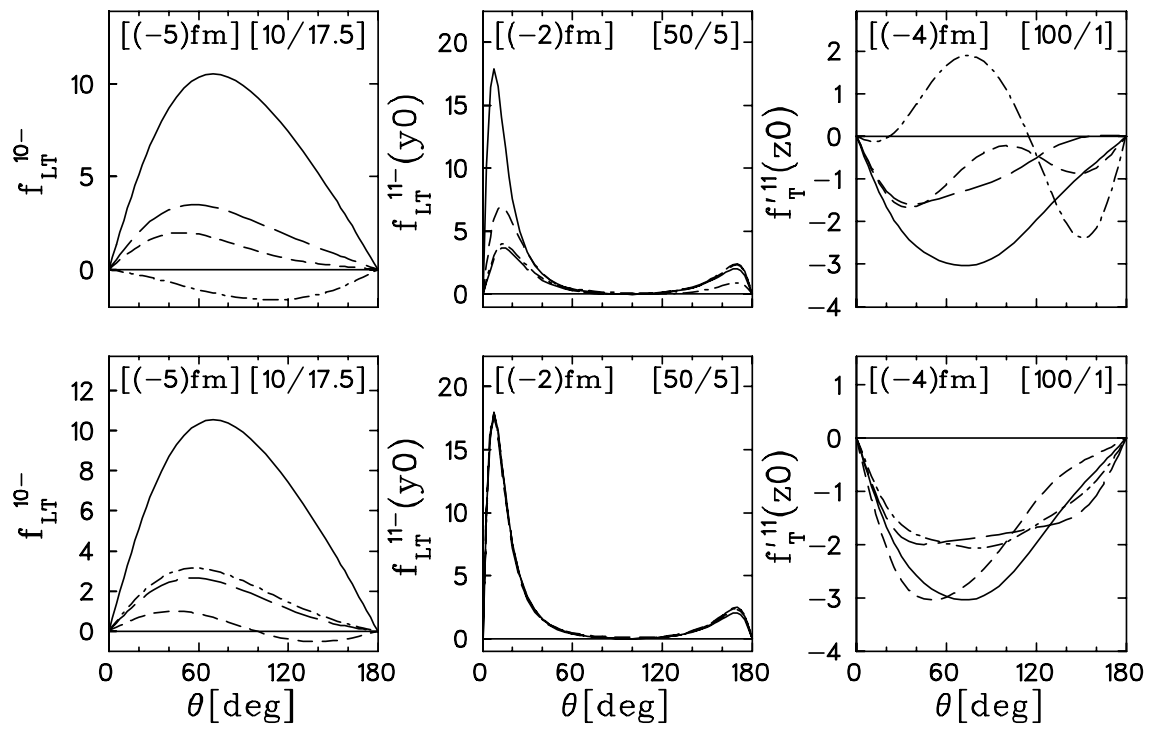

FIG. 26: Upper panels: Influence of RC on various structure functions calculated with the BonN-Qb potential. Lower panels: Potential model dependence of the total result for the same structure functions. Notation as in Fig. 25.

to interaction effects. It shows a negative peak in forward direction, a maximum around $90^{\circ}$ and another but much less pronounced negative peak at backward angles. RC produce by far the strongest effect, deepening the forward negative peak, enhancing the maximum and weakening or even washing out completely the negative backward peak so that a small positive peak results for some kinematics.

The other interference structure function $f_{T T}$, shown in Fig. 23 is of comparable size to $f_{L T}$ off the quasi-free ridge. This is in contrast to their behavior along the quasi-free ridge where $f_{T T}$ is about two orders of magnitude smaller than $f_{L T}$ as depicted in Fig. 19. Below the quasi-free ridge it shows a distinct minimum around $110^{\circ}$ which is decreased by MEC but then deepened slightly by IC and, but more strongly, by RC. Above the quasi-free ridge the influences of interaction effects show in a certain sense an opposite behaviour. Here MEC deepen the minimum, lying more at forward angles, but produce a sign change in the backward direction. This effect is drastically counterbalanced at forward angles by IC and amplified at larger angles. The additional RC lead then finally to a smaller reduction, deepening the small forward minimum, which exists for higher $E_{n p}$ and $q^{2}$. 

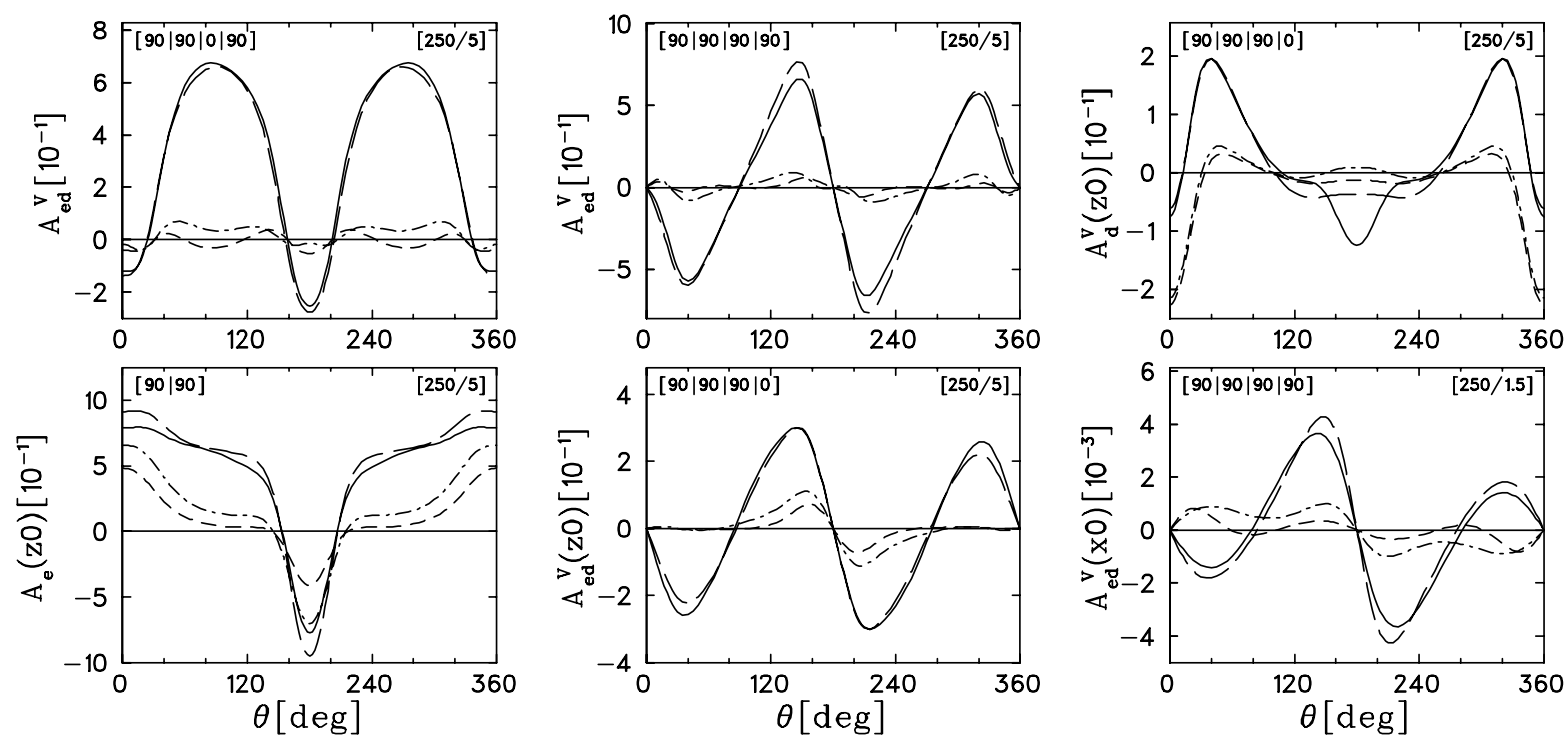

FIG. 27: Influence of IC on asymmetries for kinematic settings for the separation of the various structure functions in Fig. 24 calculated with the BonN-Qb potential. Notation: N (short dashed), N+MEC (dash-dotted), N+MEC+IC (long dashed), $\mathrm{T}=\mathrm{N}+\mathrm{MEC}+\mathrm{IC}+\mathrm{RC}$ (solid). The upper left inset lists the various angles according to $\left[\theta_{e}, \phi, \theta_{d}, \phi_{d}\right]$.

Now we turn to the question of which structure functions exhibit the most significant sensitivity with respect to the various interaction effects. As already mentioned we take into account only those cases which require a single asymmetry measurement (see Table X of Appendix D). In addition, we consider as observables the cross sections and single proton polarizations $(x 0, y 0, z 0)$ only. We have studied these selected structure functions in the same kinematic regions as taken for the survey of the unpolarized structure functions but present here only those kinematic cases where we found the strongest signatures.

In Fig. 24 we show the most relevant cases for the IC contribution. It is not surprising that they are all found at an excitation energy in the $\Delta$-resonance region at lower momentum transfer. As pointed out before, appropriate potential models with $\Delta$ degrees of freedom are lacking and thus we only show results for the coupled channel calculation with the renormalized BONN-Qb potential. The figure shows that there are quite a number of different structure functions which are essentially dominated by their IC contribution and thus are ideal cases for the study of such IC effects. It it worthwhile to note that there are not only transverse $\left(f_{T}^{\prime 10}, f_{T}^{\prime 11}, f_{T}^{\prime 00}(z 0), f_{T}^{\prime 11}(z 0)\right)$, but also LT-type structure functions $\left(f_{L T}^{\prime 11-}(z 0), f_{L T}^{\prime 11+}(x 0)\right)$ among the cases presented.

Observables which exhibit sensitivity to the MEC contribution are shown in Fig. 25. While for $f_{T}^{\prime 10}$ the strongest relative MEC effect is found around $\theta=90^{\circ}$, for $f_{T}^{\prime 11}(x 0)$ and $f_{T}^{\prime 00}(x 0)$ one has the most pronounced effects at backward angles. The potential model dependence is not very important and is particularly small for $f_{T}^{\prime 00}(x 0)$. We would like to mention that the $f_{T}^{\prime 10}$ result is very similar to that of $f_{T}^{00}$ for the same kinematics (see Fig. 21). In fact, $f_{T}^{00}$ is very sensitive to the MEC contribution and can in principle be determined in a single measurement (scattered electron at backward angle and $\phi=45^{\circ}$ ).

In Fig. 26 we show those structure functions where there are major effects due to relativistic contributions. A very strong effect is seen for $f_{L T}^{10-}$, although this structure function shows a rather severe potential model dependence as well. For $f_{T}^{\prime 11}(z 0)$ one finds a similar situation, but with somewhat smaller relativistic influence and a less pronounced variation with the potential model. On the contrary, for $f_{L T}^{11-}(y 0)$ one observes essentially no potential model dependence and a large relativistic contribution at forward angles.

In view of the fact that, besides the unpolarized differential cross section, the quantities which one measures experimentally are asymmetries, we present in Figs. 27 through 29 the asymmetries corresponding to the structure functions of Figs. 24 through 26 in order to see which of them produce sizeable, i.e. easily accessible asymmetries. In order to emphasize the interaction and relativistic effects in the numerator, the following asymmetries always refer to the unpolarized differential cross section in which all contributions from MEC, IC and RC are included, i.e. $S_{0}$ in Eq. (83) is $S_{0}(T)$.

With respect to IC effects, one readily notes in Fig. 27 three sizeable asymmetries of the order of one, namely $A_{\text {ed }}^{V}$ for two different settings $\left(\theta_{d}=0^{\circ}\right.$ and $90^{\circ}$ with $\left.\phi_{d}=90^{\circ}\right)$, requiring electron and deuteron vector polarization, and $A_{e}(z 0)$, requiring electron polarization and a proton polarimeter. Two other asymmetries, $A_{d}^{V}(z 0)$ and $A_{e d}^{V}(z 0)$, are of the order of 0.2 . Only $A_{e d}^{V}(x 0)$ is too small being of the order of 0.004 . Of the asymmetries sensitive to MEC in 

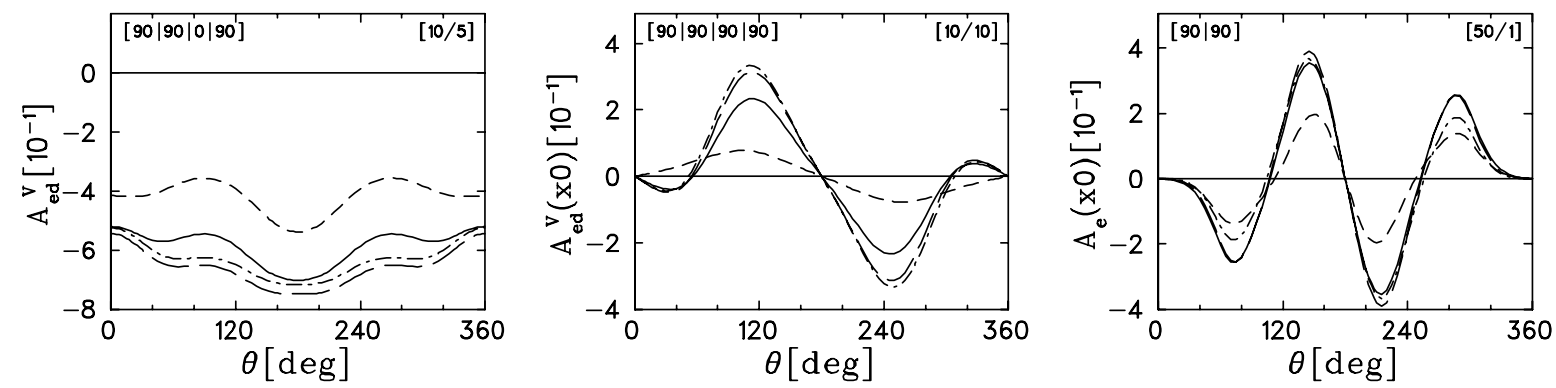

FIG. 28: Influence of MEC on asymmetries for kinematic settings for the separation of the various structure functions in Fig. 25 calculated with the BonN-Qb potential. Notation as in Fig. 27.
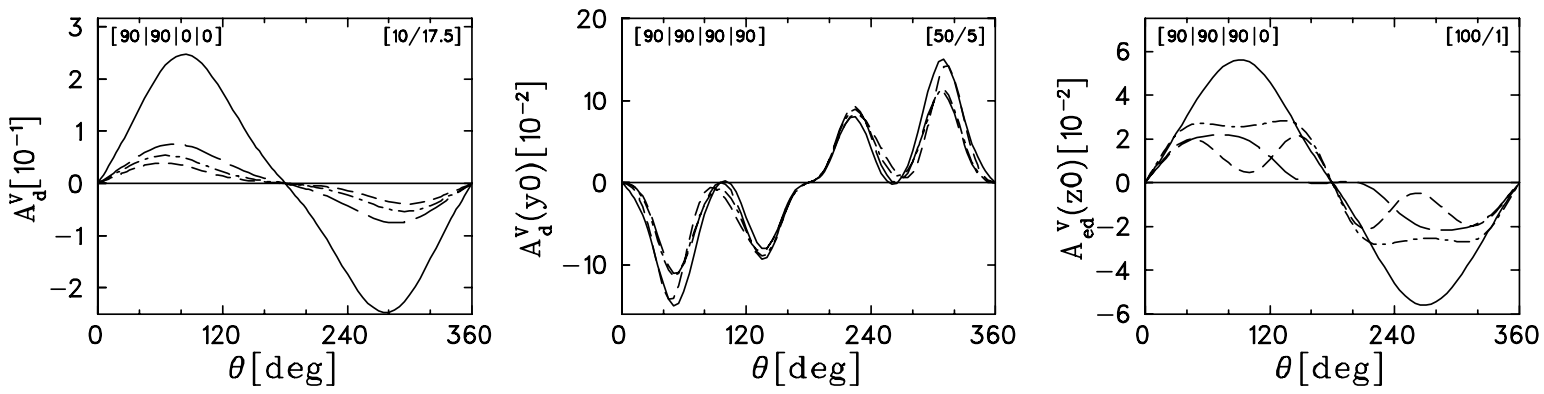

FIG. 29: Influence of RC on asymmetries for kinematic settings for the separation of the various structure functions in Fig. 26 calculated with the BonN-Qb potential. Notation as in Fig. 27.

Fig. 28 the largest one is $A_{e d}^{V}$ being of the order of 0.7 , whereas the other two are smaller, of the order of 0.4. With respect to RC, two asymmetries in Fig. 29 are sufficiently large, namely $A_{d}^{V}$ and $A_{d}^{V}(y 0)$ with a magnitude of about 0.1 to 0.2 , but only $A_{d}^{V}$ exhibits a large RC effect.

Finally we would like to briefly discuss irrespective of the problem of separating a specific structure function the question which observables show in specific asymmetries of sizeable magnitude either a large influence or none by interaction contributions. We have investigated for a large number of observables various asymmetries for a variety of kinematic settings. Some results of this search are presented in Figs. 30 and 31.

Fig. 30 exhibits the tensor asymmetry $A_{d}^{T}$ for two different settings of the angles $\phi, \theta_{d}$ and $\phi_{d}$. Without interaction effects the asymmetry would be quite small, about 0.1 or less. In the left panel, MEC lead to a strong enhancement around $0^{\circ}$ and $180^{\circ}$ which are partially canceled by relativistic contributions. On the other hand, IC show a large and dominant influence around $90^{\circ}$ and $270^{\circ}$ where the other effects are very small. The right panel is an instructive example on how the the asymmetry and the relative size of the various contributions change with a change of the out-of-plane angle $\phi$ and the deuteron orientation angles. In the left panel of Fig. 31, the vector asymmetry $A_{\text {ed }}^{V}$ exhibits at low energy but higher momentum transfer a large MEC effect which is cut down to almost one half by relativistic contributions whereas IC is negligible. The same asymmetry at a different kinematics, $E_{n p}=100 \mathrm{MeV}$ and $q=1 \mathrm{fm}^{-2}$, in the right panel of Fig. 31 shows a strong influence from MEC and IC of almost equal size and
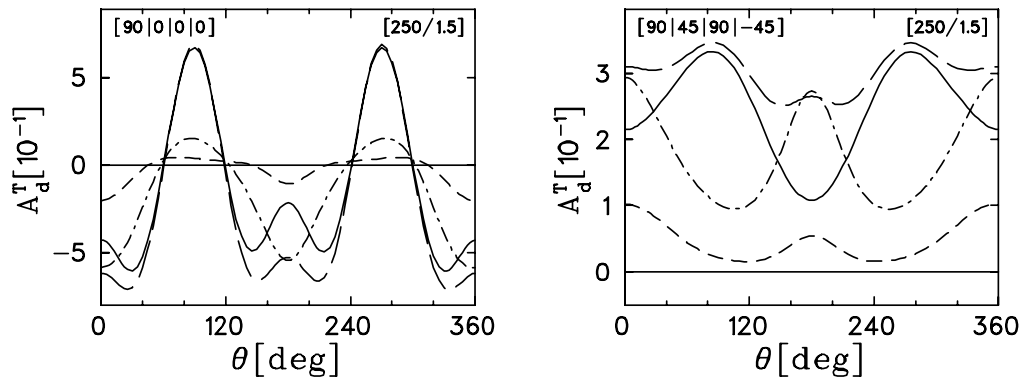

FIG. 30: Tensor asymmetry $A_{d}^{T}$ for $E_{n p}=250 \mathrm{MeV}, q^{2}=1.5 \mathrm{fm}^{-2}$ calculated with the BonN-Qb potential. Notation as in Fig. 27. 

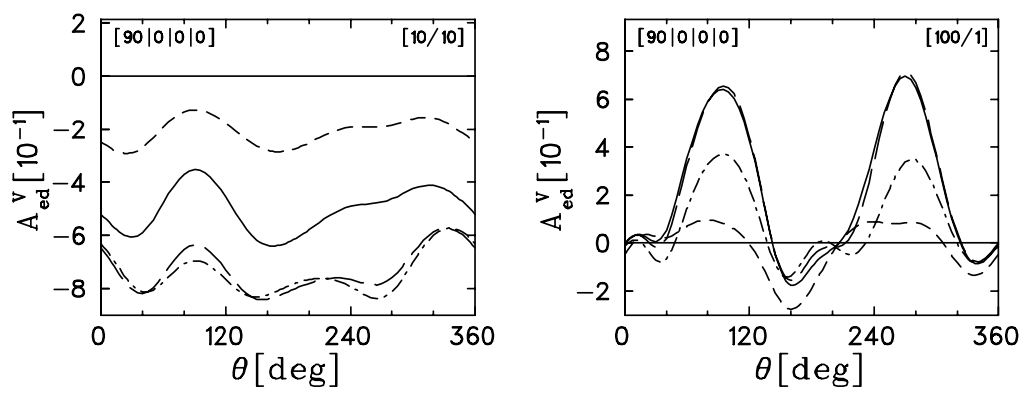

FIG. 31: Vector asymmetry $A_{e d}^{V}$ at two different off-quasi-free kinematics, calculated with the BonN-Qb potential. Left panel for $E_{n p}=10 \mathrm{MeV}, q^{2}=10 \mathrm{fm}^{-2}$ and right panel for $E_{n p}=100 \mathrm{MeV}, q^{2}=1 \mathrm{fm}^{-2}$. Notation as in Fig. 27 .
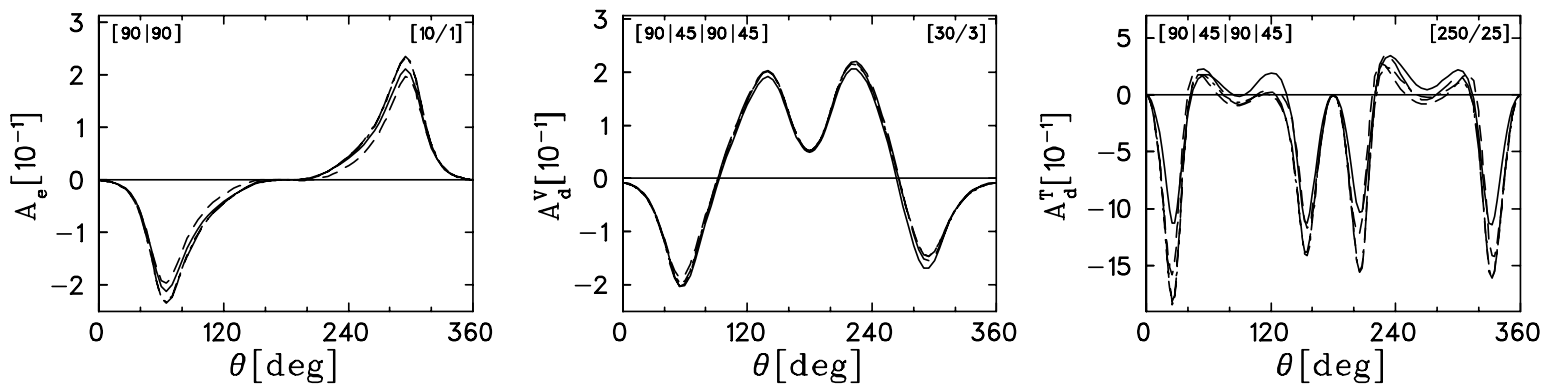

FIG. 32: Asymmetries at three different on-quasi-free kinematics, calculated with the BonN-Qb potential. Left panel $A_{e}$ for $E_{n p}=10 \mathrm{MeV}, q^{2}=1 \mathrm{fm}^{-2}$, middle panel $A_{d}^{V}$ for $E_{n p}=30 \mathrm{MeV}, q^{2}=3 \mathrm{fm}^{-2}$, and right panel $A_{d}^{T}$ for $E_{n p}=250 \mathrm{MeV}$, $q^{2}=25 \mathrm{fm}^{-2}$. Notation as in Fig. 27 .

interfering constructively, while $\mathrm{RC}$ effects are tiny.

As last examples we show in Fig. 32 three asymmetries for on-quasi-free kinematics, two at low energies and one in the $\Delta$-region which exhibit a nearly interaction independent behaviour. The two asymmetries at quite low energies, $A_{e}$ in the left panel and in particular $A_{d}^{V}$ in the middle panel show very little influences from interaction and relativistic effects. In general these effects increase with growing energy $E_{n p}$. But even for the example in the right panel at considerably high energy such influences are still relatively small. It is not surprising, that one finds such a behaviour for kinematics belonging to the quasi-free ridge, because one expects that influences from FSI, MEC and IC will be minimal there. Because of their model independence, such cases provide consistency checks for theory and experiment.

With this we will close the discussion of our results. We will not compare to experimental data on the exclusive reaction [61-75] because they have already been compared to our approach and thus nothing new can be said.

\section{SUMMARY AND CONCLUSION}

In this work we have presented a thorough and detailed survey on polarization observables in electrodisintegration of the deuteron. It contains a general review of the basic formal aspects with respect to kinematics, the definition of observables and structure functions, their multipole decomposition, the question of completeness and the construction of independent sets of observables including analytic solutions of the $t$-matrix elements in terms of structure functions.

Furthermore, a detailed account of the dynamic ingredients of the theoretical framework is given with respect to the basic $N N$-force, the associated meson exchange currents, isobar configurations for the consideration of internal nucleon dynamics, and leading order relativistic contributions.

As results we first have presented a general survey on the unpolarized and polarized form factors of the inclusive process indicating the various kinematic regions with respect to energy and momentum transfer where sensitivities to MEC, IC and RC according to the theoretical framework are to be expected. Furthermore, a comparison with experimental data for the near threshold region and for quasi-free kinematics is discussed. For the threshold region the influence of interaction effects is quantitatively confirmed. Also the quasi-free peak is well reproduced provided the reduction by relativistic contributions is included.

For the exclusive reaction an extensive survey on the unpolarized structure function for a representative grid of energy and momentum transfers is given including a detailed discussion of the various interaction effects. Furthermore, for a selected set of polarization structure functions whose determination requires only one asymmetry measurement, 
we have chosen those kinematic regions in which either IC or MEC or RC play a major role with subsequent discussion of the associated asymmetries.

Finally we hope that this survey will stimulate further experimental and theoretical research allowing one to test more thoroughly the underlying basic dynamics. This is of particular importance with respect to the question how well do we understand the strong interaction in terms of effective degrees of freedom, i.e. in terms of meson, nucleon and isobar degrees of freedom, and where will we need to introduce explicitly the basic quark and gluon degrees of freedom of QCD.

\section{Acknowledgments}

This work was supported by the Deutsche Forschungsgemeinschaft (SFB 443) and by the National Science and Engineering Research Council of Canada.

\section{APPENDIX A: EXPLICIT EXPRESSIONS FOR THE $\mathcal{U}_{X}^{\lambda^{\prime} \lambda I M}$ IN THE STANDARD REPRESENTATION OF THE $t$-MATRICES}

In view of the angular momentum algebra, it is useful to switch to a spherical representation replacing the cartesian components of the nucleon spin operators by their spherical ones

$$
\sigma_{\alpha}(i)=\sum_{\tau=0,1} \sum_{\nu=-\tau}^{\tau} s_{\alpha}^{\tau \nu} \sigma_{\nu}^{[\tau]}(i) \quad \text { for } \alpha=0, \ldots, 3,
$$

where we have introduced the equivalent complete set of $2 \times 2$-matrices in $s=1 / 2$ space

$$
\sigma_{\nu}^{[\tau]}(i) \quad \text { for } \tau=0,1 \text { and }|\nu| \leq \tau,
$$

defining $\sigma_{0}^{[0]}=\sigma_{0}=\mathbb{1}_{2}$ and $\sigma_{\nu}^{[1]}=\sigma_{\nu}$, the spherical components of the Pauli spin matrices. The transformation matrix is given by

$$
s_{\alpha}^{\tau \nu}=\bar{c}(\alpha) \delta_{\tau, \widetilde{\tau}(\alpha)}\left(\delta_{\nu, \widetilde{\nu}(\alpha)}+\hat{c}(\alpha) \delta_{\nu,-\widetilde{\nu}(\alpha)}\right)
$$

with

$$
\begin{aligned}
& \hat{c}(\alpha)=\delta_{\alpha 2}-\delta_{\alpha 1}, \quad \bar{c}(\alpha)=\left\{\begin{array}{cc}
1 & \text { for } \alpha=0,3 \\
\frac{i^{-\alpha-1}}{\sqrt{2}} \text { for } \alpha=1,2
\end{array},\right. \\
& \widetilde{\tau}(\alpha)=1-\delta_{\alpha 0}, \quad \widetilde{\nu}(\alpha)=\left\{\begin{array}{ll}
0 & \text { for } \alpha=0,3 \\
1 & \text { for } \alpha=1,2
\end{array} .\right.
\end{aligned}
$$

For the inverse transformation from spherical to cartesian components one easily finds

$$
\sigma_{\nu}^{[\tau]}(i)=\sum_{\alpha=0}^{3} c_{\tau \nu}^{\alpha} \sigma_{\alpha}(i),
$$

where we have introduced

$$
c_{\tau \nu}^{\alpha}=c(\nu)\left(\delta_{\alpha, a(\tau, \nu)}+i \nu \delta_{\alpha, b(\tau, \nu)}\right)
$$

and

$$
c(\nu)=-\frac{\nu}{\sqrt{2}} \delta_{|\nu| 1}+\delta_{\nu 0}, \quad a(\tau, \nu)=3 \tau-2|\nu|, \quad b(\tau, \nu)=3 \tau-|\nu| .
$$

Then the transformation of the $\mathcal{U}$ 's to spherical components is given by

$$
\mathcal{U}_{\alpha^{\prime} \alpha}^{\lambda^{\prime} \lambda I M}=\sum_{\tau^{\prime} \nu^{\prime} \tau \nu} s_{\alpha^{\prime}}^{\tau^{\prime} \nu^{\prime}} s_{\alpha}^{\tau \nu} \mathcal{U}_{\tau^{\prime} \nu^{\prime} \tau \nu}^{\lambda^{\prime} \lambda I M}
$$


where $\mathcal{U}_{\tau^{\prime} \nu^{\prime} \tau \nu}^{\lambda^{\prime} \lambda I M}$ is defined as in (51) with $\sigma_{x_{j}}(i)$ being replaced by the spherical components according to (A2). In the coupled representation one has the following explicit form for the $\mathcal{U}$ 's

$$
\mathcal{U}_{\tau^{\prime} \nu^{\prime} \tau \nu}^{\lambda^{\prime} \lambda I M}=\sum_{S \sigma}(-)^{\tau^{\prime}+\tau+\sigma} \hat{\tau}^{\prime} \hat{\tau} \hat{S}\left(\begin{array}{ccc}
\tau^{\prime} & \tau & S \\
\nu^{\prime} & \nu & -\sigma
\end{array}\right) V_{\lambda^{\prime} \lambda I M}^{\tau^{\prime} \tau S \sigma}
$$

where the quantities $V_{\lambda^{\prime} \lambda I M}^{\tau^{\prime} \tau S \sigma}$ are given by

$$
V_{\lambda^{\prime} \lambda I M}^{\tau^{\prime} \tau S \sigma}=2 \hat{S} \sum_{s^{\prime} s} \hat{s}^{\prime} \hat{s}\left\{\begin{array}{ccc}
\frac{1}{2} & \frac{1}{2} & \tau^{\prime} \\
\frac{1}{2} & \frac{1}{2} & \tau \\
s^{\prime} & s & S
\end{array}\right\} u_{\lambda^{\prime} \lambda I M}^{s^{\prime} s S \sigma}
$$

with

$$
u_{\lambda^{\prime} \lambda I M}^{s^{\prime} S S \sigma}=\hat{I} \sqrt{3} \sum_{m_{s}^{\prime} m_{s} m^{\prime} m}(-)^{1-m+s^{\prime}-m_{s}^{\prime}}\left(\begin{array}{ccc}
1 & 1 & I \\
m^{\prime} & -m & M
\end{array}\right)\left(\begin{array}{ccc}
s^{\prime} & s & S \\
m_{s}^{\prime} & -m_{s} & -\sigma
\end{array}\right) t_{s^{\prime} m_{s}^{\prime} \lambda^{\prime} m^{\prime}}^{*} t_{s m_{s} \lambda m} .
$$

Specifying the observable $X$, one has in detail:

(i) differential cross section $(X=1=(00))$

$$
\mathcal{U}_{1}^{\lambda^{\prime} \lambda I M}=\sum_{s} \hat{s} u_{\lambda^{\prime} \lambda I M}^{s s 00}
$$

(ii) single nucleon polarization $(X=(i 0)$ for the proton or $X=(0 i)$ for the neutron)

$$
\begin{aligned}
& \mathcal{U}_{x_{p}}^{\lambda^{\prime} \lambda I M}=-\sqrt{\frac{3}{2}}\left(V_{\lambda^{\prime} \lambda I M}^{1011}-V_{\lambda^{\prime} \lambda I M}^{101-1}\right), \\
& \mathcal{U}_{x_{n}}^{\lambda^{\prime} \lambda I M}=-\sqrt{\frac{3}{2}}\left(V_{\lambda^{\prime} \lambda I M}^{0111}-V_{\lambda^{\prime} \lambda I M}^{011-1}\right), \\
& \mathcal{U}_{y_{p}}^{\lambda^{\prime} \lambda I M}=i \sqrt{\frac{3}{2}}\left(V_{\lambda^{\prime} \lambda I M}^{1011}+V_{\lambda^{\prime} \lambda I M}^{101-1}\right), \\
& \mathcal{U}_{y_{n}}^{\lambda^{\prime} \lambda I M}=i \sqrt{\frac{3}{2}}\left(V_{\lambda^{\prime} \lambda I M}^{0111}+V_{\lambda^{\prime} \lambda I M}^{011-1}\right) \\
& \mathcal{U}_{z_{p}}^{\lambda^{\prime} \lambda I M}=\sqrt{3} V_{\lambda^{\prime} \lambda I M}^{1010}, \\
& \mathcal{U}_{z_{n}}^{\lambda^{\prime} \lambda I M}=\sqrt{3} V_{\lambda^{\prime} \lambda I M}^{0110},
\end{aligned}
$$

where

$$
\begin{aligned}
& V_{\lambda^{\prime} \lambda I M}^{101 \sigma}=\sqrt{2} \sum_{s^{\prime} s}(-)^{s} \hat{s}^{\prime} \hat{s}\left\{\begin{array}{lll}
s^{\prime} & s & 1 \\
\frac{1}{2} & \frac{1}{2} & \frac{1}{2}
\end{array}\right\} u_{\lambda^{\prime} \lambda I M}^{s^{\prime} s 1 \sigma}, \\
& V_{\lambda^{\prime} \lambda I M}^{011 \sigma}=\sqrt{2} \sum_{s^{\prime} s}(-)^{s^{\prime}} \hat{s}^{\prime} \hat{s}\left\{\begin{array}{lll}
s^{\prime} & s & 1 \\
\frac{1}{2} & \frac{1}{2} & \frac{1}{2}
\end{array}\right\} u_{\lambda^{\prime} \lambda I M}^{s^{\prime} s 1 \sigma} .
\end{aligned}
$$

(iii) double nucleon polarization $(X=(i j))$

$$
\begin{aligned}
\mathcal{U}_{x x / y y}^{\lambda^{\prime} \lambda I M} & =-\sqrt{3}\left[V_{\lambda^{\prime} \lambda I M}^{1100}+\frac{1}{\sqrt{2}} V_{\lambda^{\prime} \lambda I M}^{1120} \mp \frac{\sqrt{3}}{2}\left(V_{\lambda^{\prime} \lambda I M}^{1122}+V_{\lambda^{\prime} \lambda I M}^{112-2}\right)\right] \\
\mathcal{U}_{z z}^{\lambda^{\prime} \lambda I M} & =-\sqrt{3}\left[V_{\lambda^{\prime} \lambda I M}^{1100}-\sqrt{2} V_{\lambda^{\prime} \lambda I M}^{1120}\right] \\
\mathcal{U}_{x y / y x}^{\lambda^{\prime} \lambda I M} & =-\frac{3 i}{2}\left[ \pm \sqrt{2} V_{\lambda^{\prime} \lambda I M}^{1110}+\left(V_{\lambda^{\prime} \lambda I M}^{1122}-V_{\lambda^{\prime} \lambda I M}^{112-2}\right)\right] \\
\mathcal{U}_{x z / z x}^{\lambda^{\prime} \lambda I M} & =-\frac{3}{2}\left[ \pm\left(V_{\lambda^{\prime} \lambda I M}^{1111}+V_{\lambda^{\prime} \lambda I M}^{111-1}\right)+\left(V_{\lambda^{\prime} \lambda I M}^{1121}-V_{\lambda^{\prime} \lambda I M}^{112-1}\right)\right] \\
\mathcal{U}_{y z / z y}^{\lambda^{\prime} \lambda I M} & =\frac{3 i}{2}\left[ \pm\left(V_{\lambda^{\prime} \lambda I M}^{1111}-V_{\lambda^{\prime} \lambda I M}^{111-1}\right)+\left(V_{\lambda^{\prime} \lambda I M}^{1121}+V_{\lambda^{\prime} \lambda I M}^{112-1}\right)\right]
\end{aligned}
$$




\section{APPENDIX B: QUADRATIC RELATIONS BETWEEN OBSERVABLES}

In this appendix we will show that for a set of $n$ independent $t$-matrix elements $\left\{t_{j} ; j=1 \ldots n\right\}$ one finds exactly $(n-1)^{2}$ quadratic relations between observables by which the $n^{2}$ linearly independent observables are reduced to a set of $2 n-1$ independent ones. To this end we introduce the bilinear form in the $t$-matrix elements

$$
T_{j^{\prime} j}=t_{j^{\prime}}^{*} t_{j}
$$

which can be expressed as a linear form of the observables $\mathcal{O}^{\alpha}$

$$
T_{j^{\prime} j}=\sum_{\alpha} \tau_{j^{\prime} j}^{\alpha} \mathcal{O}^{\alpha}
$$

with appropriate coefficients $\tau_{j^{\prime} j}^{\alpha}$. They have the property

$$
\tau_{j^{\prime} j}^{\alpha}=\tau_{j j^{\prime}}^{\alpha *},
$$

which follows from $T_{j^{\prime} j}=T_{j j^{\prime}}^{*}$ and the fact that the observables are real quantities. It is straightforward to show that these bilinear forms obey the relation

$$
T_{j^{\prime} j} T_{l m}=T_{j^{\prime} m} T_{l j},
$$

which, expressed in terms of observables, yields quadratic relations between the latter. In particular, choosing $k=$ $l=m$, one finds

$$
T_{j^{\prime} j}=\frac{T_{j^{\prime} k} T_{k j}}{T_{k k}},
$$

where $k$ can be chosen arbitrarily. It is also clear that from (B5) one can recover the relation (B4). Thus we only need to consider the latter relation, and the question is, how many independent quadratic relations one can find.

We first note, it is sufficient to consider only one specific $k$, because from (B5) one can derive straightforwardly the analogous relation for any other $k^{\prime}$. Second, it is sufficient to consider only the cases $j^{\prime} \leq j$, because $T_{j j^{\prime}}=T_{j^{\prime} j}^{*}$. The remaining relations certainly are independent because of the independency of the $t$-matrix elements. Choosing then first $j^{\prime}=j$, the case $j^{\prime}=k$ yields the identity, whereas for $j^{\prime} \neq k$ one finds

$$
T_{j^{\prime} j^{\prime}} T_{k k}=\left|T_{j^{\prime} k}\right|^{2},
$$

which constitute $(n-1)$ real quadratic relations

$$
\sum_{\alpha \alpha^{\prime}} \tau_{j^{\prime} j^{\prime}}^{\alpha} \tau_{k k}^{\alpha^{\prime}} \mathcal{O}^{\alpha} \mathcal{O}^{\alpha^{\prime}}=\sum_{\alpha \alpha^{\prime}} \tau_{j^{\prime} j}^{\alpha *} \tau_{j^{\prime} j}^{\alpha^{\prime}} \mathcal{O}^{\alpha} \mathcal{O}^{\alpha^{\prime}} .
$$

As next we consider the case $i<j$ for which one has $N=n(n-1) / 2$ different pairs. Again one can discard the cases $j^{\prime}=k$ or $j=k$, because they do not result in quadratic relations, thus ruling out $n-1$ relations. Therefore, one finds in this case $\left(j^{\prime}<j\right)$ a total number of

$$
N-(n-1)=\frac{1}{2}(n-1)(n-2)
$$

different complex quadratic relations of the form

$$
\sum_{\alpha \alpha^{\prime}} \tau_{j^{\prime} j}^{\alpha} \tau_{k k}^{\alpha^{\prime}} \mathcal{O}^{\alpha} \mathcal{O}^{\alpha^{\prime}}=\sum_{\alpha \alpha^{\prime}} \tau_{j^{\prime} k}^{\alpha} \tau_{k j}^{\alpha^{\prime}} \mathcal{O}^{\alpha} \mathcal{O}^{\alpha^{\prime}} .
$$

Separating these into real and imaginary parts, one finds as total number of independent real quadratic relations between observables

$$
(n-1)+2 \frac{1}{2}(n-1)(n-2)=(n-1)^{2},
$$

which is just the required number of relations in order to reduce the number of $n^{2}$ linearly independent observables to $n^{2}-(n-1)^{2}=2 n-1$ independent ones. 


\section{APPENDIX C: MULTIPOLE EXPANSION OF STRUCTURE FUNCTIONS AND FORM FACTORS}

Here we will list more explicit expressions for the multipole expansion of the structure functions of the differential cross section

$$
f_{a}^{(\prime) I M( \pm)}=\sum_{K} f_{a}^{(\prime) I M( \pm), K} d_{-M-\beta(a), 0}^{K}(\theta)
$$

As shown in detail in [10], one obtains for the coefficients of the structure functions

$$
\begin{aligned}
& f_{L}^{I M, K}=\frac{8 \sqrt{3}}{1+\delta_{M 0}} \pi \hat{I} \hat{K}^{2} \sum_{L^{\prime} \mu^{\prime} j^{\prime} L \mu j}(-)^{L} \sum_{J} \hat{J}^{2}\left(\begin{array}{ccc}
J & I & K \\
0 & M & -M
\end{array}\right)\left(\begin{array}{ccc}
L^{\prime} & L & J \\
0 & 0 & 0
\end{array}\right)\left\{\begin{array}{ccc}
j^{\prime} & j & K \\
L^{\prime} & L & J \\
1 & 1 & I
\end{array}\right\} \\
& \left((-)^{L^{\prime}+\mu^{\prime}+j^{\prime}}+1\right)\left((-)^{L+\mu+j}+1\right) \widetilde{\mathcal{D}}_{00}^{K 0}\left(\mu^{\prime} j^{\prime} \mu j\right) \Re e\left(i^{\delta_{I 1}} \widetilde{C}^{L^{\prime} *}\left(\mu^{\prime} j^{\prime}\right) \widetilde{C}^{l}(\mu j)\right), \\
& f_{T}^{I M, K}=-\frac{32 \sqrt{3}}{1+\delta_{M 0}} \pi \hat{I} \hat{K}^{2} \sum_{L^{\prime} \mu^{\prime} j^{\prime} L \mu j}(-)^{L} \sum_{J} \hat{J}^{2}\left(\begin{array}{ccc}
J & I & K \\
0 & M & -M
\end{array}\right)\left(\begin{array}{ccc}
L^{\prime} & L & J \\
1 & -1 & 0
\end{array}\right)\left\{\begin{array}{ccc}
j^{\prime} & j & K \\
L^{\prime} & L & J \\
1 & 1 & I
\end{array}\right\} \\
& \widetilde{\mathcal{D}}_{00}^{K 0}\left(\mu^{\prime} j^{\prime} \mu j\right) \Re e\left(i^{\delta_{I 1}} \widetilde{N}_{1}^{L^{\prime} *}\left(\mu^{\prime} j^{\prime}\right) \widetilde{N}_{1}^{L}(\mu j)\right), \\
& f_{L T}^{I M \pm, K}=\frac{16 \sqrt{6}}{1+\delta_{M 0}} \pi \hat{I} \hat{K}^{2} \sum_{L^{\prime} \mu^{\prime} j^{\prime} L \mu j}(-)^{L} \sum_{J} \hat{J}^{2}\left[\left(\begin{array}{ccc}
J & I & K \\
1 & M & -M-1
\end{array}\right) \pm(-)^{I+M}\left(\begin{array}{ccc}
J & I & K \\
1 & -M & M-1
\end{array}\right)\right] \\
& \left(\begin{array}{ccc}
L^{\prime} & L & J \\
0 & -1 & 1
\end{array}\right)\left\{\begin{array}{ccc}
j^{\prime} & j & K \\
L^{\prime} & L & J \\
1 & 1 & I
\end{array}\right\}\left((-)^{L^{\prime}+\mu^{\prime}+j^{\prime}}+1\right) \widetilde{\mathcal{D}}_{00}^{K 0}\left(\mu^{\prime} j^{\prime} \mu j\right) \Re e\left(i^{\delta_{I 1}} \widetilde{C}^{L^{\prime} *}\left(\mu^{\prime} j^{\prime}\right) \widetilde{N}_{1}^{L}(\mu j)\right) \\
& f_{T T}^{I M \pm, K}=-\frac{16 \sqrt{3}}{1+\delta_{M 0}} \pi \hat{I} \hat{K}^{2} \sum_{L^{\prime} \mu^{\prime} j^{\prime} L \mu j}(-)^{L} \sum_{J} \hat{J}^{2}\left[\left(\begin{array}{ccc}
J & I & K \\
2 & M & -M-2
\end{array}\right) \pm(-)^{I+M}\left(\begin{array}{ccc}
J & I & K \\
1 & -M & M-2
\end{array}\right)\right] \\
& \left(\begin{array}{ccc}
L^{\prime} & L & J \\
-1 & -1 & 2
\end{array}\right)\left\{\begin{array}{ccc}
j^{\prime} & j & K \\
L^{\prime} & L & J \\
1 & 1 & I
\end{array}\right\} \widetilde{\mathcal{D}}_{00}^{K 0}\left(\mu^{\prime} j^{\prime} \mu j\right) \Re e\left(i^{\delta_{I 1}} \widetilde{N}_{-1}^{L^{\prime} *}\left(\mu^{\prime} j^{\prime}\right) \widetilde{N}_{1}^{L}(\mu j)\right) \\
& f_{T}^{\prime I M, K}=\frac{32 \sqrt{3}}{1+\delta_{M 0}} \pi \hat{I} \hat{K}^{2} \sum_{L^{\prime} \mu^{\prime} j^{\prime} L \mu j}(-)^{L} \sum_{J} \hat{J}^{2}\left(\begin{array}{ccc}
J & I & K \\
0 & M & -M
\end{array}\right)\left(\begin{array}{ccc}
L^{\prime} & L & J \\
1 & -1 & 0
\end{array}\right)\left\{\begin{array}{ccc}
j^{\prime} & j & K \\
L^{\prime} & L & J \\
1 & 1 & I
\end{array}\right\} \\
& \widetilde{\mathcal{D}}_{00}^{K 0}\left(\mu^{\prime} j^{\prime} \mu j\right) \Im m\left(i^{\delta_{I 1}} \widetilde{N}_{1}^{L^{\prime} *}\left(\mu^{\prime} j^{\prime}\right) \widetilde{N}_{1}^{L}(\mu j)\right), \\
& f_{L T}^{\prime I M \pm, K}=-\frac{16 \sqrt{6}}{1+\delta_{M 0}} \pi \hat{I} \hat{K}^{2} \sum_{L^{\prime} \mu^{\prime} j^{\prime} L \mu j}(-)^{L} \sum_{J} \hat{J}^{2}\left[\left(\begin{array}{ccc}
J & I & K \\
1 & M & -M-1
\end{array}\right) \pm(-)^{I+M}\left(\begin{array}{ccc}
J & I & K \\
1 & -M & M-1
\end{array}\right)\right] \\
& \left(\begin{array}{ccc}
L^{\prime} & L & J \\
0 & -1 & 1
\end{array}\right)\left\{\begin{array}{ccc}
j^{\prime} & j & K \\
L^{\prime} & L & J \\
1 & 1 & I
\end{array}\right\}\left((-)^{L^{\prime}+\mu^{\prime}+j^{\prime}}+1\right) \widetilde{\mathcal{D}}_{00}^{K 0}\left(\mu^{\prime} j^{\prime} \mu j\right) \Im m\left(i^{\delta_{I 1}} \widetilde{C}^{L^{\prime} *}\left(\mu^{\prime} j^{\prime}\right) \widetilde{N}_{1}^{L}(\mu j)\right)
\end{aligned}
$$

Note, that the tilde indicates the incorporation of the hadronic phase factor $e^{i \delta_{\mu}^{j}}$ in the multipole matrix element. Now we specialize further in order to obtain the angular coefficients for the unpolarized differential cross section,

$$
S_{0}=c\left(k_{1}^{\mathrm{lab}}, k_{2}^{\mathrm{lab}}\right) \sum_{K}\left(\left[\rho_{L} f_{L}^{K}+\rho_{T} f_{T}^{K}\right] d_{00}^{K}(\theta)+\rho_{L T} f_{L T}^{K} d_{-10}^{K}(\theta) \cos \phi+\rho_{T T} f_{T T}^{K} d_{-20}^{K}(\theta) \cos 2 \phi\right),
$$


by setting $I=M=0$ in (C2) through (C7). Writing for simplicity $f_{L / T}^{K}$ and $f_{L T / T T}^{(\prime) K}$ instead of $f_{L / T}^{00, K}$ and $f_{L T / T T}^{(\prime) 00+, K}$, respectively, one obtains

$$
\begin{aligned}
& f_{L}^{K}=-4 \pi \hat{K}^{2} \sum_{L^{\prime} \mu^{\prime} j^{\prime} L \mu j}(-)^{L^{\prime}+L+j}\left(\begin{array}{ccc}
L^{\prime} & L & K \\
0 & 0 & 0
\end{array}\right)\left\{\begin{array}{ccc}
j^{\prime} & j & K \\
L & L^{\prime} & 1
\end{array}\right\} \\
& \left((-)^{L^{\prime}+\mu^{\prime}+j^{\prime}}+1\right)\left((-)^{L+\mu+j}+1\right) \widetilde{\mathcal{D}}_{00}^{K 0}\left(\mu^{\prime} j^{\prime} \mu j\right) \Re e\left(i^{\delta_{I 1}} \widetilde{C}^{L^{\prime} *}\left(\mu^{\prime} j^{\prime}\right) \widetilde{C}^{l}(\mu j)\right), \\
& f_{T}^{K}=16 \pi \hat{K}^{2} \sum_{L^{\prime} \mu^{\prime} j^{\prime} L \mu j}(-)^{L^{\prime}+L+j}\left(\begin{array}{ccc}
L^{\prime} & L & K \\
1 & -1 & 0
\end{array}\right)\left\{\begin{array}{ccc}
j^{\prime} & j & K \\
L & L^{\prime} & 1
\end{array}\right\} \\
& \widetilde{\mathcal{D}}_{00}^{K 0}\left(\mu^{\prime} j^{\prime} \mu j\right) \Re e\left(i^{\delta_{I 1}} \widetilde{N}_{1}^{L^{\prime} *}\left(\mu^{\prime} j^{\prime}\right) \widetilde{N}_{1}^{L}(\mu j)\right), \\
& f_{L T}^{K}=16 \sqrt{2} \pi \hat{K}^{2} \sum_{L^{\prime} \mu^{\prime} j^{\prime} L \mu j}(-)^{L^{\prime}+L+j}\left(\begin{array}{ccc}
L^{\prime} & L & K \\
0 & -1 & 1
\end{array}\right)\left\{\begin{array}{ccc}
j^{\prime} & j & K \\
L & L^{\prime} & 1
\end{array}\right\} \\
& \left((-)^{L^{\prime}+\mu^{\prime}+j^{\prime}}+1\right) \widetilde{\mathcal{D}}_{00}^{K 0}\left(\mu^{\prime} j^{\prime} \mu j\right) \Re e\left(i^{\delta_{I 1}} \widetilde{C}^{L^{\prime} *}\left(\mu^{\prime} j^{\prime}\right) \widetilde{N}_{1}^{L}(\mu j)\right), \\
& f_{T T}^{K}=16 \pi \hat{K}^{2} \sum_{L^{\prime} \mu^{\prime} j^{\prime} L \mu j}(-)^{L^{\prime}+L+j}\left(\begin{array}{ccc}
L^{\prime} & L & K \\
-1 & -1 & 2
\end{array}\right)\left\{\begin{array}{ccc}
j^{\prime} & j & K \\
L & L^{\prime} & 1
\end{array}\right\} \\
& \widetilde{\mathcal{D}}_{00}^{K 0}\left(\mu^{\prime} j^{\prime} \mu j\right) \Re e\left(i^{\delta_{I 1}} \widetilde{N}_{-1}^{L^{\prime} *}\left(\mu^{\prime} j^{\prime}\right) \widetilde{N}_{1}^{L}(\mu j)\right) .
\end{aligned}
$$

At the end of this appendix, we will give the explicit multipole decomposition of the various inclusive form factors of $d\left(e, e^{\prime}\right) n p$, which can be obtained from the $(K=0)$-coefficients of $(\mathrm{C} 2)-(\mathrm{C} 7)$ according to

$$
F_{a}^{(\prime) I-M}=(-)^{I+M}\left(1+\delta_{M 0}\right) \frac{\pi}{3}\left(f_{a}^{(\prime) I M+, 0}-f_{a}^{(\prime) I M-, 0}\right) .
$$

The unpolarized form factors are given by

$$
\begin{aligned}
& F_{L}=\frac{16 \pi^{2}}{3} \sum_{L j \mu} \frac{e^{-2 \rho_{\mu}^{j}}}{2 L+1}\left|C^{L}(\mu j)\right|^{2}, \\
& F_{T}=\frac{16 \pi^{2}}{3} \sum_{L j \mu} \frac{e^{-2 \rho_{\mu}^{j}}}{2 L+1}\left(\left|E^{L}(\mu j)\right|^{2}+\left|M^{L}(\mu j)\right|^{2}\right),
\end{aligned}
$$

the vector polarization form factors by

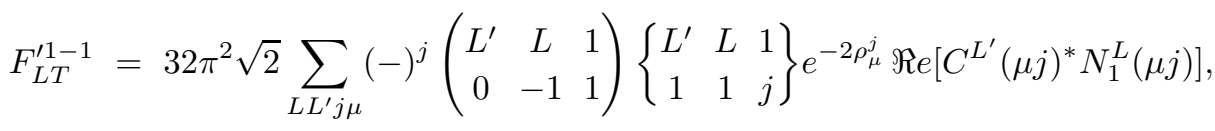

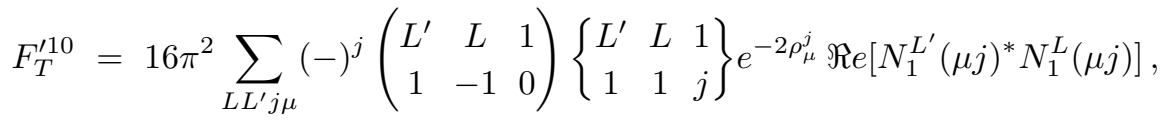

and finally the tensor polarization form factors by

$$
\begin{aligned}
& F_{L}^{20}=-16 \pi^{2} \sqrt{\frac{5}{3}} \sum_{L L^{\prime} j \mu}(-)^{j}\left(\begin{array}{ccc}
L^{\prime} & L & 2 \\
0 & 0 & 0
\end{array}\right)\left\{\begin{array}{ccc}
L^{\prime} & L & 2 \\
1 & 1 & j
\end{array}\right\} e^{-2 \rho_{\mu}^{j}} \Re e\left[C^{L^{\prime}}(\mu j)^{*} C^{L}(\mu j)\right],
\end{aligned}
$$

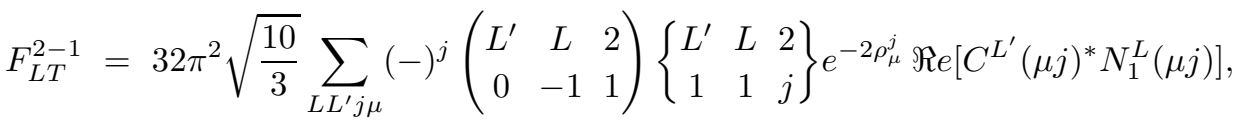

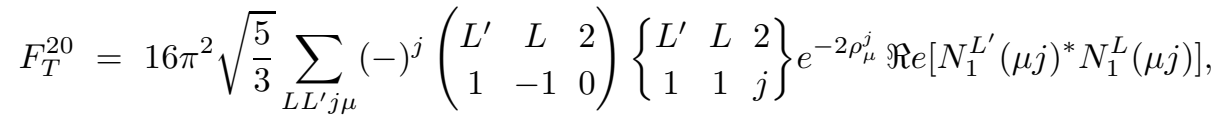

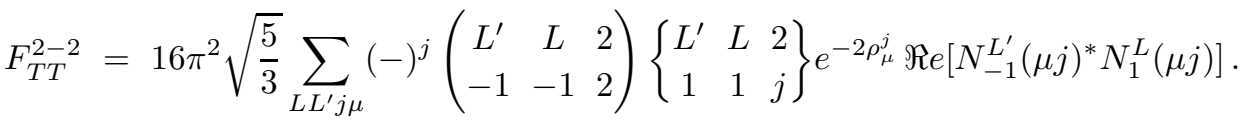


These expressions have already been reported before in [1]. The following two additional form factors vanish below pion threshold if time reversal invariance holds. However, if one considers the partial inclusive reaction $d\left(e, e^{\prime}\right) n p$, they become nonvanishing above pion threshold in a coupled channel approach including isobars without explicit treatment of the $N N \pi$-channels [1].

$$
\begin{aligned}
& F_{L T}^{1-1}=-32 \pi^{2} \sqrt{2} \sum_{L L^{\prime} j \mu}(-)^{j}\left(\begin{array}{ccc}
L^{\prime} & L & 1 \\
0 & -1 & 1
\end{array}\right)\left\{\begin{array}{ccc}
L^{\prime} & L & 1 \\
1 & 1 & j
\end{array}\right\} e^{-2 \rho_{\mu}^{j} \Im m\left[C^{L^{\prime}}(\mu j)^{*} N_{1}^{L}(\mu j)\right],} \\
& F_{L T}^{\prime 2-1}=-32 \pi^{2} \sqrt{\frac{10}{3}} \sum_{L L^{\prime} j \mu}(-)^{j}\left(\begin{array}{ccc}
L^{\prime} & L & 2 \\
0 & -1 & 1
\end{array}\right)\left\{\begin{array}{ccc}
L^{\prime} & L & 2 \\
1 & 1 & j
\end{array}\right\} e^{-2 \rho_{\mu}^{j}} \Im m\left[C^{L^{\prime}}(\mu j)^{*} N_{1}^{L}(\mu j)\right] .
\end{aligned}
$$

We would like to emphasize, that if one considers the completely inclusive process $d\left(e, e^{\prime}\right) X$, the corresponding additional form factors will vanish as long as time reversal invariance holds.

\section{APPENDIX D: SEPARATION OF A SPECIFIC STRUCTURE FUNCTION}

The first step in the determination of any structure function is the measurement of that asymmetry to which it contributes. This requires already a number of measurements with different electron and deuteron polarization parameters, i.e., two for $A_{0}(X), A_{e}(X), A_{d}^{V}(X)$, and $A_{d}^{T}(X)$ and four for $A_{e d}^{V}(X)$ and $A_{e d}^{T}(X)$. Next one must determine how many different settings of the angles $\phi, \phi_{d}$ and $\theta_{d}$ are necessary for the final separation. As one will see in the following, the structure functions can be divided into different classes according to the minimum number of asymmetry measurements required for their extraction. A quick glance at (83) shows that the six asymmetries contain differing numbers of structure functions, taking for example an observable of type $A$, one finds four in $A_{0}(X)$, one in $A_{e}(X), 8$ in $A_{d}^{V}(X), 16$ in $A_{d}^{T}(X), 5$ in $A_{e d}^{V}(X)$, and 7 in $A_{e d}^{T}(X)$. Similar numbers are found for an observable of type $B$.

First we will consider an observable of type $A$. Obviously, the simplest case is the electron asymmetry $A_{e}(X)$ containing only $f_{L T}^{\prime}(X)$. This means that $f_{L T}^{\prime}(X)$ can be determined from just one asymmetry measurement. Similarly a close inspection of the dependence of the asymmetries on the angles $\phi, \phi_{d}$ and $\theta_{d}$ shows that four other structure functions and two combinations need only one asymmetry measurement. These are, introducing for convenience

$$
S_{d / e d}^{V / T}\left(X ; \phi, \tilde{\phi}, \theta_{d}\right)=S_{0}(\phi) A_{d / e d}^{V / T}\left(X ; \phi, \tilde{\phi}, \theta_{d}\right)
$$

in order to exhibit the angular dependence explicitly,

$$
\begin{aligned}
c\left(k_{1}^{\mathrm{lab}}, k_{2}^{\mathrm{lab}}\right) \rho_{L T} f_{L T}^{10}(X) & =S_{d}^{V}\left(X ; \frac{\pi}{2}, \tilde{\phi}, 0\right), \\
c\left(k_{1}^{\mathrm{lab}}, k_{2}^{\mathrm{lab}}\right) \rho_{L T} f_{L T}^{11,-}(X) & =-\sqrt{2} S_{d}^{V}\left(X ; \frac{\pi}{2}, 0, \frac{\pi}{2}\right), \\
c\left(k_{1}^{\mathrm{lab}}, k_{2}^{\mathrm{lab}}\right) \rho_{T}^{\prime} f_{T}^{\prime 10}(X) & =-S_{e d}^{V}\left(X ; \frac{\pi}{2}, 0,0\right), \\
c\left(k_{1}^{\mathrm{lab}}, k_{2}^{\mathrm{lab}}\right) \rho_{T}^{\prime} f_{T}^{\prime 11}(X) & =\sqrt{2} S_{e d}^{V} X ;\left(\frac{\pi}{2}, 0, \frac{\pi}{2}\right), \\
c\left(k_{1}^{\mathrm{lab}}, k_{2}^{\mathrm{lab}}\right) \rho_{L T}^{\prime} f_{L T}^{\prime 11,+}(X) & -\sqrt{2} S_{e d}^{V}\left(X ; \frac{\pi}{2}, \frac{\pi}{2}, \frac{\pi}{2}\right), \\
c\left(k_{1}^{\mathrm{lab}}, k_{2}^{\mathrm{lab}}\right) \rho_{L T}^{\prime} f_{L T}^{\prime 20}(X) & =S_{e d}^{T}\left(X ; \frac{\pi}{2}, \tilde{\phi}, 0\right) .
\end{aligned}
$$

Nine other structure functions and seven combinations require only two measurements for their separation. These 
are

$$
\begin{aligned}
& c\left(k_{1}^{\mathrm{lab}}, k_{2}^{\mathrm{lab}}\right) \rho_{T T} f_{T T}^{10}(X)=\frac{2}{\sqrt{3}} S_{d}^{V}\left(X ; \frac{\pi}{3}, \tilde{\phi}, 0\right)-S_{d}^{V}\left(X ; \frac{\pi}{2}, \tilde{\phi}, 0\right), \\
& c\left(k_{1}^{\mathrm{lab}}, k_{2}^{\mathrm{lab}}\right) \rho_{L T} f_{L T}^{11,+}(X)=-\left[S_{d}^{V}\left(X ; \frac{\pi}{4}, \frac{\pi}{2}, \frac{\pi}{2}\right)-S_{d}^{V}\left(X ; \frac{3 \pi}{4}, \frac{\pi}{2}, \frac{\pi}{2}\right)\right], \\
& c\left(k_{1}^{\mathrm{lab}}, k_{2}^{\mathrm{lab}}\right) \rho_{T T} f_{T T}^{11,-}(X)=\sqrt{\frac{2}{3}}\left[2 S_{d}^{V}\left(X ; \frac{\pi}{3}, 0, \frac{\pi}{2}\right)-\sqrt{3} S_{d}^{V}\left(X ; \frac{\pi}{2}, 0, \frac{\pi}{2}\right],\right. \\
& c\left(k_{1}^{\mathrm{lab}}, k_{2}^{\mathrm{lab}}\right) \rho_{L T} f_{L T}^{20}(X)=\frac{1}{\sqrt{2}}\left[S_{d}^{T}\left(X ; \frac{\pi}{4}, \tilde{\phi}, 0\right)-S_{d}^{T}\left(X ; \frac{3 \pi}{4}, \tilde{\phi}, 0\right)\right], \\
& c\left(k_{1}^{\mathrm{lab}}, k_{2}^{\mathrm{lab}}\right) \rho_{L T} f_{L T}^{21+}(X)=\sqrt{\frac{3}{2}}\left[S_{d}^{T}\left(X ; \pi, \frac{\pi}{4}, \theta_{d}^{0}\right)-S_{d}^{T}\left(X ; 0, \frac{\pi}{4}, \theta_{d}^{0}\right)\right], \\
& c\left(k_{1}^{\mathrm{lab}}, k_{2}^{\mathrm{lab}}\right) \rho_{L T} f_{L T}^{21,-}(X)=\frac{\sqrt{3}}{2}\left[S_{d}^{T}\left(X ; \frac{\pi}{2}, \frac{\pi}{2}, \theta_{d}^{0}\right)-S_{d}^{T}\left(X ; \frac{\pi}{2},-\frac{\pi}{2}, \theta_{d}^{0}\right)\right], \\
& c\left(k_{1}^{\mathrm{lab}}, k_{2}^{\mathrm{lab}}\right) \rho_{L T} f_{L T}^{22+}(X)=\sqrt{\frac{3}{2}}\left[S_{d}^{T}\left(X ; \pi, \frac{\pi}{2}, \theta_{d}^{0}\right)-S_{d}^{T}\left(X ; 0, \frac{\pi}{2}, \theta_{d}^{0}\right)\right], \\
& c\left(k_{1}^{\mathrm{lab}}, k_{2}^{\mathrm{lab}}\right) \rho_{L T} f_{L T}^{22,-}(X)=-\sqrt{\frac{2}{3}}\left[2 S_{d}^{T}\left(X ; \frac{\pi}{2}, \frac{\pi}{4}, \frac{\pi}{2}\right)+S_{d}^{T}\left(X ; \frac{3 \pi}{2}, \frac{\pi}{4}, 0\right)\right], \\
& c\left(k_{1}^{\mathrm{lab}}, k_{2}^{\mathrm{lab}}\right) \rho_{L T}^{\prime} f_{L T}^{\prime 10}(X)=-\left[S_{e d}^{V}(X ; 0, \tilde{\phi}, 0)-S_{e d}^{V}\left(X ; \frac{\pi}{2}, \tilde{\phi}, 0\right)\right], \\
& c\left(k_{1}^{\mathrm{lab}}, k_{2}^{\mathrm{lab}}\right) \rho_{L T}^{\prime} f_{L T}^{\prime 11,-}(X)=\sqrt{2}\left[S_{e d}^{V}\left(X ; 0,0, \frac{\pi}{2}\right)-S_{e d}^{V}\left(X ; \frac{\pi}{2}, 0, \frac{\pi}{2}\right)\right], \\
& c\left(k_{1}^{\mathrm{lab}}, k_{2}^{\mathrm{lab}}\right) \rho_{T}^{\prime} f_{T}^{\prime 21}(X)=-\frac{\sqrt{3}}{2}\left[S_{e d}^{T}\left(X ; 0, \frac{\pi}{2}, \theta_{d}^{0}\right)+S_{e d}^{T}\left(X ; \pi, \frac{\pi}{2}, \theta_{d}^{0}\right)\right], \\
& c\left(k_{1}^{\mathrm{lab}}, k_{2}^{\mathrm{lab}}\right) \rho_{T}^{\prime} f_{T}^{\prime 22}(X)=\sqrt{\frac{2}{3}}\left[2 S_{e d}^{T}\left(X ; \frac{\pi}{2}, \frac{\pi}{4}, \frac{\pi}{2}\right)+S_{e d}^{T}\left(X ; \frac{\pi}{2}, \frac{\pi}{4}, 0\right)\right], \\
& c\left(k_{1}^{\mathrm{lab}}, k_{2}^{\mathrm{lab}}\right) \rho_{L T}^{\prime} f_{L T}^{\prime 21,-}(X)=-\frac{\sqrt{3}}{2}\left[S_{e d}^{T}\left(X ; 0, \frac{\pi}{2}, \theta_{d}^{0}\right)-S_{e d}^{T}\left(X ; \pi, \frac{\pi}{2}, \theta_{d}^{0}\right)\right], \\
& c\left(k_{1}^{\mathrm{lab}}, k_{2}^{\mathrm{lab}}\right) \rho_{L T}^{\prime} f_{L T}^{\prime 21,+}(X)=-\frac{\sqrt{3}}{2}\left[S_{e d}^{T}\left(X ; \frac{\pi}{2}, 0, \theta_{d}^{0}\right)-S_{e d}^{T}\left(X ; \frac{\pi}{2}, \pi, \theta_{d}^{0}\right)\right], \\
& c\left(k_{1}^{\mathrm{lab}}, k_{2}^{\mathrm{lab}}\right) \rho_{L T}^{\prime} f_{L T}^{\prime 22,+}(X)=\sqrt{\frac{3}{2}}\left[S_{e d}^{T}\left(X ; \frac{\pi}{2}, 0, \theta_{d}^{0}\right)+S_{e d}^{T}\left(X ; \frac{\pi}{2}, \pi, \theta_{d}^{0}\right)\right] \\
& \stackrel{\text { or }}{=} \sqrt{\frac{2}{3}}\left[2 S_{e d}^{T}\left(X ; \frac{\pi}{2}, 0, \frac{\pi}{2}\right)+S_{e d}^{T}\left(X ; \frac{\pi}{2}, 0,0\right)\right], \\
& c\left(k_{1}^{\mathrm{lab}}, k_{2}^{\mathrm{lab}}\right) \rho_{L T}^{\prime} f_{L T}^{\prime 22-}(X)=\sqrt{\frac{2}{3}}\left[S_{e d}^{T}\left(X ; 0, \frac{\pi}{4}, \frac{\pi}{2}\right)-S_{e d}^{T}\left(X ; \pi, \frac{\pi}{4}, \frac{\pi}{2}\right)\right],
\end{aligned}
$$

where $\theta_{d}^{0}=\operatorname{arcos}(1 / \sqrt{3})$ (see Sect. III).

The terms $\rho_{L} f_{L}^{11}+\rho_{T} f_{T}^{11}$ in $A_{d}^{V}$ and $\rho_{L} f_{L}^{20}+\rho_{T} f_{T}^{20}$ in $A_{d}^{T}$ can also be determined from two asymmetry measurements, i.e.,

$$
\begin{aligned}
& c\left(k_{1}^{\mathrm{lab}}, k_{2}^{\mathrm{lab}}\right)\left(\rho_{L} f_{L}^{11}+\rho_{T} f_{T}^{11}(X)\right)=-\frac{1}{\sqrt{2}}\left[S_{d}^{V}\left(X ; \frac{\pi}{4}, \frac{\pi}{2}, \frac{\pi}{2}\right)+S_{d}^{V}\left(X ; \frac{3 \pi}{4}, \frac{\pi}{2}, \frac{\pi}{2}\right)\right], \\
& c\left(k_{1}^{\mathrm{lab}}, k_{2}^{\mathrm{lab}}\right)\left(\rho_{L} f_{L}^{20}+\rho_{T} f_{T}^{20}(X)\right)=\frac{1}{2}\left[S_{d}^{T}\left(X ; \frac{\pi}{4}, \tilde{\phi}, 0\right)+S_{d}^{T}\left(X ; \frac{3 \pi}{4}, \tilde{\phi}, 0\right)\right] .
\end{aligned}
$$

In order to separate the longitudinal from the transverse part one needs in addition a RosenBLUTH analysis.

Increasing the number of asymmetry measurements to three allows one to determine only one further structure function and one combination, namely

$$
\begin{aligned}
c\left(k_{1}^{\mathrm{lab}}, k_{2}^{\mathrm{lab}}\right) \rho_{T T} f_{T T}^{11,+}(X) & =-\frac{1}{\sqrt{2}}\left[S_{d}^{V}\left(X ; \frac{\pi}{4}, \frac{\pi}{2}, \frac{\pi}{2}\right)+S_{d}^{V}\left(X ; \frac{3 \pi}{4}, \frac{\pi}{2}, \frac{\pi}{2}\right)-2 S_{d}^{V}\left(X ; \frac{\pi}{2}, \frac{\pi}{2}, \frac{\pi}{2}\right)\right], \\
c\left(k_{1}^{\mathrm{lab}}, k_{2}^{\mathrm{lab}}\right) \rho_{T T} f_{T T}^{20}(X) & =\frac{1}{2}\left[S_{d}^{T}\left(X ; \frac{\pi}{4}, \tilde{\phi}, 0\right)+S_{d}^{T}\left(X ; \frac{3 \pi}{4}, \tilde{\phi}, 0\right)\right]-S_{d}^{T}\left(X ; \frac{3 \pi}{2}, \tilde{\phi}, 0\right) .
\end{aligned}
$$


All thirteen vector structure functions are then determined and fifteen of the tensor ones or respective combinations. For the remaining combinations $f_{T T}^{21,+}(X), f_{T T}^{21,-}(X), f_{T T}^{22,+}(X), f_{T T}^{22,-}(X),\left(\rho_{L} f_{L}^{21}+\rho_{T} f_{T}^{21}(X)\right)$, and $\left(\rho_{L} f_{L}^{22}+\rho_{T} f_{T}^{22}(X)\right)$ one needs four measurements in order to determine them. The last two combinations require a RosEnbluth separation in addition.

Now we turn to the separation of structure functions for $B$-type. Without target and electron polarization one has two structure functions, one of which can be determined by one out-of-plane measurements, namely $\left(f_{L T}^{00-}(X), \phi=\frac{\pi}{2}\right)$, and the other needs two, $\left(f_{T T}^{00-}(X), \phi=\frac{\pi}{4}, \frac{\pi}{2}\right)$. Electron polarization alone without deuteron polarization leads to two other structure functions: $f_{T}^{\prime 00-}(X)$ which can be obtained from one out-of-plane measurement at $\phi=\frac{\pi}{2}$, and $f_{L T}^{\prime 00}(X)$, which requires two settings.

For the structure functions with target polarization we find that six of them can be determined by a single asymmetry measurement. They are

$$
\begin{aligned}
c\left(k_{1}^{\mathrm{lab}}, k_{2}^{\mathrm{lab}}\right) \rho_{L T} f_{L T}^{11-}(X) & =\sqrt{2} S_{d}^{V}\left(X ; \frac{\pi}{2}, \frac{\pi}{2}, \frac{\pi}{2}\right), \\
c\left(k_{1}^{\mathrm{lab}}, k_{2}^{\mathrm{lab}}\right) \rho_{L T} f_{L T}^{20-}(X) & =S_{d}^{T}\left(X ; \frac{\pi}{2}, \tilde{\phi}, 0\right), \\
c\left(k_{1}^{\mathrm{lab}}, k_{2}^{\mathrm{lab}}\right) \rho_{T}^{\prime} f_{T}^{\prime 11}(X) & =-\sqrt{2} S_{e d}^{V}\left(X ; \frac{\pi}{2}, \frac{\pi}{2}, \frac{\pi}{2}\right), \\
c\left(k_{1}^{\mathrm{lab}}, k_{2}^{\mathrm{lab}}\right) \rho_{T}^{\prime} f_{T}^{\prime 20}(X) & =-S_{e d}^{T}\left(X ; \frac{\pi}{2}, \tilde{\phi}, 0\right), \\
c\left(k_{1}^{\mathrm{lab}}, k_{2}^{\mathrm{lab}}\right) \rho_{L T}^{\prime} f_{L T}^{\prime 10+}(X) & =S_{e d}^{V}\left(X ; \frac{\pi}{2}, \tilde{\phi}, 0\right), \\
c\left(k_{1}^{\mathrm{lab}}, k_{2}^{\mathrm{lab}}\right) \rho_{L T}^{\prime} f_{L T}^{\prime 11+}(X) & =-\sqrt{2} S_{e d}^{V}\left(X ; \frac{\pi}{2}, 0, \frac{\pi}{2}\right) .
\end{aligned}
$$


Sixteen other structure functions require only two measurements for their separation. These are

$$
\begin{aligned}
& c\left(k_{1}^{\mathrm{lab}}, k_{2}^{\mathrm{lab}}\right) \rho_{L T} f_{L T}^{10+}(X)=\frac{1}{\sqrt{2}}\left[S_{d}^{V}\left(X ; \frac{\pi}{4}, \tilde{\phi}, 0\right)-S_{d}^{V}\left(X ; \frac{3 \pi}{4}, \tilde{\phi}, 0\right)\right], \\
& c\left(k_{1}^{\mathrm{lab}}, k_{2}^{\mathrm{lab}}\right) \rho_{L T} f_{L T}^{11+}(X)=-\left[S_{d}^{V}\left(X ; \frac{\pi}{4}, 0, \frac{\pi}{2}\right)-S_{d}^{V}\left(X ; \frac{3 \pi}{4}, 0, \frac{\pi}{2}\right)\right], \\
& c\left(k_{1}^{\mathrm{lab}}, k_{2}^{\mathrm{lab}}\right) \rho_{L T} f_{L T}^{21+}(X)=\sqrt{\frac{2}{3}}\left[S_{d}^{T}\left(X ; \pi, \frac{\pi}{2}, \frac{\pi}{4}\right)-S_{d}^{T}\left(X ; 0, \frac{\pi}{2}, \frac{\pi}{4}\right)\right], \\
& c\left(k_{1}^{\mathrm{lab}}, k_{2}^{\mathrm{lab}}\right) \rho_{L T} f_{L T}^{21-}(X)=-\sqrt{\frac{2}{3}}\left[S_{d}^{T}\left(X ; \frac{\pi}{2}, 0, \frac{\pi}{4}\right)-S_{d}^{T}\left(X ; \frac{\pi}{2}, 0, \frac{3 \pi}{4}\right)\right], \\
& c\left(k_{1}^{\mathrm{lab}}, k_{2}^{\mathrm{lab}}\right) \rho_{L T} f_{L T}^{22+}(X)=\sqrt{\frac{2}{3}}\left[S_{d}^{T}\left(X ; 0, \frac{\pi}{4}, \frac{\pi}{2}\right)-S_{d}^{T}\left(X ; \pi, \frac{\pi}{4}, \frac{\pi}{2}\right)\right], \\
& c\left(k_{1}^{\mathrm{lab}}, k_{2}^{\mathrm{lab}}\right) \rho_{L T} f_{L T}^{22-}(X)=2 \sqrt{\frac{2}{3}}\left[S_{d}^{T}\left(X ; \frac{\pi}{2}, 0, \frac{\pi}{2}\right)+\frac{1}{2} S_{d}^{T}\left(X ; \frac{\pi}{2}, \tilde{\phi}, 0\right)\right], \\
& c\left(k_{1}^{\mathrm{lab}}, k_{2}^{\mathrm{lab}}\right) \rho_{T T} f_{T T}^{11-}(X)=-S_{d}^{V}\left(X ; \frac{\pi}{2}, \frac{\pi}{2}, \frac{\pi}{2}\right)+\sqrt{2} S_{d}^{V}\left(X ; \frac{\pi}{4}, \frac{\pi}{2}, \frac{\pi}{2}\right), \\
& c\left(k_{1}^{\mathrm{lab}}, k_{2}^{\mathrm{lab}}\right) \rho_{T T} f_{T T}^{20-}(X)=S_{d}^{T}\left(X ; \frac{\pi}{4}, \tilde{\phi}, 0\right)-\frac{1}{\sqrt{2}} S_{d}^{T}\left(X ; \frac{\pi}{2}, \tilde{\phi}, 0\right), \\
& c\left(k_{1}^{\mathrm{lab}}, k_{2}^{\mathrm{lab}}\right) \rho_{T}^{\prime} f_{T}^{\prime 21}(X)=\sqrt{\frac{3}{2}}\left[S_{e d}^{T}\left(X ; 0, \frac{\pi}{4}, \theta_{d}^{0}\right)+S_{e d}^{T}\left(X ; \pi, \frac{\pi}{4}, \theta_{d}^{0}\right)\right], \\
& c\left(k_{1}^{\mathrm{lab}}, k_{2}^{\mathrm{lab}}\right) \rho_{T}^{\prime} f_{T}^{\prime 22}(X)=\sqrt{\frac{3}{2}}\left[S_{e d}^{T}\left(X ; 0, \frac{\pi}{2}, \theta_{d}^{0}\right)+S_{e d}^{T}\left(X ; \pi, \frac{\pi}{2}, \theta_{d}^{0}\right)\right], \\
& c\left(k_{1}^{\mathrm{lab}}, k_{2}^{\mathrm{lab}}\right) \rho_{L T}^{\prime} f_{L T}^{\prime 11-}(X)=-\sqrt{2}\left[S_{e d}^{V}\left(X ; 0, \frac{\pi}{2}, \frac{\pi}{2}\right)-S_{e d}^{V}\left(X ; \frac{\pi}{2}, \frac{\pi}{2}, \frac{\pi}{2}\right)\right], \\
& c\left(k_{1}^{\mathrm{lab}}, k_{2}^{\mathrm{lab}}\right) \rho_{L T}^{\prime} f_{L T}^{\prime 20-}(X)=S_{e d}^{T}\left(X ; \frac{\pi}{2}, \tilde{\phi}, 0\right)-S_{e d}^{T}(X ; 0, \tilde{\phi}, 0), \\
& c\left(k_{1}^{\mathrm{lab}}, k_{2}^{\mathrm{lab}}\right) \rho_{L T}^{\prime} f_{L T}^{\prime 21+}(X)=-\sqrt{\frac{2}{3}}\left[S_{e d}^{T}\left(X ; \frac{\pi}{2}, \frac{\pi}{2}, \frac{\pi}{4}\right)-S_{e d}^{T}\left(X ; \frac{\pi}{2}, \frac{\pi}{2}, \frac{3 \pi}{4}\right)\right], \\
& c\left(k_{1}^{\mathrm{lab}}, k_{2}^{\mathrm{lab}}\right) \rho_{L T}^{\prime} f_{L T}^{\prime 21-}(X)=\sqrt{\frac{3}{2}}\left[S_{e d}^{T}\left(X ; 0, \frac{\pi}{4}, \theta_{d}^{0}\right)-S_{e d}^{T}\left(X ; \pi, \frac{\pi}{4}, \theta_{d}^{0}\right)\right], \\
& c\left(k_{1}^{\mathrm{lab}}, k_{2}^{\mathrm{lab}}\right) \rho_{L T}^{\prime} f_{L T}^{\prime 22+}(X)=\sqrt{\frac{2}{3}}\left[2 S_{e d}^{T}\left(X ; \frac{\pi}{2}, \frac{\pi}{4}, \frac{\pi}{2}\right)+S_{e d}^{T}\left(X ; \frac{\pi}{2}, \tilde{\phi}, 0\right)\right], \\
& c\left(k_{1}^{\mathrm{lab}}, k_{2}^{\mathrm{lab}}\right) \rho_{L T}^{\prime} f_{L T}^{\prime 22-}(X)=\sqrt{\frac{3}{2}}\left[S_{e d}^{T}\left(X ; 0, \frac{\pi}{2}, \theta_{d}^{0}\right)-S_{e d}^{T}\left(X ; \pi, \frac{\pi}{2}, \theta_{d}^{0}\right)\right] .
\end{aligned}
$$

In this last set of equations as well as for what follows we have made a particular choice of angles to allow the separations. This choice is not unique and other choices will lead to different coefficients in the linear combinations. The terms $\rho_{L} f_{L}^{10}(X)+\rho_{T} f_{T}^{10}(X)$ and $\rho_{L} f_{L}^{11}(X)+\rho_{T} f_{T}^{11}(X)$ in $A_{d}^{V}(X)$ can also be determined from two asymmetry measurements, i.e.,

$$
\begin{aligned}
c\left(k_{1}^{\mathrm{lab}}, k_{2}^{\mathrm{lab}}\right) \rho_{L} f_{L}^{10}(X)+\rho_{T} f_{T}^{10}(X) & =\frac{1}{2}\left[S_{d}^{V}\left(X ; \frac{\pi}{4}, \tilde{\phi}, 0\right)+S_{d}^{V}\left(X ; \frac{3 \pi}{4}, \tilde{\phi}, 0\right)\right] \\
c\left(k_{1}^{\mathrm{lab}}, k_{2}^{\mathrm{lab}}\right) \rho_{L} f_{L}^{11}(X)+\rho_{T} f_{T}^{11}(X) & =-\frac{1}{\sqrt{2}}\left[S_{d}^{V}\left(X ; \frac{\pi}{4}, 0, \frac{\pi}{2}\right)+S_{d}^{V}\left(X ; \frac{3 \pi}{4}, 0, \frac{\pi}{2}\right)\right] .
\end{aligned}
$$

In order to separate the longitudinal from the transverse part one needs in addition a RosENBLUTH analysis.

Increasing the number of asymmetry measurements to three allows one to determine only two further structure functions, namely

$$
\begin{aligned}
& c\left(k_{1}^{\mathrm{lab}}, k_{2}^{\mathrm{lab}}\right) \rho_{T T} f_{T T}^{11+}(X)=-\frac{1}{\sqrt{2}}\left[S_{d}^{V}\left(X ; \frac{\pi}{4}, 0, \frac{\pi}{2}\right)+S_{d}^{V}\left(X ; \frac{3 \pi}{4}, 0, \frac{\pi}{2}\right)-2 S_{d}^{V}\left(X ; \frac{\pi}{2}, 0, \frac{\pi}{2}\right)\right] \\
& c\left(k_{1}^{\mathrm{lab}}, k_{2}^{\mathrm{lab}}\right) \rho_{T T} f_{T T}^{10+}(X)=\frac{1}{2}\left[S_{d}^{V}\left(X ; \frac{\pi}{4}, \tilde{\phi}, 0\right)+S_{d}^{V}\left(X ; \frac{3 \pi}{4}, \tilde{\phi}, 0\right)\right]-S_{d}^{V}\left(X ; \frac{\pi}{2}, \tilde{\phi}, 0\right) .
\end{aligned}
$$


TABLE X: Number of asymmetry measurements for a structure function of an observable $X$. The symbol $(R)$ indicates the need of an additional Rosenbluth $L$ - $T$-separation.

\begin{tabular}{ccccccccc}
\hline \hline \multicolumn{7}{c}{$X \in A$} \\
\hline$I M$ & $L, T$ & $L T+$ & $L T-$ & $T T+$ & $T T-$ & $T^{\prime}$ & $L T^{\prime}+$ & $L T^{\prime}-$ \\
\hline 10 & - & - & 1 & - & 2 & 1 & - & 2 \\
11 & $2(\mathrm{R})$ & 2 & 1 & 3 & 2 & 1 & 1 & 2 \\
20 & $2(\mathrm{R})$ & 2 & - & 3 & - & - & 1 & - \\
21 & $4(\mathrm{R})$ & 2 & 2 & 4 & 4 & 2 & 2 & 2 \\
22 & $4(\mathrm{R})$ & 2 & 2 & 4 & 4 & 2 & 2 & 2 \\
\hline & & & & $X \in B$ & & & & \\
\hline$I M$ & $L, T$ & $L T+$ & $L T-$ & $T T+$ & $T T-$ & $T^{\prime}$ & $L T^{\prime}+$ & \\
\hline 10 & $2(\mathrm{R})$ & 2 & - & 3 & - & - & 1 & 2 \\
11 & $2(\mathrm{R})$ & 2 & 1 & 3 & 2 & 1 & 1 & 2 \\
20 & - & - & 1 & - & 2 & 1 & - & 2 \\
21 & $4(\mathrm{R})$ & 2 & 2 & 4 & 4 & 2 & 2 & 2 \\
22 & $3(\mathrm{R})$ & 2 & 2 & 4 & 4 & 2 & 2 & 2 \\
\hline \hline
\end{tabular}

Three settings also allow a determination of the combination

$$
c\left(k_{1}^{\mathrm{lab}}, k_{2}^{\mathrm{lab}}\right) \rho_{L} f_{L}^{22}(X)+\rho_{T} f_{T}^{22}(X)=\sqrt{\frac{2}{3}}\left[S_{d}^{T}\left(X ; \frac{\pi}{4}, \frac{\pi}{4}, \frac{\pi}{2}\right)+S_{d}^{T}\left(X ; \frac{3 \pi}{4}, \frac{\pi}{4}, \frac{\pi}{2}\right)+\frac{1}{\sqrt{2}} S_{d}^{T}\left(X ; \frac{\pi}{2}, \tilde{\phi}, 0\right)\right] .
$$

All fourteen vector structure functions and sixteen of the tensor ones are then determined. For the remaining functions $f_{T T}^{21+}(X), f_{T T}^{21-}(X), f_{T T}^{22+}(X), f_{T T}^{22-}(X)$ and $\left(\rho_{L} f_{L}^{21}(X)+\rho_{T} f_{T}^{21}(X)\right)$ one needs four measurements in order to determine them. The last combination and the one in (D54) requires an additional Rosenbluth separation. A survey on the number of asymmetry measurements needed for the separation of a given structure function is given in Tab. X.

\section{APPENDIX E: EXPLICIT EXPRESSIONS FOR THE MATRIX REPRESENTATION OF $\mathcal{U}_{X}^{\lambda^{\prime} \lambda I M}$}

For the matrix representation of the $\mathcal{U}_{X}^{\lambda^{\prime} \lambda I M}$ in (101) we switch to the spherical representation according to (A8)

$$
\widetilde{C}_{j^{\prime} j}^{I M \lambda^{\prime} \lambda}(X)=\sum_{\tau^{\prime} \nu^{\prime} \tau \nu} s_{\alpha^{\prime}}^{\tau^{\prime} \nu^{\prime}} s_{\alpha}^{\tau \nu} C_{m_{1} m_{2} \lambda_{d}}^{m_{1}^{\prime} m_{2}^{\prime} \lambda_{d}^{\prime}}\left(\tau^{\prime} \nu^{\prime} \tau \nu I M\right)
$$

The explicit forms of the $C_{m_{1} m_{2} \lambda_{d}}^{m_{1}^{\prime} m_{2}^{\prime} \lambda_{d}^{\prime}}\left(\tau^{\prime} \nu^{\prime} \tau \nu I M\right)$ for the helicity, hybrid and standard bases are:

(i) Helicity basis with labeling $\left(\lambda_{p}^{(\prime)}, \lambda_{n}^{(\prime)}, \lambda^{(\prime)}, \lambda_{d}^{(\prime)}\right)$

$$
C_{\lambda_{p} \lambda_{n} \lambda_{d}}^{\lambda_{p}^{\prime} \lambda_{d}^{\prime} \lambda_{d}^{\prime}}\left(\tau^{\prime} \nu^{\prime} \tau \nu I M\right)=2 \sqrt{3}(-)^{\lambda_{p}^{\prime}+\lambda_{n}^{\prime}-\lambda_{d}+\tau^{\prime}+\tau} \hat{\tau}^{\prime} \hat{\tau} \hat{I}\left(\begin{array}{ccc}
1 & 1 & I \\
\lambda_{d}^{\prime} & -\lambda_{d} & M
\end{array}\right)\left(\begin{array}{ccc}
\frac{1}{2} & \frac{1}{2} & \tau^{\prime} \\
\lambda_{p}^{\prime} & -\lambda_{p} & \nu^{\prime}
\end{array}\right)\left(\begin{array}{ccc}
\frac{1}{2} & \frac{1}{2} & \tau \\
\lambda_{n}^{\prime} & -\lambda_{n} & \nu
\end{array}\right)
$$

(ii) Hybrid basis with the labeling $\left(\tilde{\lambda}_{p}^{(\prime)}, \tilde{\lambda}_{n}^{(\prime)}, \lambda^{(\prime)}, \tilde{\lambda}_{d}^{(\prime)}\right)$, where for proton, neutron and deuteron the spin projections refer to the transverse $y$-axis,

$$
C_{\tilde{\lambda}_{p} \tilde{\lambda}_{n} \tilde{\lambda}_{d}}^{\tilde{\lambda}_{\lambda}^{\prime} \tilde{\lambda}_{\lambda}^{\prime} \tilde{\lambda}_{d}^{\prime}}\left(\tau^{\prime} \nu^{\prime} \tau \nu I M\right)=2 \sqrt{3} \omega_{1 \tilde{\lambda}_{d} \tilde{\lambda}_{d}^{\prime}}^{I M}\left(\omega_{\frac{1}{2} \tilde{\lambda}_{p}^{\prime} \tilde{\lambda}_{p}}^{\tau^{\prime} \nu^{\prime}}\right)^{*}\left(\omega_{\frac{1}{2} \tilde{\lambda}_{n}^{\prime} \tilde{\lambda}_{n}}^{\tau \nu}\right)^{*}
$$

where

$$
\omega_{j m^{\prime} m}^{J M}=(-)^{j-m^{\prime}} \hat{J} \sum_{M^{\prime}}\left(\begin{array}{ccc}
j & J & j \\
-m^{\prime} & M^{\prime} & m
\end{array}\right) i^{M^{\prime}-M} d_{M^{\prime} M}^{J}\left(\frac{\pi}{2}\right) .
$$


(iii) Standard basis with index labeling $\left(s^{(\prime)}, m_{s}^{(\prime)}, \lambda^{(\prime)}, m_{d}^{(\prime)}\right)$

$$
\begin{aligned}
C_{s m_{s} \lambda_{d}}^{s^{\prime} m_{s}^{\prime} \lambda_{d}^{\prime}}\left(\tau^{\prime} \nu^{\prime} \tau \nu I M\right)= & 2 \sqrt{3}(-)^{1-\lambda_{d}+s^{\prime}-m_{s}^{\prime}+\tau^{\prime}+\tau} \hat{s} \hat{s}^{\prime} \hat{\tau}^{\prime} \hat{\tau} \hat{I}\left(\begin{array}{ccc}
1 & 1 & 1 \\
\lambda_{d}^{\prime} & -\lambda_{d} & M
\end{array}\right) \\
& \sum_{S \sigma} \hat{S}^{2}\left(\begin{array}{ccc}
\tau^{\prime} & \tau & S \\
\nu^{\prime} & \nu & -\sigma
\end{array}\right)\left(\begin{array}{ccc}
s^{\prime} & s & S \\
m_{s}^{\prime} & -m_{s} & -\sigma
\end{array}\right)\left\{\begin{array}{ccc}
\frac{1}{2} & \frac{1}{2} & \tau^{\prime} \\
\frac{1}{2} & \frac{1}{2} & \tau \\
s^{\prime} & s & S
\end{array}\right\} .
\end{aligned}
$$

\section{APPENDIX F: EXPLICIT EXPRESSIONS OF THE ONE-BODY CURRENT MATRIX IN PLANE WAVE BORN APPROXIMATION IN THE STANDARD REPRESENTATION}

In the plane wave BoRN approximation (PWBA), the final $n p$-scattering state is replaced by a pure plane wave. The deuteron wave function has the form

$$
\left\langle\vec{r} \mid 1 \lambda_{d}\right\rangle=\left\langle r \theta \phi \mid 1 \lambda_{d}\right\rangle=\sum_{l=0,2} \frac{u_{l}(r)}{r}\left\langle\theta \phi \mid(l 1) 1 \lambda_{d}\right\rangle
$$

and the final state plane wave

$$
\left\langle\vec{r} \mid \vec{k} s m_{s}\right\rangle=\frac{e^{i \vec{k} \cdot \vec{r}}}{(2 \pi)^{3 / 2}}\left|s m_{s}\right\rangle
$$

The quantization axis is chosen along $\vec{k}$, the relative $n p$ momentum in the final state.

Taking the current in the DiRAC-PAULI form, one obtains for the one-body current matrix element in the standard representation for the nonrelativistic charge density [32]

$$
\left\langle s m_{s}\left|\rho^{N R}(\vec{q}, \vec{P})\right| 1 \lambda_{d}\right\rangle=\delta_{s 1} \sum_{j=1,2} \sum_{l m_{l}} C_{l m_{l} 1 m_{s}}^{1 \lambda_{d}} F_{1 j}\left\langle\vec{k}_{j} \mid l m_{l}\right\rangle
$$

where $C_{l m_{l} 1 m_{s}}^{1 \lambda_{d}}$ denotes a CLEBSCH-GoRdAn coefficient $\left\langle l m_{l} 1 m_{s} \mid 1 \lambda_{d}\right\rangle$. The leading order relativistic contributions arise from the $p / M$-expansion of the DIRAC current and from the wave function boost. The corresponding charge density operators are denoted by $\rho^{R}$ and $\rho^{B}$, respectively,

$$
\begin{gathered}
\left\langle s m_{s}\left|\rho^{R}(\vec{q}, \vec{P})\right| 1 \lambda_{d}\right\rangle=-\frac{1}{8 M^{2}} \sum_{j=1,2} \sum_{l m_{l} m_{s}^{\prime}} C_{l m_{l} 1 m_{s}^{\prime}}^{1 \lambda_{d}}\left(2 G_{M j}-F_{1 j}\right)\left\{\delta_{s 1} \delta_{m_{s} m_{s}^{\prime}} q^{2}+S_{j, k \times q}^{s m_{s} m_{s}^{\prime}}\right\}\left\langle\vec{k}_{j} \mid l m_{l}\right\rangle, \\
\left\langle s m_{s}\left|\rho^{B}(\vec{q}, \vec{P})\right| 1 \lambda_{d}\right\rangle=\frac{1}{16 M^{2}} \sum_{j=1,2} \sum_{l m_{l} m_{s}^{\prime}} C_{l m_{l} 1 m_{s}^{\prime}}^{1 \lambda_{d}} F_{1 j}\left\{\left(-q^{2} \delta_{s 1} \delta_{m_{s} m_{s}^{\prime}}+S_{1, k \times q}^{s m_{s} m_{s}^{\prime}}+S_{2, k \times q}^{s m_{s} m_{s}^{\prime}}\right)\left\langle\vec{k}_{j} \mid l m_{l}\right\rangle\right. \\
\left.\quad-\left(s_{j} q^{2}-\Pi_{q}\right) \delta_{s 1} \delta_{m_{s} m_{s}^{\prime}}\left\langle\vec{k}_{j}|i \vec{q} \cdot \vec{r}| l m_{l}\right\rangle\right\} .
\end{gathered}
$$

Similarly, one has for the nonrelativistic transverse current $(\lambda= \pm 1)$

$$
\left\langle s m_{s}\left|J_{\lambda}^{N R}(\vec{q}, \vec{P})\right| 1 \lambda_{d}\right\rangle=\frac{e^{i \lambda \phi}}{2 M} \sum_{j=1,2} \sum_{l m_{l} m_{s}^{\prime}} C_{l m_{l} 1 m_{s}^{\prime}}^{1 \lambda_{d}}\left\{F_{1 j} \delta_{s 1} \delta_{m_{s} m_{s}^{\prime}} \Pi_{j, \lambda}+G_{M j} S_{j, q, \lambda}^{s m_{s} m_{s}^{\prime}}\right\}\left\langle\vec{k}_{j} \mid l m_{l}\right\rangle .
$$

For the leading order relativistic current contributions we again distinguish $J_{\lambda}^{R}$ from expanding the DiRAC current 
and two contributions from the boost, $J_{c, \lambda}^{B}$ and $J_{s, \lambda}^{B}$, with respect to the nonrelativistic convection and spin current,

$$
\begin{aligned}
& \left\langle s m_{s}\left|J_{\lambda}^{R}(\vec{q}, \vec{P})\right| 1 \lambda_{d}\right\rangle=-\frac{e^{i \lambda \phi}}{16 M^{3}} \sum_{j=1,2} \sum_{l m_{l} m_{s}^{\prime}} C_{l m_{l} 1 m_{s}^{\prime}}^{1 \lambda_{d}}\left\{\Pi_{j, \lambda}\left[\left(F_{1 j} k_{j}^{2}+G_{M j} q^{2}\right) \delta_{s 1} \delta_{m_{s} m_{s}^{\prime}}+\left(G_{M j}-F_{1 j}\right) S_{j, k \times q}^{s m_{s} m_{s}^{\prime}}\right]\right. \\
& \left.+G_{M j}\left[\left(k_{j}^{2}+q^{2}\right) S_{j, q, \lambda}^{s m_{s} m_{s}^{\prime}}+\Pi_{j, q} S_{j, \pi, \lambda}^{s m_{s} m_{s}^{\prime}}\right]\right\}\left\langle\vec{k}_{j} \mid l m_{l}\right\rangle, \\
& \left\langle s m_{s}\left|J_{c, \lambda}^{B}(\vec{q}, \vec{P})\right| 1 \lambda_{d}\right\rangle=\frac{e^{i \lambda \phi}}{32 M^{3}} \sum_{j=1,2} \sum_{l m_{l} m_{s}^{\prime}} C_{l m_{l} 1 m_{s}^{\prime}}^{1 \lambda_{d}} F_{1 j} \Pi_{j, \lambda}\left\{\left(-q^{2} \delta_{s 1} \delta_{m_{s} m_{s}^{\prime}}+S_{1, k \times q}^{s m_{s} m_{s}^{\prime}}+S_{2, k \times q}^{s m_{s} m_{s}^{\prime}}\right)\left\langle\vec{k}_{j} \mid l m_{l}\right\rangle\right. \\
& \left.-\left(s_{j} q^{2}-\Pi_{q}\right) \delta_{s 1} \delta_{m_{s} m_{s}^{\prime}}\left\langle\vec{k}_{j}|i \vec{q} \cdot \vec{r}| l m_{l}\right\rangle\right\}, \\
& \left\langle s m_{s}\left|J_{s, \lambda}^{B}(\vec{q}, \vec{P})\right| 1 \lambda_{d}\right\rangle=\frac{e^{i \lambda \phi}}{32 M^{3}} \sum_{j=1,2} \sum_{l m_{l} m_{s}^{\prime}} C_{l m_{l} 1 m_{s}^{\prime}}^{1 \lambda_{d}} G_{M j}\left\{-\left(s_{j} q^{2}-\Pi_{q}\right) S_{j, q, \lambda}^{s m_{s} m_{s}^{\prime}}\left\langle\vec{k}_{j}|i \vec{q} \cdot \vec{r}| l m_{l}\right\rangle\right. \\
& \left.+\left[-\left(\left(q^{2}-s_{j} \Pi_{q}\right) S_{j, q, \lambda}^{s m_{s} m_{s}^{\prime}}+q^{2} S_{j, k, \lambda}^{s m_{s} m_{s}^{\prime}}\right)+q^{2} \Pi_{j, \lambda} \delta_{s 1} \delta_{m_{s} m_{s}^{\prime}}+2 \lambda k q^{2} \sum_{j^{\prime} \neq j} s_{j^{\prime}} S_{j, j^{\prime}}^{s m_{s} m_{s}^{\prime}}\right]\left\langle\vec{k}_{j} \mid l m_{l}\right\rangle\right\},
\end{aligned}
$$

where $\vec{k}_{j}=\vec{k}-s_{j} \vec{q} / 2$ with $s_{j}= \pm 1$ for $j=1$ and 2 , respectively. The FouRIER transform of the orbital components of the deuteron wave function is given by

$$
\begin{aligned}
\left\langle\vec{p} \mid l m_{l}\right\rangle & =\int d^{3} r e^{-i \vec{p} \cdot \vec{r}}\left\langle\vec{r} \mid l m_{l}\right\rangle \\
& =(-)^{l / 2} 4 \pi Y_{l m_{l}}(\hat{p}) \int_{0}^{\infty} \operatorname{drru}_{l}(r) j_{l}(p r),
\end{aligned}
$$

and

$$
\left\langle\vec{p}|i \vec{q} \cdot \vec{r}| l m_{l}\right\rangle=-\vec{q} \cdot \nabla_{p}\left\langle\vec{p} \mid l m_{l}\right\rangle .
$$

For convenience the various spin matrix elements have been denoted by

$$
\begin{aligned}
& S_{1 j}^{s m_{s} m_{s}^{\prime}}=\left\langle\left(\frac{1}{2} \frac{1}{2}\right) s m_{s}\left|\sigma_{j, m_{s}-m_{s}^{\prime}}\right|\left(\frac{1}{2} \frac{1}{2}\right) 1 m_{s}^{\prime}\right\rangle=(-1)^{s-m_{s}}\left(\begin{array}{ccc}
s & 1 & 1 \\
-m_{s} & m_{s}-m_{s}^{\prime} & m_{s}^{\prime}
\end{array}\right)\left\langle s\left\|\sigma_{j}^{[1]}\right\| 1\right\rangle, \\
& S_{j, k \times q}^{s m_{s} m_{s}^{\prime}}=e^{-i \lambda \phi}\left\langle\left(\frac{1}{2} \frac{1}{2}\right) s m_{s}\left|2 s_{j} i \vec{\sigma}_{j} \cdot(\vec{k} \times \vec{q})\right|\left(\frac{1}{2} \frac{1}{2}\right) 1 m_{s}^{\prime}\right\rangle=2 s_{j} k q\left(m_{s}-m_{s}^{\prime}\right) S_{1 j}^{s m_{s} m_{s}^{\prime}} d_{0, m_{s}-m_{s}^{\prime}}^{1}(\theta) \text {, } \\
& S_{j, q, \lambda}^{s m_{s} m_{s}^{\prime}}=e^{-i \lambda \phi}\left\langle\left(\frac{1}{2} \frac{1}{2}\right) s m_{s}\left|i\left(\vec{\sigma}_{j} \times \vec{q}\right)_{\lambda}\right|\left(\frac{1}{2} \frac{1}{2}\right) 1 m_{s}^{\prime}\right\rangle=\lambda q S_{1 j}^{s m_{s} m_{s}^{\prime}} d_{\lambda, m_{s}-m_{s}^{\prime}}^{1}(\theta), \\
& S_{j, k, \lambda}^{s m_{s} m_{s}^{\prime}}=e^{-i \lambda \phi}\left\langle\left(\frac{1}{2} \frac{1}{2}\right) s m_{s}\left|2 s_{j} i\left(\vec{\sigma}_{j} \times \vec{k}\right)_{\lambda}\right|\left(\frac{1}{2} \frac{1}{2}\right) 1 m_{s}^{\prime}\right\rangle=2 s_{j} k\left(m_{s}-m_{s}^{\prime}\right) S_{1 j}^{s m_{s} m_{s}^{\prime}} d_{\lambda, m_{s}-m_{s}^{\prime}}^{1}(\theta) \text {, } \\
& S_{j, \pi, \lambda}^{s m_{s} m_{s}^{\prime}}=e^{-i \lambda \phi}\left\langle\left(\frac{1}{2} \frac{1}{2}\right) s m_{s}\left|i\left(\vec{\sigma}_{j} \times\left(2 s_{j} \vec{k}-\vec{q}\right)\right)_{\lambda}\right|\left(\frac{1}{2} \frac{1}{2}\right) 1 m_{s}^{\prime}\right\rangle=S_{j, k, \lambda}^{s m_{s} m_{s}^{\prime}}-S_{j, q, \lambda}^{s m_{s} m_{s}^{\prime}}, \\
& S_{j, j^{\prime}}^{s m_{s} m_{s}^{\prime}}=(-)^{s-m_{s}} \sum_{s^{\prime \prime} m_{s}^{\prime \prime}}\left(m_{s}^{\prime \prime}-m_{s}^{\prime}\right)\left(\begin{array}{ccc}
s & 1 & s^{\prime \prime} \\
-m_{s} & m_{s}-m_{s}^{\prime \prime} & m_{s}^{\prime \prime}
\end{array}\right) \\
& d_{\lambda, m_{s}-m_{s}^{\prime \prime}}^{1}(\theta) d_{0, m_{s}^{\prime \prime}-m_{s}^{\prime}}^{1}(\theta) S_{1 j^{\prime}}^{s^{\prime \prime} m_{s}^{\prime \prime} m_{s}^{\prime}}\left\langle s\left\|\sigma_{j}^{[1]}\right\| s^{\prime \prime}\right\rangle .
\end{aligned}
$$

Furthermore,

$$
\begin{aligned}
\Pi_{q}(\theta) & =2 k q \cos \theta, \\
\Pi_{j, q}(\theta) & =s_{j} \Pi_{q}-q^{2}, \\
\Pi_{j, \lambda}(\theta) & =2 s_{j} k d_{\lambda, 0}^{1}(\theta) .
\end{aligned}
$$

Taking the current in the SACHS form, the expressions for the nonrelativistic and boost currents are simply obtained 
from (F3), (F5), (F6), (F8) and (F9) by substituting $F_{1 j}$ by $G_{E j}$. For the other relativistic contributions one finds

$$
\begin{aligned}
\left\langle s m_{s}\left|\rho^{R}(\vec{q}, \vec{P})\right| 1 \lambda_{d}\right\rangle= & \left.-\frac{1}{8 M^{2}} \sum_{j=1,2} \sum_{l m_{l} m_{s}^{\prime}} C_{l m_{l} 1 m_{s}^{\prime}}^{1 \lambda_{d}}\left\{G_{E j} \delta_{s 1} \delta_{m_{s} m_{s}^{\prime}} q^{2}+\left(2 G_{M j}-G_{E j}\right) S_{j, k \times q}^{s m_{s} m_{s}^{\prime}}\right)\right\}\left\langle\vec{k}_{j} \mid l m_{l}\right\rangle, \\
\left\langle s m_{s}\left|J_{\lambda}^{R}(\vec{q}, \vec{P})\right| 1 \lambda_{d}\right\rangle= & -\frac{e^{i \lambda \phi}}{16 M^{3}} \sum_{j=1,2} \sum_{l m_{l} m_{s}^{\prime}} C_{l m_{l} 1 m_{s}^{\prime}}^{1 \lambda_{d}}\left\{G_{M j}\left[\left(k_{j}^{2}+q^{2}\right) S_{j, q, \lambda}^{s m_{s} m_{s}^{\prime}}+\Pi_{j, q} S_{j, \pi, \lambda}^{s m_{s} m_{s}^{\prime}}\right]\right. \\
& \left.+\Pi_{j, \lambda}\left[\left(G_{E j}\left(2 q^{2}+k_{j}^{2}\right)-G_{M j} q^{2}\right) \delta_{s 1} \delta_{m_{s} m_{s}^{\prime}}-\left(G_{E j}-G_{M j}\right) S_{j, k \times q}^{s m_{s} m_{s}^{\prime}}\right]\right\}\left\langle\vec{k}_{j} \mid l m_{l}\right\rangle .
\end{aligned}
$$

[1] W. Leidemann, E.L. Tomusiak, H. Arenhövel, Phys. Rev. C 43, 1022 (1991).

[2] H. Arenhövel, W. Leidemann, E.L. Tomusiak, Phys. Rev. C 46, 455 (1992).

[3] H. Arenhövel, W. Leidemann, E.L. Tomusiak, Few-Body Syst. 15, 109 (1993).

[4] H. Arenhövel, W. Leidemann, E.L. Tomusiak, Phys. Rev. C 52, 1232 (1995).

[5] H. Arenhövel, W. Leidemann, E.L. Tomusiak, Nucl. Phys. 641, 517 (1998).

[6] H. Arenhövel, W. Leidemann, E.L. Tomusiak, Few-Body Syst. 28, 147 (2000).

[7] H. Arenhövel, Few-Body Syst. 4, 55 (1988).

[8] H. Arenhövel and K.-M. Schmitt, Few-Body Syst. 8, 77 (1990).

[9] V. Dmitrasinovic and F. Gross, Phys. Rev. C 40, 2479 (1989)

[10] H. Arenhövel, W. Leidemann, E.L. Tomusiak, Eur. Phys. J. A 14, 491 (2002).

[11] F.R. Halpern, Special Relativity and Quantum Mechanics. Englewood Cliffs, Prentice-Hall 1968

[12] M.A. Martin and T.D. Spearman, Elementary Particle Theory. Amsterdam, North-Holland 1970

[13] D.R. Giebink, Phys. Rev. C 32, 502 (1985)

[14] T.W. Donnelly and A.S. Raskin, Ann. Phys. (N.Y.) 169, 247 (1986).

[15] E.M. Rose, Elementary Theory of Angular Momentum, Wiley New York 1957.

[16] B.A. Robson, The Theory of Polarization Phenomena. Oxford, Clarendon Press 1974

[17] F. Partovi, Ann. Phys. (N.Y.) 27, 79 (1964).

[18] H. Arenhövel and M. Sanzone, Few-Body Syst. Suppl. 3, 1 (1991).

[19] M. Kawaguchi, Phys. Rev. 111, 1314 (1958).

[20] A. Cambi and B. Mosconi, Phys. Rev. C 26, 2358 (1982).

[21] A.S. Raskin and T.W. Donnelly, Ann. Phys. (N.Y.) 191, 78 (1989).

[22] J.M. Blatt and L.C. Biedenharn, Phys. Rev. 86, 399 (1952).

[23] W. Fabian and H. Arenhövel, Nucl. Phys. A 314, 253 (1979).

[24] H. Arenhövel, M. Danos, and H.T. Williams, Nucl. Phys. A 314

[25] W. Leidemann and H. Arenhövel, Nucl. Phys. A 465, 573 (1987).

[26] F. Ritz, H. Göller, T. Wilbois, and H. Arenhövel, Phys. Rev. C 55, 2214 (1997).

[27] R. Machleidt, K. Holinde, and Ch. Elster, Phys. Rep. 149, 1 (1987).

[28] M.M. Nagels, T.A. Rijken, and J.J. de Swart, Phys. Rev. D 17, 768 (1978).

[29] M. Lacombe, B. Loiseau, J.M. Richard, R. Vinh Mau, J. Côté, P. Pirès, and R. de Tourreil, Phys. Rev. C 21, 861 (1980).

[30] R.W. Wiringa, R. Smith, and T.L. Ainsworth, Phys. Rev. C 29, 1207 (1984).

[31] R.W. Wiringa, V.G.J. Stoks, and R. Schiavilla, Phys. Rev. C 51, 38 (1995).

[32] G. Beck and H. Arenhövel, Few-Body Syst. 13, 165 (1992); 15, 37 (1993) (E).

[33] S. Galster et al., Nucl. Phys. B 32, 221 (1971).

[34] S. Blatnik, N. Zovko, Acta Phys. Austriaca 39, 62 (1974).

[35] G. Höhler et al., Nucl. Phys. B 114, 505 (1976).

[36] M.F. Gari, W. Krümpelmann, Phys. Lett. B 274, 159 (1992).

[37] H.W. Hammer, U.-G. Meißner, and D. Drechsel, Phys. Lett. B 385, 343 (1996).

[38] H. Arenhövel, Phys. Lett. B 199, 13 (1987).

[39] E.L. Tomusiak and H. Arenhövel, Phys. Lett. B 206, 187 (1988).

[40] H. Arenhövel, W. Leidemann, E.L. Tomusiak, Z. Phys. A 331, 123 (1988); A 334, 363 (1989) [Erratum].

[41] M. Schwamb and H. Arenhövel, Nucl. Phys. A 696, 556 (2001).

[42] D.O. Riska, Phys. Scrip. 31, 471 (1985).

[43] A. Buchmann, W. Leidemann, and H. Arenhövel, Nucl. Phys. A 443, 726 (1985).

[44] H. Arenhövel and M. Schwamb, Eur. Phys. J. A 12, 207 (2001).

[45] H. Arenhövel, Z. Phys. A 302, 25 (1981).

[46] M. Gari and H. Hebach, Phys. Rep. 72, 1 (1981).

[47] H.J. Weber and H. Arenhövel, Phys. Rep. 36, 279 (1978).

[48] A.M. Green and M. Sainio, J. Phys. G 8, 1337 (1982).

[49] W. Leidemann, diploma thesis, Mainz 1980. 
[50] J.L. Friar, Ann. Phys. (NY) 104, 180 (1977).

[51] E. Truhlik and J. Adam, Jr., Nucl. Phys. A 492, 529 (1989).

[52] H. Göller and H. Arenhövel, Few-Body Syst. 13, 117 (1992).

[53] K. Tamura, T. Niwa, T. Sato, and H. Ohtsubo, Nucl. Phys. A 536, 597 (1992).

[54] J. Adam, Jr. and H. Arenhövel, Nucl. Phys. A 614, 289 (1997).

[55] T. Wilbois, G. Beck, and H. Arenhövel, Few-Body Syst. 15, 39 (1993).

[56] S. Auffret et al., Phys. Rev. Lett. 55, 1362 (1985).

[57] R.G. Arnold et al., Phys. Rev. C 42, R1 (1990).

[58] G.G. Simon et al., Nucl. Phys. A 324, 277 (1979).

[59] B.P. Quinn et al., Phys. Rev. C 37, 1609 (1988).

[60] B. Boden et al., Nucl. Phys. A 549, 471 (1992).

[61] H.J. Bulten et al., Phys. Rev. Lett. 74, 4775 (1995).

[62] A. Pellegrino et al., Phys. Rev. Lett. 78, 4011 (1997).

[63] K.I. Blomqvist et al., Phys. Lett. B 424, 33 (1998).

[64] W.-J. Kasdorp et al., Few-Body Syst. 25, 115 (1998).

[65] S.M. Dolfini et al., Phys. Rev. C 60, 064622 (1999).

[66] M. Ostrick et al., Phys. Rev. Lett. 83, 276 (1999).

[67] D.M. Nikolenko et al., Nucl. Phys. A 684, 525c (2001).

[68] H. Zhu et al., Phys. Rev. Lett. 87, 081801 (2001).

[69] Z.-L. Zhou et al., Phys. Rev. Lett. 87, 172301 (2001).

[70] P. von Neuman-Cosel et al., Phys. Rev. Lett. 88, 202304 (2002).

[71] P.E. Ulmer et al., Phys. Rev. Lett. 89, 062301 (2002).

[72] U. Müller et al., Proc. Int. School on Nuclear Physics, Erice, Italy, Prog. Part. Nucl. Phys. 50, 483 (2003).

[73] D.M. Nikolenko et al., Phys. Rev. Lett. 90, 072501 (2003).

[74] R. Madey et al., Eur. Phys. J. A 17, 323 (2003).

[75] R. Madey et al., Phys. Rev. Lett. 91, 122002 (2003). 